\title{
Magnetic quivers from brane webs with 05 planes
}

\author{
Antoine Bourget, ${ }^{a}$ Julius F. Grimminger, ${ }^{a}$ Amihay Hanany, ${ }^{a}$ Marcus Sperling ${ }^{b}$ \\ and Zhenghao Zhong $^{a}$ \\ ${ }^{a}$ Theoretical Physics Group, The Blackett Laboratory, Imperial College London, \\ Prince Consort Road, London, SWY 2AZ, U.K. \\ ${ }^{b}$ Yau Mathematical Sciences Center, Tsinghua University, \\ Haidian District, Beijing, 100084, China \\ E-mail: a.bourget@imperial.ac.uk, julius.grimminger17@imperial.ac.uk, \\ a.hanany@imperial.ac.uk, marcus.sperling@univie.ac.at, \\ zhenghao.zhong14@imperial.ac.uk
}

ABSTRACT: Magnetic quivers have led to significant progress in the understanding of gauge theories with 8 supercharges at UV fixed points. For a given low-energy gauge theory realised via a Type II brane construction, there exist magnetic quivers for the Higgs branches at finite and infinite gauge coupling. Comparing these moduli spaces allows one to study the non-perturbative effects when transitioning to the fixed point. For $5 \mathrm{~d} \mathcal{N}=1 \mathrm{SQCD}$, 5 -brane webs have been an important tool for deriving magnetic quivers. In this work, the emphasis is placed on 5-brane webs with orientifold 5-planes which give rise to $5 \mathrm{~d}$ theories with orthogonal or symplectic gauge groups. For this set-up, the magnetic quiver prescription is derived and contrasted against a unitary magnetic quiver description extracted from an $\mathrm{O}^{-}$construction. Further validation is achieved by a derivation of the associated Hasse diagrams. An important class of families considered are the orthogonal exceptional $E_{n}$ families $(-\infty<n \leq 8)$, realised as infinite coupling Higgs branches of $\operatorname{Sp}(k)$ gauge theories with fundamental matter. In particular, the moduli spaces are realised by a novel type of magnetic quivers, called unitary-orthosymplectic quivers.

Keywords: Brane Dynamics in Gauge Theories, D-branes, Extended Supersymmetry, Supersymmetric Gauge Theory

ArXiv EPrint: 2004.04082 


\section{Contents}

1 Introduction 1

2 Magnetic quivers and known examples $\quad 7$

$\begin{array}{lll}2.1 \text { Set-up } & 7\end{array}$

2.2 Magnetic quivers 8

$\begin{array}{ll}2.3 \text { Single } \mathrm{Sp}(1) \text { gauge group } & 10\end{array}$

$\begin{array}{lll}2.3 .1 & E_{8}: \operatorname{Sp}(1) \text { with } 7 \text { flavours } & 10\end{array}$

$\begin{array}{lll}\text { 2.3.2 } & E_{7}: \operatorname{Sp}(1) \text { with } 6 \text { flavours } & 14\end{array}$

$\begin{array}{lll}\text { 2.3.3 } & E_{6}: \operatorname{Sp}(1) \text { with } 5 \text { flavours } & 17\end{array}$

$\begin{array}{lll}2.3 .4 & E_{5}: \operatorname{Sp}(1) \text { with } 4 \text { flavours } & 19\end{array}$

2.3.5 $E_{4}: \operatorname{Sp}(1)$ with 3 flavours $\quad 21$

2.3.6 $E_{3}: \operatorname{Sp}(1)$ with 2 flavours 24

2.3.7 $E_{2}: \operatorname{Sp}(1)$ with 1 flavour $\quad 25$

2.3.8 $E_{1}$ : pure $\operatorname{Sp}(1)$ theory 27

$2.3 .9 E_{0}$ theory 28

2.4 Rules for magnetic quivers 28

3 Single $\mathrm{Sp}(\mathrm{k})$ gauge group $\quad 29$

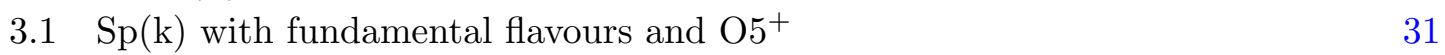

3.1.1 Finite coupling 32

$\begin{array}{lll}3.1 .2 & \text { Infinite coupling } & 35\end{array}$

$\begin{array}{lll}3.2 & \mathrm{Sp}(\mathrm{k}) \text { with fundamental flavours and } \widetilde{\mathrm{O}}^{+} & 37\end{array}$

4 Comparison with $\mathrm{O7}^{-}$construction $\quad 38$

$\begin{array}{lll}4.1 & \text { Finite coupling } & 40\end{array}$

$\begin{array}{lll}4.2 & \text { Infinite coupling } & 42\end{array}$

$\begin{array}{lll}4.3 & \text { Duality with 5d SQCD } & 47\end{array}$

$\begin{array}{ll}4.4 \text { Two realisations } & 47\end{array}$

$\begin{array}{ll}4.5 & \text { Problem of two cones }\end{array}$

5 Hasse diagrams and quiver subtraction $\quad 49$

$\begin{array}{lll}5.1 & E_{8} \text { family } & 49\end{array}$

$\begin{array}{lll}5.2 & E_{7} \text { family } & 50\end{array}$

$\begin{array}{lll}5.3 & E_{6} \text { family } & 52\end{array}$

$\begin{array}{lll}5.4 & E_{5} \text { family } & 53\end{array}$

$\begin{array}{lll}5.5 & E_{4} \text { family } & 54\end{array}$

$\begin{array}{lll}5.6 & E_{3} \text { family } & 55\end{array}$

$\begin{array}{lll}5.7 & E_{2} \text { family } & 56\end{array}$

$5.8 E_{1}$ family $\quad 57$

$5.9 E_{\leq 0}$ families $\quad 58$ 
$\begin{array}{ll}\text { A Background material } & \mathbf{7 0}\end{array}$

A.1 Type II brane configurations with 8 supercharges $\quad 70$

$\begin{array}{lll}\text { A.2 } & \text { 5-branes webs with 7-branes and orientifold 5-planes } & 71\end{array}$

A.3 $3 \mathrm{~d} \mathcal{N}=4$ Coulomb branches $\quad 73$

$\begin{array}{lll}\text { A.4 Summary companion paper } & 74\end{array}$

\section{Introduction}

5-dimensional $\mathcal{N}=1$ gauge theories are perturbatively non-renormalisable and can only meaningfully be defined as mass deformations of renormalisation group fixed points. Initially, these theories have been studied from various aspects: field theory [1-3], brane constructions [4-6], and geometry via M-theory backgrounds with Calabi-Yau singularities [7].

In this work, focus is placed on 5-brane webs in Type IIB superstring theory [4-6] and generalisations that include orientifold planes [8-11]. One advantage of these brane constructions is that they capture the dynamics of the corresponding $5 \mathrm{~d}$ gauge theories and, simultaneously, their UV fixed points. Recent developments using brane webs include [12-26]. As known from the $\mathrm{SU}(2)$ example with $N_{f}<8$ flavours [1,2], it is important to understand the enhancement of the global symmetry of these theories at the fixed point. Hence, this question has been studied via various techniques: for instance, superconformal indices [13, 15, 27-36], Nekrasov partition functions and topological string partition functions $[12,37-44]$. The enhancement has been argued to be due to instanton operators [45-47], which create instanton particles in the UV superconformal field theory. Recall that in 5 dimensions, the instanton is a particle charged under the $\mathrm{U}(1)_{I}$ topological symmetry associated to the conserved current $\operatorname{Tr} *(F \wedge F)$.

Recently, there have been many works devoted to uncover further features [48-51]; in particular, classifications of $5 \mathrm{~d}$ SCFTs [52-54] and $5 \mathrm{~d} \mathcal{N}=1$ gauge theories [55] have been proposed.

An interesting question concerns the Higgs branch of the full vacuum moduli space: for the low-energy effective theory the Higgs branch is described by the hyper-Kähler quotient construction [56]; in contrast, for the Higgs branch $\mathcal{H}_{\infty}$ at the fixed point the same is not true. The first studies $[57,58]$ of $\mathcal{H}_{\infty}$ indicated that in order to capture the geometric features of the moduli space at infinite gauge coupling, $3 \mathrm{~d} \mathcal{N}=4$ Coulomb branches of certain quiver gauge theories are useful. This idea has been further developed and systematised in [24]: for a given 5-brane web, where each external 5-brane ends on a 7-brane, magnetic quivers can be derived such that the $3 \mathrm{~d} \mathcal{N}=4$ Coulomb branches thereof are equivalent geometric descriptions for the finite and infinite coupling $5 \mathrm{~d} \mathcal{N}=1$ Higgs branches. 
So far, the magnetic quiver description has only been available for $5 \mathrm{~d}$ gauge theories with special unitary gauge groups. However, many interesting $5 \mathrm{~d}$ dualities, see for instance $[16,18,53,55]$, are between unitary and orthogonal or symplectic gauge groups. Hence, it is natural to further develop the understanding of Higgs branches of $5 \mathrm{~d}$ theories with orthogonal or symplectic groups. Suitable 5-brane web realisations either contain O5 or O7 planes. In this work, the focus is placed on 5-brane webs in the presence of $\mathrm{O} 5$ orientifolds. For single gauge groups with fundamental matter, the field theory classification of theories with non-trivial interacting fixed point has been presented in [3]; the subsequent brane construction [8] confirmed these results. However, more non-trivial fixed points have been proposed in [9].

Consider the Higgs branch moduli space in more detail. To begin with, the finite coupling, or classical, Higgs branches are conventionally treated by an F and D-term analysis. However, it is a known fact that $\operatorname{Sp}(k)$ theories with $N_{f}<2 k$ fundamental flavours and $\mathrm{O}(k)$ theories with $N_{f} \leq k-3$ fundamental flavours do not admit complete Higgsing. Consequently, their analysis is currently incomplete. For instance, the analogous behaviour exists for $5 \mathrm{~d} \mathcal{N}=1 \mathrm{SU}(k) \mathrm{SQCD}$ with $N_{f}<2 k$, which has only recently been addressed in [59]. In terms of brane webs, the quaternionic Higgs branch degrees of freedom can be counted by a decomposition into independent subwebs, as introduced in [5] and demonstrated for 5-brane webs with O5 planes in [10]. Moving on to the infinite coupling Higgs branches, the enhancement of the global symmetry has been studied via field theory [35] and brane webs [9]. Moreover, the counting of additional new Higgs branch dimension at the fixed point has been demonstrated in [10]. Hence, dimension and global symmetry of $\mathcal{H}_{\infty}$ are known, but no geometrical description has been provided yet.

This is precisely the first aim of the present paper: to provide an improved description of finite and infinite coupling Higgs branches. The approach taken is known as magnetic quivers [24, 60,61] (see also [62]): in brief, a magnetic quiver $\mathrm{Q}$ is a combinatorial object that is derived from the Type II brane configuration with 8 supercharges, describing a given theory $T$ in a certain phase $\mathcal{P}$. The Higgs branch of $T$ in that phase equals the $3 \mathrm{~d} \mathcal{N}=4$ Coulomb branch of the magnetic quiver, meaning that the combinatorial data is taken as an input to derive a space of dressed monopole operators in the sense of [63]. Thus,

$$
\mathcal{H}(\text { phase } \mathcal{P} \text { of theory } \mathrm{T})=\mathcal{C}(\text { magnetic quiver } \mathrm{Q}(\mathcal{P}))
$$

holds as equality of moduli spaces. To be more precise: the magnetic quivers compute a geometric space, called Higgs variety. The Higgs branch chiral ring may contain nilpotent operators which makes the full Higgs branch a so called non-reduced scheme, called Higgs Scheme. This problem is addressed for classical $4 \mathrm{~d} \mathcal{N}=2$ SQCD in [59]. In the rest of the paper only the geometric parts of the moduli space are studied and the analysis of nilpotent elements is left for future work. The concept of magnetic quivers has proven itself useful in a variety of cases: for $6 \mathrm{~d} \mathcal{N}=(1,0)$ theories [60,61, 64-66], $5 \mathrm{~d} \mathcal{N}=1$ gauge theories $[24,57,58]$, and 4d Argyres-Douglas theories [67].

The second aim of the present paper is to derive the Hasse diagrams for the finite and infinite coupling Higgs branches. Hasse diagrams for nilpotent orbits were studied in detail by Kraft and Procesi in [68, 69]. As described in [70], the Hasse diagram details the singularity structure of a Higgs branch understood as a symplectic singularity [71]. 
For finite coupling Higgs branches, the Hasse diagram could, for example, be derived from the Higgs mechanism; however, the same method is not applicable at the fixed point. Alternatively, the Hasse diagram can also be derived from (i) the brane web via KraftProcesi transitions [72, 73], or (ii) from quiver subtraction [74] on the magnetic quivers.

Once the combinatorial data of the magnetic quiver is known, the techniques developed allow to extract the following information:

(i) Dimension.

(ii) Global symmetry.

(iii) Representation content of the chiral ring via the Hilbert series.

(iv) Hasse diagram.

(v) Comparison of dimensions, global symmetry, chiral ring representation content, and Hasse diagram between finite and infinite gauge coupling.

Complementing these facts by approaches that rely on the same combinatorial input data, like for instance [75-77], one reaches the conclusion that the entire moduli space geometry is determined by this data.

Summary of main results. The methods developed in this paper yield a multitude of results and are summarised here for convenience.

- Among the most interesting results are the magnetic quivers for $\operatorname{Sp}(k)$ theories with $0 \leq N_{f} \leq 2 k+5$ fundamental flavours, summarised in table 1. Here, two different constructions, by $\mathrm{O}^{+}$and $\mathrm{O}^{-}$orientifolds, give rise to two different sets of magnetic quivers: unitary-orthosymplectic quivers and unitary quivers, respectively. The results are remarkable for various reasons: for the unitary magnetic quivers the global symmetry is straightforwardly evaluated, see tables 2,3 , and, for $k=1$, reproduce the known enhancement for $E_{N_{f}+1}$. Therefore, the cases $\operatorname{Sp}(k)$ with $N_{f}$ fundamentals are referred to as generalised exceptional families. For the unitary-orthosymplectic magnetic quivers, the global symmetry is less obvious from the quiver itself. However, a Hilbert series computation confirms that for each case both magnetic quivers have the same highest weight generating (HWG) function, see table 4 , which is a necessary condition that both quivers describe the same moduli space. The details of the computational challenges are a subject of a companion paper [80].

- Besides the two different magnetic quiver descriptions, the geometry of the Higgs branches is further detailed by the Hasse diagram. A non-trivial consistency check has been passed by verifying that both, the unitary and the unitary-orthosymplectic, magnetic quivers for the exceptional families lead to the same Hasse diagram, see tables 2 and 3. The appearance of minimal nilpotent orbit closures $e_{n}$ of exceptional Lie algebras $E_{n}$ at the top of the infinite coupling Hasse diagram yields another reason for calling these families exceptional families.

While the Hasse diagram derivation for the unitary quivers is straightforward from the results of $[70,74]$, the algorithm for orthosymplectic quivers is more subtle. Quiver subtraction with (framed) orthosymplectic quivers have been used in [65, 81] and the first Hasse diagrams derived in this class have been presented in [61], in the context of $6 \mathrm{~d}$ theories. 


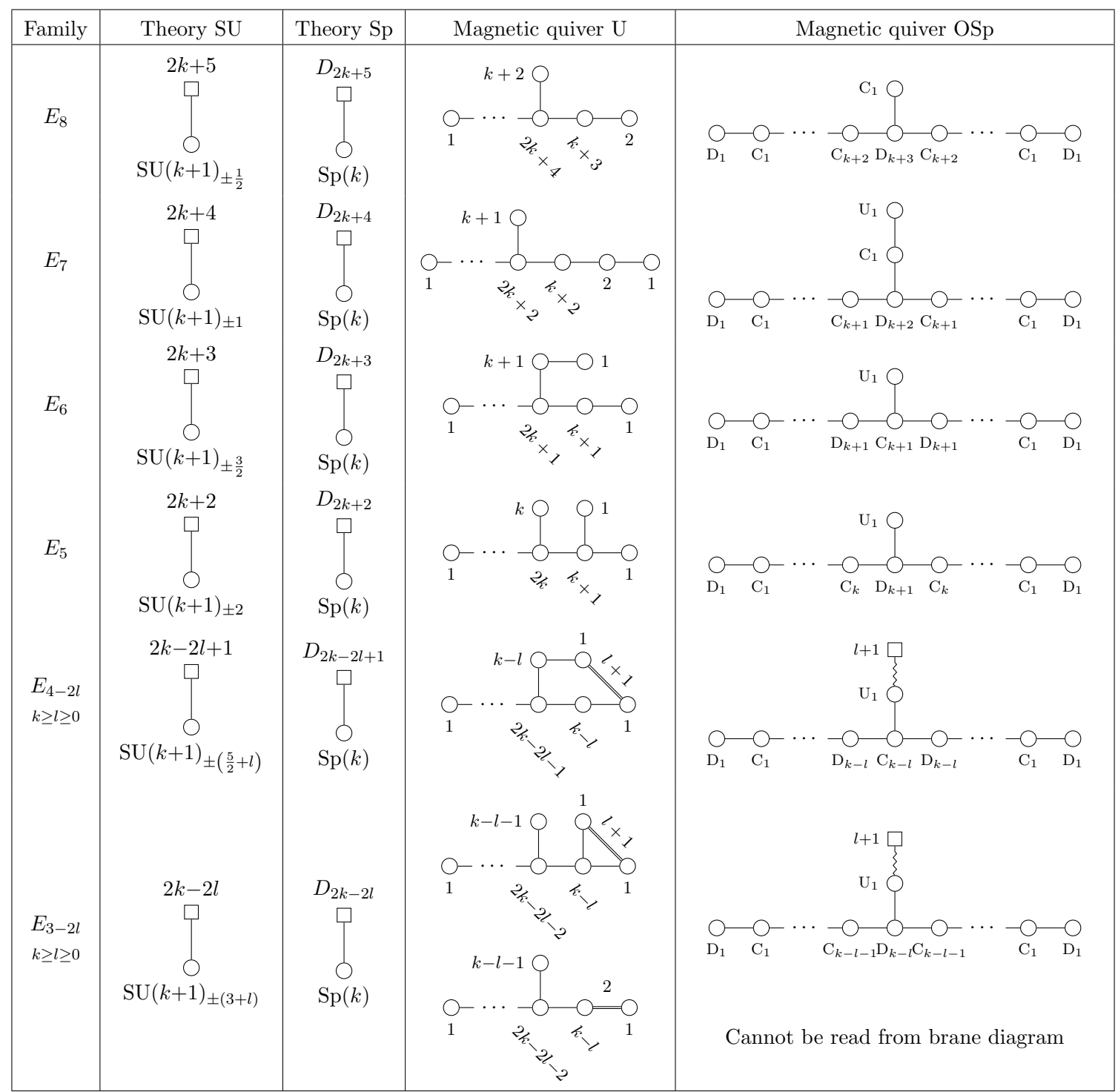

Table 1. Magnetic quivers at infinite coupling. The $5 \mathrm{~d} \mathcal{N}=1$ duality between "Theory SU" and "Theory Sp" has been observed in [16], also [18]. The wiggly link denotes a charge 2 hypermultiplet. The "Magnetic quiver OSp" are derived in section 3, while "Magnetic Quiver U" are subject of section 4 . For $k=0$, the moduli spaces are free hypermultiplets transforming as spinors of the global symmetry. The "Magnetic quiver OSp" for $E_{8,7,6}$ can be obtained from class $\mathrm{S}[78,79]$.

- Concerning orthogonal or symplectic gauge theories without complete Higgsing, the magnetic quivers provide predictions on the finite and infinite coupling Higgs branches.

Outline. The remainder of this paper is organised as follows: after reviewing the set-up, section 2 is devoted to a study of the Higgs branches for $\operatorname{Sp}(1)$ with $N_{f}<8$ flavours. In each case, the brane web and the Higgs branch degrees of freedom are detailed. Building on that, the rules to read off the magnetic quivers are established. Thereafter, the magnetic quiver proposal is applied to $5 \mathrm{~d} \operatorname{Sp}(k)$ theories with fundamental matter in section 3 ; in each case, the 5 -brane webs are detailed and the magnetic quivers are derived. The results 


\begin{tabular}{|c|c|c|c|c|}
\hline Family & $\begin{array}{c}\text { Dimension and } \\
\text { symmetry for } k>1 \\
\text { at finite coupling }\end{array}$ & $\begin{array}{c}\text { Dimension and } \\
\text { symmetry for } k>1 \\
\text { at infinite coupling }\end{array}$ & $\begin{array}{l}\text { Hasse diagram } \\
\text { finite coupling }\end{array}$ & $\begin{array}{l}\text { Hasse diagram } \\
\text { infinite coupling }\end{array}$ \\
\hline$E_{8}$ & $\begin{array}{c}2 k^{2}+9 k \\
\mathfrak{s o}(4 k+10)\end{array}$ & $\begin{array}{c}2 k^{2}+11 k+16 \\
\mathfrak{s o}(4 k+12)\end{array}$ & 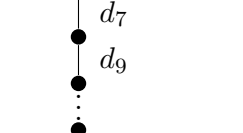 & \\
\hline$E_{7}$ & $\begin{array}{c}2 k^{2}+7 k \\
\mathfrak{s o}(4 k+8)\end{array}$ & $\begin{array}{c}2 k^{2}+7 k+8 \\
\mathfrak{s o}(4 k+8) \oplus \mathfrak{s u}(2)\end{array}$ & $d_{8}$ & $d_{80}$ \\
\hline$E_{6}$ & $\begin{array}{c}2 k^{2}+5 k \\
\mathfrak{s o}(4 k+6)\end{array}$ & $\begin{array}{c}2 k^{2}+5 k+4 \\
\mathfrak{s o}(4 k+6) \oplus \mathfrak{u}(1)\end{array}$ & $\stackrel{0}{\bullet}$ & \\
\hline$E_{5}$ & $\begin{array}{c}2 k^{2}+3 k \\
\mathfrak{s o}(4 k+4)\end{array}$ & $\begin{array}{c}2 k^{2}+3 k+2 \\
\mathfrak{s o}(4 k+4) \oplus \mathfrak{u}(1)\end{array}$ & $?$ & \\
\hline
\end{tabular}

Table 2. Coulomb branch quaternionic dimension, global symmetry and Hasse diagram for the orthogonal exceptional families, both at finite and infinite gauge coupling. The Hasse diagrams are further detailed in section 5 . Note the following changes between finite and infinite coupling. The constant terms in the dimension formulae change in powers of two, as indicated by the spinor representations for the free hypermultiplets in the $k=0$ case. The global symmetry at finite coupling does not include the $\mathrm{U}(1)_{I}$ factor since it is realized by the gaugino bilinear which lives in a nilpotent supermultiplet. At infinite coupling, it ceases to be nilpotent and becomes part of the geometry. As a consequence the rank of the global symmetry of the Higgs branch variety increases by one. The Hasse diagram is modified only in its top dimensional symplectic leaf for the $E_{6}$ and $E_{5}$ families, while the change is deeper for the $E_{7}$ and $E_{8}$ families. A recurring pattern for the $E_{n}$ families is the transformation of a $d_{n-1}$ transition into an $e_{n}$ transition.

for $\operatorname{Sp}(k)$ theories are studied from an alternative $\mathrm{O}^{-}$construction in section 4 , and contrasted to the results of section 3. Having derived the magnetic quivers for finite and infinite coupling Higgs branch in a variety of cases, section 5 details the derivation of the associated Hasse diagrams. In section 6, a family of linear orthosymplectic quiver gauge theories is studied and the multitude of different infinite coupling phases is discussed. Lastly, section 7 provides a conclusion and outlook. Appendix A provides background material on general Type II brane configurations with 8 supercharges, 5-brane webs, and $3 \mathrm{~d} \mathcal{N}=4$ Coulomb branches, as well as a brief summary of [80]. 


\begin{tabular}{|c|c|c|c|c|}
\hline Family & $\begin{array}{c}\text { Dimension and } \\
\text { symmetry for } k>1 \\
\text { at finite coupling }\end{array}$ & $\begin{array}{c}\text { Dimension and } \\
\text { symmetry for } k>1 \\
\text { at infinite coupling }\end{array}$ & $\begin{array}{l}\text { Hasse diagram } \\
\text { finite coupling }\end{array}$ & $\begin{array}{l}\text { Hasse diagram } \\
\text { infinite coupling }\end{array}$ \\
\hline$E_{4}$ & $\begin{array}{c}2 k^{2}+k \\
\mathfrak{s o}(4 k+2)\end{array}$ & $\begin{array}{c}2 k^{2}+k+1 \\
\mathfrak{s o}(4 k+2) \oplus \mathfrak{u}(1)\end{array}$ & & \\
\hline$E_{3}$ & $\begin{array}{c}2 k^{2}-k \\
\mathfrak{s o}(4 k)\end{array}$ & $\begin{array}{c}2 k^{2}-k+1 \\
\mathfrak{s o}(4 k) \oplus \mathfrak{u}(1)\end{array}$ & $d_{4}$ & \\
\hline $\begin{array}{l}E_{4-2 l} \\
k \geq l>0\end{array}$ & $\begin{array}{l}(k-l)(2 k-2 l+1) \\
\mathfrak{s o}(4 k-4 l+2)\end{array}$ & $\begin{array}{l}(k-l)(2 k-2 l+1)+1 \\
\mathfrak{s o}(4 k-4 l+2) \oplus \mathfrak{u}(1)\end{array}$ & $d_{3}$ & \\
\hline $\begin{array}{l}E_{3-2 l} \\
k \geq l>0\end{array}$ & $\begin{array}{c}(k-l)(2 k-2 l-1) \\
\mathfrak{s o}(4 k-4 l)\end{array}$ & $\begin{array}{l}(k-l)(2 k-2 l-1)+1 \\
\quad \mathfrak{s o}(4 k-4 l) \oplus \mathfrak{u}(1)\end{array}$ & $d_{4}$ & i \\
\hline
\end{tabular}

Table 3. Coulomb branch quaternionic dimension, global symmetry and Hasse diagram for the orthogonal exceptional families, both at finite and infinite gauge coupling. The Hasse diagrams are further detailed in section 5 . Note the following changes between finite and infinite coupling. The quaternionic dimension changes by one. The global symmetry at finite coupling does not include the $\mathrm{U}(1)_{I}$ factor since it is realized by the gaugino bilinear which lives in a nilpotent supermultiplet. At infinite coupling, it ceases to be nilpotent and becomes part of the geometry. As a consequence the rank of the global symmetry of the Higgs branch variety increases by one. The Hasse diagrams show, in some cases, a bifurcation; as detailed in section 5, the $\mathrm{O} 5$ brane construction studied in sections 2 and 3 only renders the part of the Hasse diagram depicted using vertical lines. The O7 brane construction studied in section 4 produces the entire Hasse diagrams. In the $E_{3}$ case, the bifurcation at the top of the finite coupling diagram could equivalently be denoted as $d_{2}$, and the bifurcation at the top of the infinite coupling diagram could equivalently be denoted as $e_{3}$, so that the modification at infinite coupling can be seen as $d_{2} \rightarrow e_{3}$. 


\begin{tabular}{|c|c|c|}
\hline Family & HWG at finite coupling & HWG at infinite coupling \\
\hline$E_{8}$ & $\sum_{i=1}^{k} \mu_{2 i} t^{2 i}$ & $\left(\sum_{i=1}^{k+2} \mu_{2 i} t^{2 i}\right)+t^{4}+\mu_{2 k+6}\left(t^{k+1}+t^{k+3}\right)$ \\
\hline$E_{7}$ & $\sum_{i=1}^{k} \mu_{2 i} t^{2 i}$ & $\begin{array}{c}\left(\sum_{i=1}^{k+1} \mu_{2 i} t^{2 i}\right)+\nu^{2} t^{2}+t^{4} \\
+\nu \mu_{2 k+4}\left(t^{k+1}+t^{k+3}\right)+\mu_{2 k+4}^{2} t^{2 k+4}-\nu^{2} \mu_{2 k+4}^{2} t^{2 k+6}\end{array}$ \\
\hline$E_{6}$ & $\sum_{i=1}^{k} \mu_{2 i} t^{2 i}$ & $\left(\sum_{i=1}^{k} \mu_{2 i} t^{2 i}\right)+t^{2}+\left(\mu_{2 k+2} q+\mu_{2 k+3} q^{-1}\right) t^{k+1}$ \\
\hline$E_{5}$ & $\sum_{i=1}^{k} \mu_{2 i} t^{2 i}$ & $\left(\sum_{i=1}^{k} \mu_{2 i} t^{2 i}\right)+t^{2}+\left(q+q^{-1}\right) \mu_{2 k+2} t^{k+1}$ \\
\hline$E_{4}$ & $\left(\sum_{i=1}^{k-1} \mu_{2 i} t^{2 i}\right)+\mu_{2 k} \mu_{2 k+1} t^{2 k}$ & $\begin{array}{c}\left(\sum_{i=1}^{k-1} \mu_{2 i} t^{2 i}\right)+t^{2}+\left(q \mu_{2 k}+q^{-1} \mu_{2 k+1}\right) t^{k+1} \\
+\mu_{2 k} \mu_{2 k+1}\left(t^{2 k}-t^{2 k+2}\right)\end{array}$ \\
\hline$E_{3}$ & $\begin{array}{c}\left(\sum_{i=1}^{k-1} \mu_{2 i} t^{2 i}\right)+\left(\mu_{2 k-1}^{2}+\mu_{2 k}^{2}\right) t^{2 k} \\
-\mu_{2 k-1}^{2} \mu_{2 k}^{2} t^{4 k}\end{array}$ & $\begin{array}{c}\left(\sum_{i=1}^{k-1} \mu_{2 i} t^{2 i}\right)+t^{2}+\left(q+\frac{1}{q}\right) \mu_{2 k} t^{k+1}+\mu_{2 k}^{2} t^{2 k} \\
-\mu_{2 k}^{2} t^{2 k+2}\end{array}$ \\
\hline$E_{4-2 l}$ & $\left(\sum_{i=1}^{k-l-1} \mu_{2 i} t^{2 i}\right)+\mu_{2 k-2 l} \mu_{2 k-2 l+1} t^{2 k-2 l}$ & 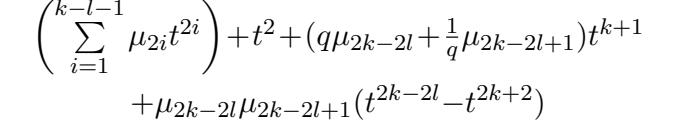 \\
\hline $\begin{array}{l}E_{3-2 l} \\
k \geq l \geq 0\end{array}$ & $\begin{array}{c}\left(\sum_{i=1}^{k-l-1} \mu_{2 i} t^{2 i}\right)+\left(\mu_{2 k-2 l-1}^{2}+\mu_{2 k-2 l}^{2}\right) t^{2 k-2 l} \\
-\mu_{2 k-2 l-1}^{2} \mu_{2 k-2 l}^{2} t^{4 k-2 l}\end{array}$ & $\begin{array}{c}\left(\sum_{i=1}^{k-l-1} \mu_{2 i} t^{2 i}\right)+t^{2}+\left(q+\frac{1}{q}\right) \mu_{2 k-2 l} t^{k+1} \\
+\mu_{2 k-2 l}^{2}\left(t^{2 k-2 l}-t^{2 k+2}\right)\end{array}$ \\
\hline
\end{tabular}

Table 4. Highest weight generating function (HWG) $[82,83]$ for the orthogonal exceptional families. The highest weight fugacities are assigned as follows: $\mu_{i}$ for $\mathfrak{s o}, \nu$ for $\mathfrak{s u}(2)$, and $q$ for $\mathfrak{u}(1)$. The global symmetry in each case can be read in tables 2 and 3 . Moreover, $t$ denotes the $\operatorname{SU}(2)_{R}$ fugacity along the $3 \mathrm{~d} \mathcal{N}=4$ Coulomb branch.

\section{Magnetic quivers and known examples}

\section{$2.1 \quad$ Set-up}

5-dimensional $\mathcal{N}=1$ gauge theories can be constructed as low-energy effective theories for 5-brane webs in Type IIB superstring theory [4-6]. The different branes occupy the space-time dimensions as summarised in table 5. Aiming for symplectic and orthogonal gauge symmetries, one may utilise orientifold planes [84, 85], in particular O5 planes [8-11] for the purposes of this work. As displayed in table 6, the effect is twofold: the gauge symmetry along 5-brane parallel to the O5 is projected to an orthogonal or symplectic algebra; while the flavour symmetry originating from transversal 7-branes is also projected to ortho-symplectic algebras, but in the opposite way. Further details on brane webs are summarised in appendix A. 


\begin{tabular}{|c|cccccccccc|}
\hline Type IIB & $x^{0}$ & $x^{1}$ & $x^{2}$ & $x^{3}$ & $x^{4}$ & $x^{5}$ & $x^{6}$ & $x^{7}$ & $x^{8}$ & $x^{9}$ \\
\hline NS5 & $\times$ & $\times$ & $\times$ & $\times$ & $\times$ & $\times$ & & & & \\
D5 & $\times$ & $\times$ & $\times$ & $\times$ & $\times$ & & $\times$ & & & \\
O5 & $\times$ & $\times$ & $\times$ & $\times$ & $\times$ & & $\times$ & & & \\
$(p, q)$ 5-brane & $\times$ & $\times$ & $\times$ & $\times$ & $\times$ & angle $\alpha$ & & & \\
{$[p, q]$ 7-brane } & $\times$ & $\times$ & $\times$ & $\times$ & $\times$ & & & $\times$ & $\times$ & $\times$ \\
\hline
\end{tabular}

Table 5. Type IIB 5-brane web set-up: $\times$ indicates the space-time directions spanned by the various branes and the orientifold plane. A $(p, q) 5$-brane is a line of slope $\tan (\alpha)=q \tau_{2} /\left(p+q \tau_{1}\right)$ in the $x^{5,6}$ plane where the axiodilaton is $\tau=\tau_{1}+i \tau_{2}$. In this paper all the brane webs are drawn for the value $\tau=i$, so that $\tan (\alpha)=q / p$.

\begin{tabular}{|c|c|c|c|c|}
\hline Orientifold & Drawing & Gauge algebra & Flavour algebra & Charge \\
\hline$\widetilde{\mathrm{O} p}$ & - & $B_{k}$ & $C_{n}$ & $\frac{1}{2}-2^{p-5}$ \\
$\mathrm{O} p^{+}$ & $\cdots \cdots \cdots$ & $C_{k}$ & $D_{n}$ & $2^{p-5}$ \\
$\widetilde{\mathrm{O} p}^{+}$ & $-\cdots$ & $C_{k}$ & $D_{n}$ & $2^{p-5}$ \\
$\mathrm{O} p^{-}$ & & $D_{k}$ & $C_{n}$ & $-2^{p-5}$ \\
\hline
\end{tabular}

Table 6. Summary of orientifolds. The gauge algebra is indicated for a stack of $k$ half $\mathrm{D} p$ branes on a $\mathrm{O} p$ orientifold plane. Whereas the flavour algebra is provided for a stack of $n$ half $\mathrm{D}(p+2)$ branes perpendicular to orientifold.

\subsection{Magnetic quivers}

A 5-brane web, where every external $(p, q) 5$-brane ends on a $[p, q] 7$-brane, in the presence of an O5 plane has various phases: for instance, the pure Coulomb branch phase, the pure Higgs branch phase, as well as mixed phases. As customary, the brane web in the massive Coulomb branch phase is reached by placing gauge and flavour D5 branes in various positions along the $x^{5}$ direction, i.e. assigning VEVs to scalars in the gauge multiplets as well as assigning masses to the fundamental flavours. This phase is convenient for reading off the electric gauge theory description via the suspension pattern of fundamental strings.

The pure Higgs branch phase at finite gauge coupling can be entered in two steps: firstly, the flavour D5 branes are moved to coincide on the orientifold as well, i.e. all mass parameters are set to zero. Secondly, all gauge D5 branes are aligned on the O5 plane, i.e. the VEVs of the $5 \mathrm{~d}$ gauge multiplet are tuned to zero. This results in a brane system which represents the origin of the Coulomb branch where Higgs branch directions can open up. For this, it is important to recall that the half $[1,0] 7$-branes, on which the flavour D5s end, merge with their mirrors on the orientifold plane, such that the resulting physical 7-brane can be split along the O5 plane. The S-rule then determines how many branes are connected between each half 7-brane. The Higgs branch degrees of freedom are realised by independent 5-brane subwebs that are free to move along the 7-branes in directions $x^{7}, x^{8}$, $x^{9}$. Each such subweb contributes one quaternionic dimension to the total Higgs branch. 
Besides the classical Higgs branch, meaning the Higgs branch of the low-energy effective gauge theory at finite gauge coupling, there exists also a Higgs branch at the RG-fixed point of the theory. For the purposes of this paper, the fixed point is always described by a $5 \mathrm{~d} \mathcal{N}=1 \mathrm{SCFT}$ and is further referred to as infinite (gauge) coupling phase. In terms of the 5-brane web, this point is reached when all external 5-branes meet at single point. The changes between the Higgs branch at finite and infinite coupling have been first emphasised in [1] and further elaborated in [10, 57, 58]. While [10] computed the change in Higgs branch dimension via 5-brane webs, it has been demonstrated in $[57,58]$ that infinite coupling Higgs branches can be described by Coulomb branches of $3 \mathrm{~d} \mathcal{N}=4$ gauge theories. Building on that, a systematic procedure on deriving the magnetic quiver for any phase of a given 5-brane web has been developed in [24]. The magnetic quiver proposal [24, conjecture 1] can be summarised as follows:

(i) Quiver: Find all inequivalent maximal subdivisions $\left\{\mathcal{S}_{i}\right\}$ of a given 5-brane web which is suspended between 7-branes. Whether a decomposition is compatible with supersymmetry can be determined by the S-rule [62], see also [86]. Each subdivision $\mathcal{S}_{i}$ gives rise to one magnetic quiver with the following data:

(ii) Gauge groups: The different subwebs in a given subdivision $\mathcal{S}_{i}$ are associated to magnetic gauge groups, wherein the number of identical subwebs determines the rank.

(iii) Matter: The magnetic hypermultiplets between two magnetic gauge groups are derived from the generalised intersection number of two different subwebs. This number is composed of intersection number of lines in the $x^{5,6}$ plane plus a modification if the subwebs end on common 7-branes.

Consequently, the Higgs branch associated to given phase of a 5-brane web is described via

$$
\mathcal{H}=\bigcup_{\left\{\mathcal{S}_{i}\right\}} \mathcal{C}\left(\mathrm{Q}\left(\mathcal{S}_{i}\right)\right)
$$

such that the moduli space is generically a union of symplectic singularities, one for each subdivison.

In order to derive magnetic quivers for 5-brane webs with 05 planes, the major conceptual challenge lies in the treatment of the orientifolds. In more detail, the T-dual set-up of [62], involving D3-D5-NS5 brane configurations, can accommodate for O3 planes [87] rather easily, because S-duality maps one O3 into another O3 plane. However, the same is not true for O5 planes, as S-duality, for instance, relates an ON plane with a O5 plane [88], see also [89]. Therefore, as emphasised in all magnetic quiver constructions [24, 60-62], S-duality is neither correct nor necessary to change between electric and magnetic theory. Consequently, the proposal of [61] is inspired from the O3 planes and involves magnetic orientifolds as summarise in table 7 . In other words, besides moving the 5-brane web into the Higgs branch phase, one also needs to convert the electric orientifolds into their magnetic counterparts in order to derive the magnetic quivers.

Before exploring potential predictions from magnetic quivers for 5-branes with orientifolds, one needs to derive a proposal that reproduces known cases. In section 2.3, the case of $5 \mathrm{~d} \mathcal{N}=1 \mathrm{SU}(2) \cong \mathrm{Sp}(1)$ gauge theory with $0 \leq N_{f}<8$ fundamental flavours is tested against the known symmetry enhancement at infinite coupling [1,2] and against 


\begin{tabular}{|c|c|c|c|}
\hline Orientifold & $\begin{array}{c}\text { Electric } \\
\text { algebra }\end{array}$ & $\begin{array}{c}\text { Magnetic } \\
\text { orientifold }\end{array}$ & $\begin{array}{c}\text { Magnetic } \\
\text { algebra }\end{array}$ \\
\hline $\mathrm{O} p^{+}$ & $C_{k}$ & $\widetilde{\mathrm{O} p}$ & $B_{k}$ \\
$\mathrm{O} p^{-}$ & $D_{k}$ & $\mathrm{O} p^{-}$ & $D_{k}$ \\
$\widetilde{\mathrm{O} p}+$ & $C_{k}$ & $\widetilde{\mathrm{O} p}^{+}$ & $C_{k}$ \\
$\widetilde{\mathrm{O} p}$ & $B_{k}$ & $\mathrm{O} p^{+}$ & $C_{k}$ \\
\hline
\end{tabular}

Table 7. Proposal for magnetic orientifolds [61]. A stack of $k$ physical D5 branes on top of a O5 plane, while being suspended between NS5 branes, has a 5-dimensional low-energy description given by a gauge field for the electric algebra. In contrast, $k$ identical subwebs suspended between 7-branes on top of a magnetic orientifold O5 contribute to the magnetic quiver as gauge multiplet for the magnetic algebra.

the magnetic quiver construction for 5-brane webs without orientifolds [24]. Thereafter, a precise proposal is formulated in section 2.4. One is then in the position to analyse various $5 \mathrm{~d}$ brane web constructions with this technique. In particular, section 3 is focused on the general case of $5 \mathrm{~d} \mathcal{N}=1 \mathrm{Sp}(k)$ gauge theory with $N_{f}$ fundamental flavours. As a proof of concept, a class of $5 \mathrm{~d} \mathcal{N}=1$ linear quiver gauge theories is analysed in section 6 .

\section{$2.3 \quad$ Single $\operatorname{Sp}(1)$ gauge group}

In this section, a $5 \mathrm{~d} \mathcal{N}=1 \mathrm{Sp}(1)$ gauge theory with $0 \leq N_{f}<8$ fundamental flavours is considered. The 5-brane web is presented, from which the magnetic quiver is derived at finite and infinite gauge coupling. A crucial consistency check is given by reproducing the enlargement of the Higgs branch from the minimal nilpotent orbit closure of $\mathfrak{s o}\left(2 N_{f}\right)$ at finite coupling to the minimal nilpotent orbit closure of $E_{N_{f}+1}$ at the UV fixed point. As detailed below, the finite coupling magnetic quivers agree with the results of [87].

\subsection{1 $\quad E_{8}: \operatorname{Sp}(1)$ with 7 flavours}

For the first case, consider $\mathrm{Sp}(1)$ with 7 flavours. The brane web in the Coulomb branch phase with massive flavours is given by

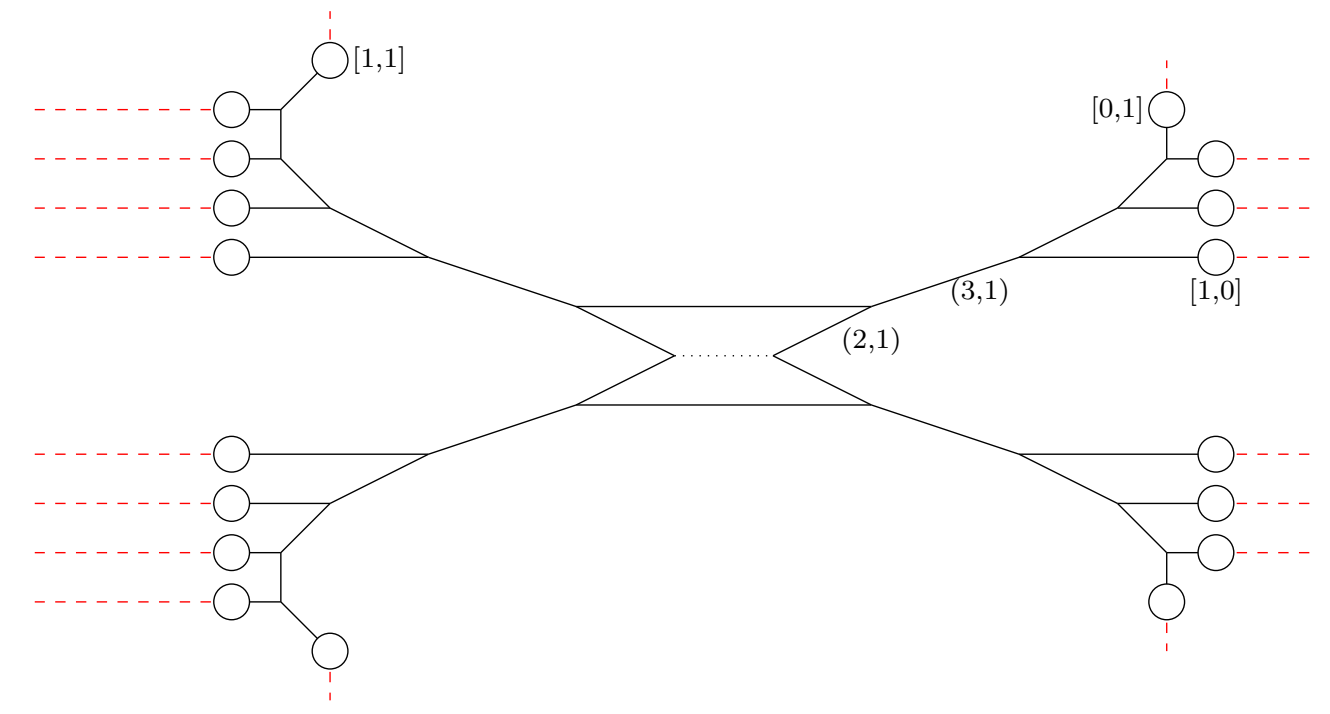


where the red dashed lines indicate the half monodromy cuts associated to each half 7brane. The orientifold notation is summarised in table 6. Moreover, the distance between the flavour 5-branes and the orientifold gives the bare mass. The transition into the Higgs branch, where all masses are set to zero, is achieved by two steps: firstly, aligning the flavour 7 -branes and the gauge $(1,0) 5$-brane on the orientifold. Secondly, the half 7 -branes merge with their mirror images such that the physical 7-branes can be split along the O5 plane.

The novelty, compared to the classical 5-brane web construction with $N_{f}<7$, is that one of the external non-flavour branes bends inside, i.e. the $(1,1) 5$-brane. Consequently, there are two intermediate representations of the Higgs branch phase. On the one hand, the non-flavour branes are kept separated such that the brane web (2.2) turns into

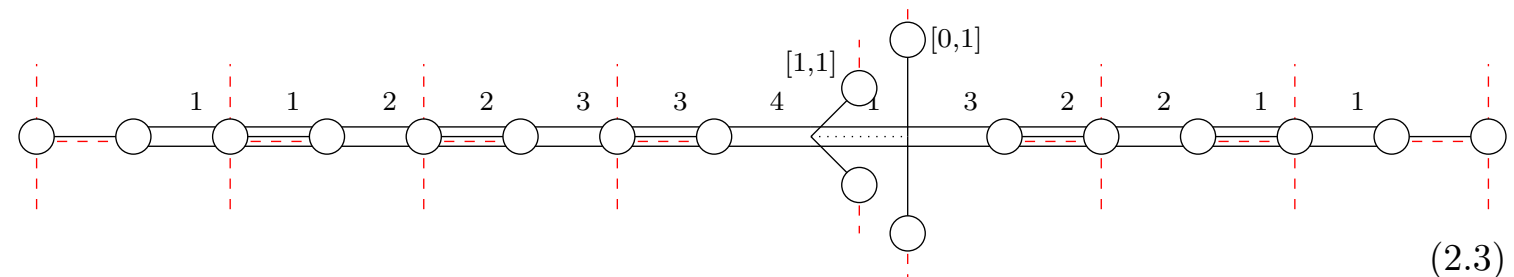

The numbers displayed above the branes count physical $(1,0) 5$-branes on top of O5 planes, see table 6 for conventions.

On the other hand, the $(1,0)$ and $(1,1)$ 5-branes can cross such that the brane configuration becomes

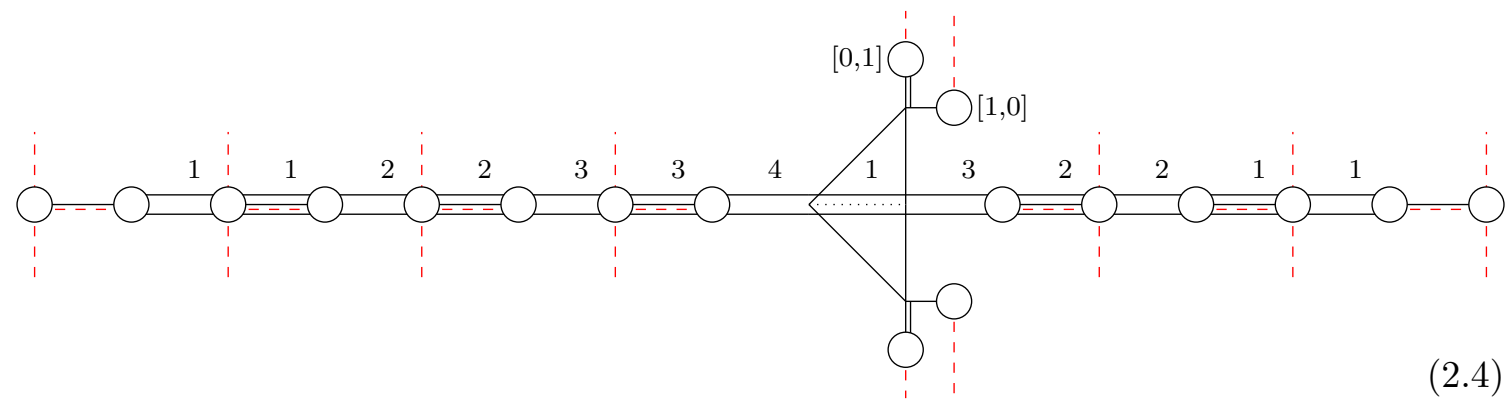

where one accounts for brane creation, see appendix A. Both, (2.3) and (2.4), are useful starting points as shown below.

Finite coupling. To analyse the finite coupling Higgs branch, one considers (2.3) and notices that several branes are non-dynamical as they serve to connect the $(0,1)$ and $(1,1)$ 5 -brane with several half 7 -branes in order to render the configuration supersymmetric. The number of these branes can be minimised by transitioning the $(0,1)$ and $(1,1)$ through several half 7-branes such that brane annihilation leads to the following configuration:

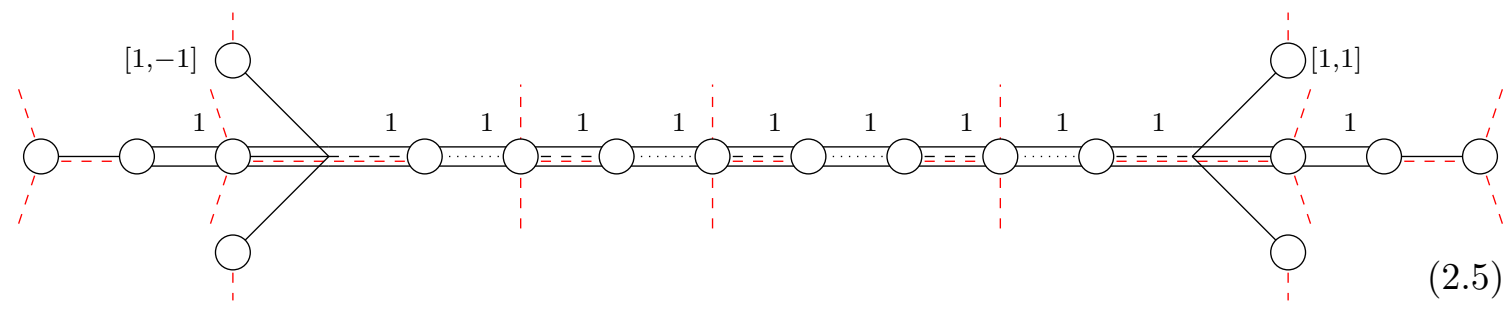

where the monodromy cuts have been chosen for unambiguous presentation. 
Starting from (2.5), the magnetic quiver is read off as follows: to begin with, the orientifolds are converted into magnetic orientifolds, see table 7 . Then one needs to find all maximal subdivisions: in this case, there is only one. Between the half 7-branes along the orientifold plane, all physical D5 branes constitute independent subwebs. Note that there are no two identical subwebs, because the D5s are suspended between different 7-branes. Consequently, all magnetic gauge groups associated to these branes have rank one because each subweb contributes one quaternionic dimension to the Higgs branch. The character of the magnetic gauge multiplet is deduced from the magnetic orientifold between two adjacent $[1,0]$-branes. The remaining parts that need to be understood are the $[1, \pm 1]$ 5 -branes on the left and right-hand-side. To be specific focus on the left-hand-side, one could transition the $(1,-1)$ 5-brane through one more half 7 -brane to the left without brane creation or annihilation. Due to the effects of the monodromy line, one would end up with a $(2,-1) 5$-brane, which is known to be a supersymmetric configuration by itself, see (A.2). Therefore, the original $(1,-1) 5$-brane is also supersymmetric, but, in contrast to all other 5 -branes, the $(1,-1) 5$-brane is not an independent subweb. In particular, this means that both the $(1, \pm 1)$ branes are non-dynamical as these cannot be moved along the $x^{7,8,9}$ directions. As such these pieces of the brane web contribute as magnetic $\mathrm{B}_{0}$ flavour nodes only. Analogous to [61, eq. (2.66)], the $\mathrm{B}_{0}$ denotes a single half-hypermultiplet for a $\mathrm{C}_{k}$ magnetic gauge node. As a remark, for reading off the magnetic gauge algebra, it is convenient to have the free consistent configuration on top of $\widetilde{\mathrm{O} 5}^{+}$and $\widetilde{\mathrm{O} 5}^{-}$planes, because the magnetic orientifolds of table 7 imply an unambiguous magnetic algebra in contrast to a configuration on top of $\mathrm{O}^{+}$and $\mathrm{O}^{-}$planes.

This discussion reveals the magnetic gauge multiplets and background gauge multiplets. In addition, there is matter derived from the generalised intersection number [24]. To be explicit, consider two adjacent D5 branes connecting a common half 7-brane from the left and right-hand-side, respectively. Then the intersection number between the two D5s is zero, but there is a positive contribution because they end on the common 7-brane from opposite sides. The generalised intersection number turns out to be one. Alternatively, one can simply consider D3 branes suspended between the two subwebs and recognise the bifundamental hypermultiplet between the gauge groups, see also [61]. Lastly, consider the $(1, \pm 1)$ branes together with the closest D5 brane. Here, one computes the intersection number between a $(1, \pm 1)$ and a $(1,0) 5$-brane, which is simply one. Since there are no common 7-branes, the generalised intersection number is one too, indicating a simple magnetic fundamental hypermultiplet between the flavour and gauge node.

Collecting all the pieces, the magnetic quiver becomes

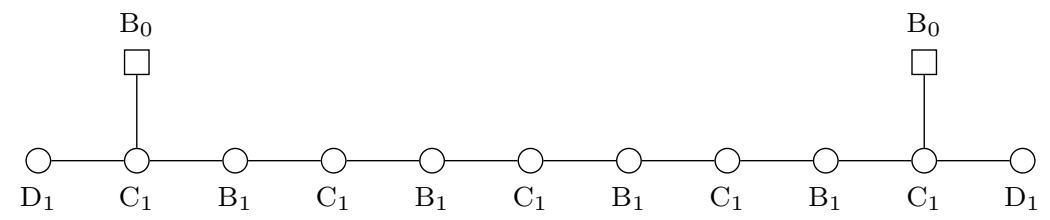

such that Coulomb branch dimension and global symmetry, see appendix A.3, are computed to be

$$
\operatorname{dim}_{\mathbb{H}} \mathcal{C}(2.6)=11, \quad G=\mathrm{SO}(14)
$$


Upon choosing the magnetic gauge groups suitably, the full Coulomb branch geometry is known [90-92]

$$
\mathcal{C}(2.6)=\overline{\mathcal{O}}_{\mathrm{D}_{7}}^{\min },
$$

i.e. the closure of the minimal nilpotent orbit of $\mathfrak{s o}(14)$. The details of the assignment of the magnetic gauge group are the subject of the companion paper [80], see also appendix A.4. The properties (2.7) match the classical Higgs branch as well as the non-abelian part of the global symmetry.

The alert reader will notice that the $\mathrm{U}(1)_{I}$ symmetry is missing from the classical global symmetry computed from the magnetic quiver. This is because this global symmetry is associated with the gaugino bilinear. Being a nilpotent operator in the chiral ring, the gaugino bilinear does not enter the geometric part of the classical moduli space, called Higgs variety in [59]. The current status of magnetic quivers is not sensitive to nilpotent elements, i.e. it is does not compute the whole Higgs Scheme. If nilpotent operators are present in the chiral ring, the Higgs branch is a non-reduced scheme, and the magnetic quiver computes its geometric reduction. See [57] for a discussion of the gaugino biliniear and [59] for a detailed analysis of the difference between the Higgs variety and the Higgs scheme.

Infinite coupling. For the brane web at infinite coupling, the configuration (2.4) is convenient. Here, moving the infinite coupling is realised by moving the half $[1,0] 7$-brane, which is away from the orientifold, onto the $\mathrm{O} 5$ plane. Then it merges with its mirror image and the resulting physical 7-brane can be split along the orientifold. As a consequence, the brane web becomes

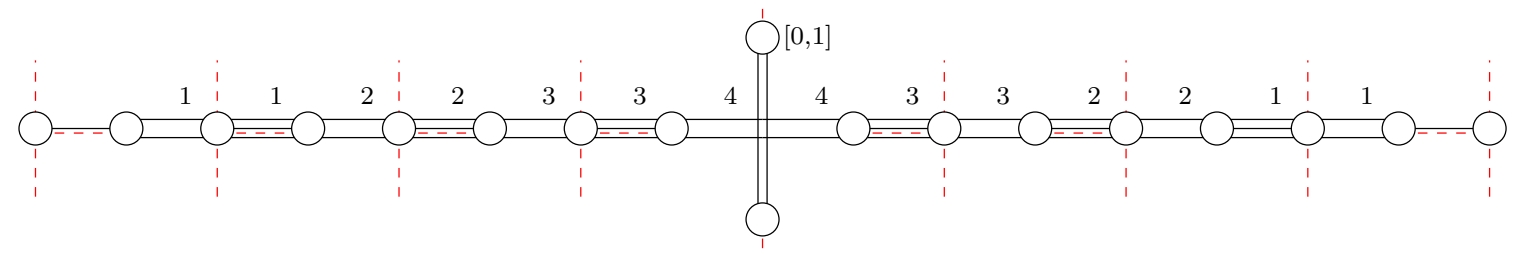

and, again, the numbers denote physical D5 branes in between half 7-branes. The magnetic quiver is derived as follows: inspecting (2.8) shows that there is only one maximal subdivision. Therein, all subwebs are given either by D5 branes suspended between adjacent 7-branes along the orientifold or by NS5 branes suspended between a half 7-brane and its mirror image away from the $\mathrm{O} 5$ plane. There are two new features compared to the finite coupling case: firstly, there are multiple copies of identical subwebs. The number of physical D5 branes between two adjacent half 7-branes coincides with the number of identical webs, and, therefore, yields the rank of the magnetic gauge group after transition to the magnetic orientifold, see table 7. Secondly, the NS5 branes also form one subweb, but the orientifold projections now acts differently. Since the magnetic orientifold for the NS5s is a $\mathrm{O}^{-}$plane, the NS5 branes contribute a $\mathrm{C}_{1}$ magnetic gauge group.

The determination of the matter content proceeds as above: the stacks of adjacent D5 brane subwebs have generalised intersection number of one; while the NS5 branes and 
the D5 branes in the central segment have intersection number one with no additional corrections from common 7-branes. Therefore, all magnetic gauge nodes are connected by bifundamental magnetic hypermultilets. Alternatively, the same conclusion is reached by inspecting the suspension pattern of D3 branes, analogous to the $6 \mathrm{~d}$ setting of [61].

Consequently, the magnetic quiver associated to (2.8) is given by

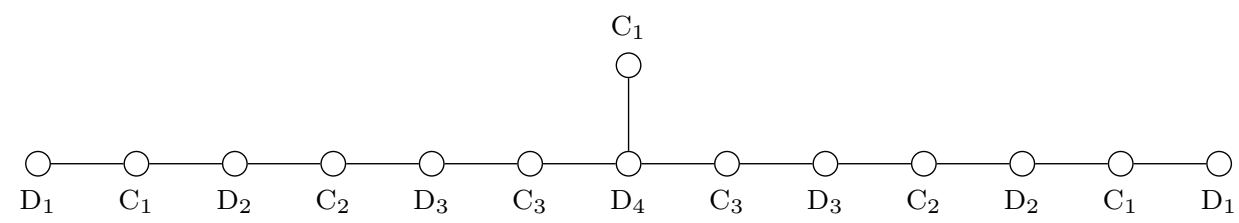

with a Coulomb branch dimension of

$$
\operatorname{dim}_{\mathbb{H}} \mathcal{C}(2.9)=29
$$

It has been concluded in [65, eq. (2.43)] and [93] that the Coulomb branch of (2.8) is the minimal nilpotent orbit of $E_{8}$. However, this result relies on clarifying what magnetic gauge group has to be assigned to (2.9), which is elaborated on in a companion paper [80] see appendix A.4 for a summary).

Comparing to class $\mathcal{S}$ technology, one can decompose the star-shaped quiver (2.9) into three $D_{4}$ punctures associated with the following linear quivers: $T_{\left(1^{8}\right)}[\mathrm{SO}(8)], T_{\left(1^{8}\right)}[\mathrm{SO}(8)]$, and $T_{(5,3)}[\mathrm{SO}(8)]$. As demonstrated in [78, section 3.2.2], the fixture obtained via gluing gives rise to the so-called $E_{8} \mathrm{SCFT}$, which yields further validation to the approach of this paper.

\subsection{2 $E_{7}: \operatorname{Sp}(1)$ with 6 flavours}

Considering $\operatorname{Sp}(1)$ with 6 fundamental flavours, the brane web in the Coulomb branch phase with massive flavours is given by

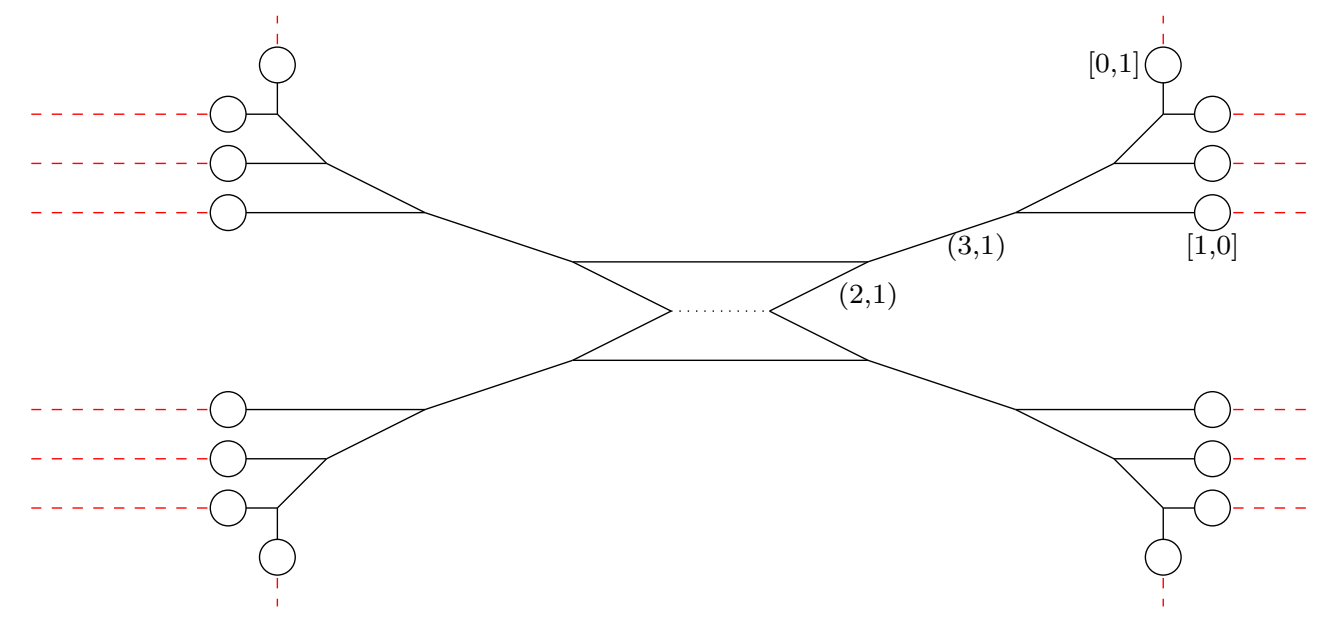

where the semi-infinite flavour 5-branes are set to end on half 7 -branes. Next, the Higgs branch phase is reached by moving the half $[1,0] 7$-branes and the $(1,0)$ gauge 5 -brane onto 
the O5 plane. The half 7-branes merge with their mirror images such that the resulting physical 7-brane can be split along the orientifold. The brane web becomes

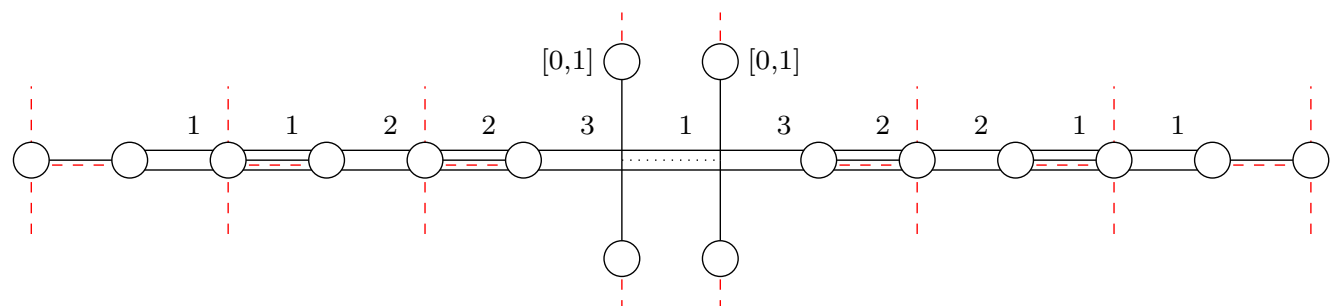

and the S-rule implies that the two $(0,1) 5$-branes need to be connected to more than one 7 -brane via $(1,0) 5$-branes.

Finite coupling. The non-dynamical parts of the brane web (2.12) can be minimised by transitioning the $(0,1) 5$-branes to the left or right, respectively. Accounting for brane annihilation leads to

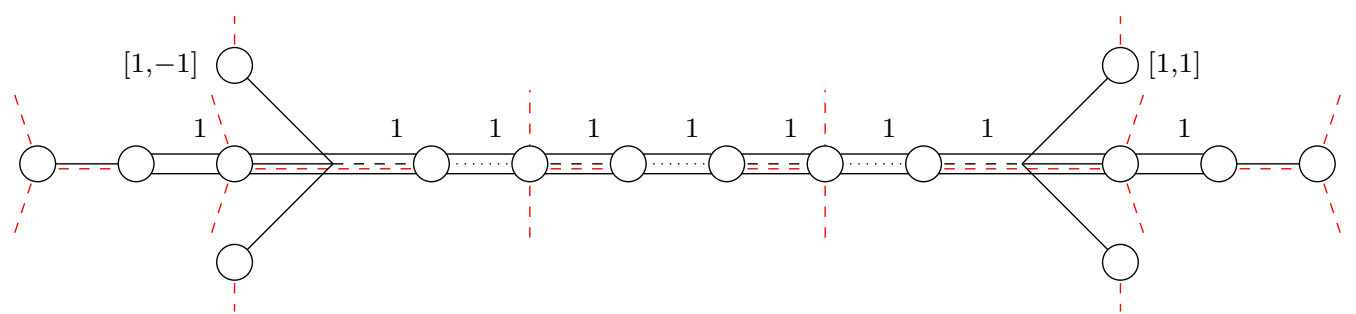

where the monodromy cuts have been chosen for the sake of a clear presentation. The only maximal subdivision is composed of subwebs that are D5 branes suspended between adjacent half 7 -branes. As above, the $(1, \pm 1)$ 5-branes between $\widetilde{\mathrm{O}}^{+}$and $\widetilde{\mathrm{O} 5}^{-}$are supersymmetric configurations by themselves. However, these branes are non-dynamical, as they are not part of any subweb in a maximal subdivision of (2.13). The magnetic quiver associated to (2.13) is read off by the same logic as above; hence, one finds

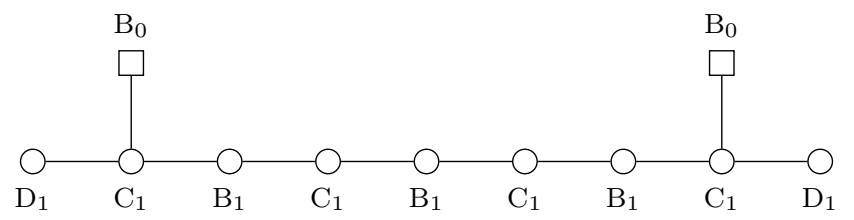

and Coulomb branch dimension and global symmetry, see appendix A.3, are given by

$$
\operatorname{dim}_{\mathbb{H}} \mathcal{C}(2.14)=9, \quad G=\mathrm{SO}(12) .
$$

Even more is true, because if the magnetic gauge groups for the quiver (2.14) are chosen appropriately then it is known [90-92] that

$$
\mathcal{C}(2.14)=\overline{\mathcal{O}}_{\mathrm{D}_{6}}^{\min },
$$

which denotes the closure of the minimal nilpotent orbit of $\mathfrak{s o}(12)$. Again, the correct choice of magnetic gauge group is subject of the companion paper [80]. Compared to the classical Higgs branch and the non-abelian part of the global symmetry, the magnetic quiver matches these properties (2.15) exactly. 
Infinite coupling. Proceeding to infinite coupling means that the two $(0,1)$ 5-branes in (2.12) coincide on the O5 plane. Since both branes end on half 7-branes, these can be vertically displaced slightly to a brane configuration of the form

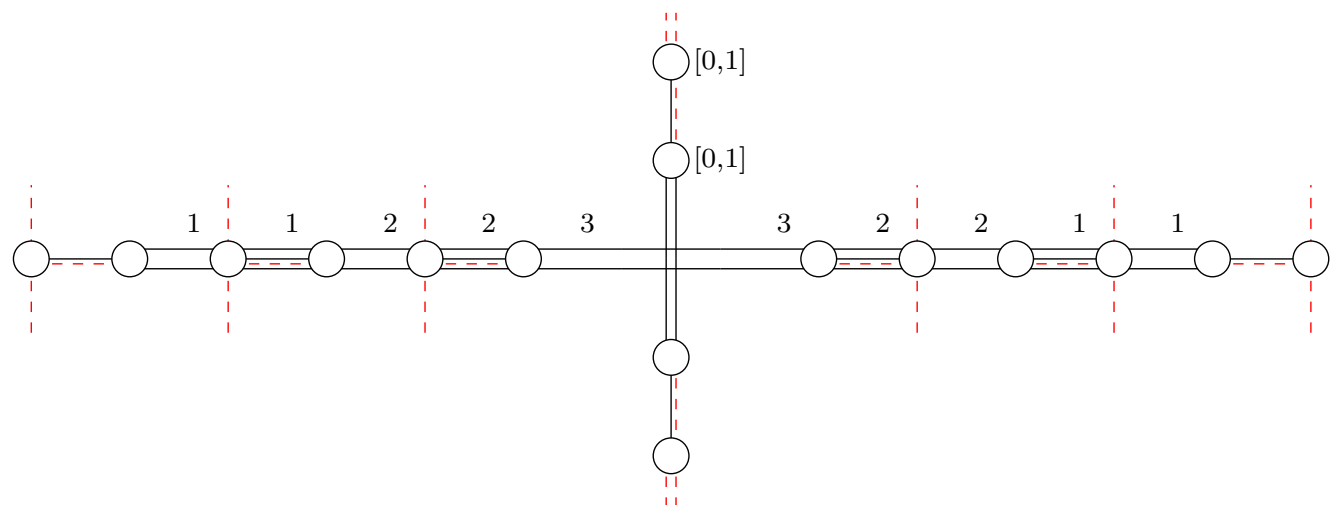

Finding a maximal subdivision is similar to the cases above, the only new feature is the set of vertically aligned half 7 -branes outside the O5 plane. The two (half) $(0,1) 5$-branes that pass through the orientifold form two identical subwebs that are subjected to the projections of the magnetic orientifolds as discussed above. The $(0,1) 5$-brane that is suspended between the two half $[0,1] 7$-branes outside the orientifold is, in contrast, not affected by the orientifold projection and, consequently, induces a unitary magnetic gauge multiplet of rank one. The matter content between the magnetic $\mathrm{U}(1)$ and $\mathrm{C}_{1}$ node is determined by the intersection property. The intersection number is zero, but the generalised intersection number receives a positive contribution because the corresponding subwebs end on opposite side on the common half 7-brane. Therefore, there exists a magnetic bifundamental hypermultiplet between these nodes.

Adding all the pieces, one then derives the following magnetic quiver:

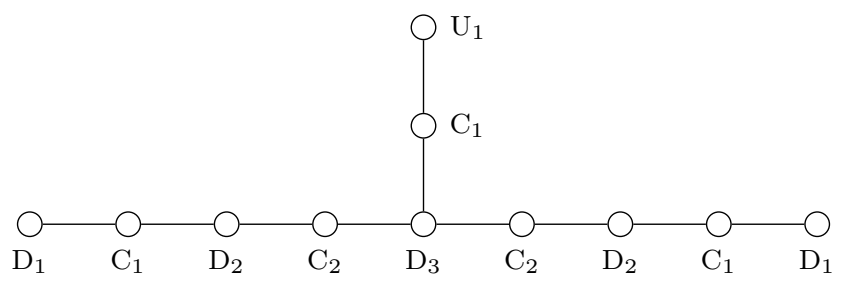

and the Coulomb branch dimension is computed to be

$$
\operatorname{dim}_{\mathbb{H}} \mathcal{C}(2.17)=17 \text {. }
$$

The physical choice of magnetic gauge groups for any orthosymplectic quiver is subject of the companion paper [80]; such that a Hilbert series analysis suggests that $\mathcal{C}(2.17)=\overline{\mathcal{O}}_{E_{7}}^{\text {min }}$ holds.

Borrowing from class $\mathcal{S}$ literature, the star-shaped quiver (2.17) can be decomposed into three $D_{3}$ punctures corresponding to the linear quivers $T_{\left(1^{6}\right)}[\mathrm{SO}(6)], T_{\left(1^{6}\right)}[\mathrm{SO}(6)]$, and $T_{\left(3,1^{3}\right)}[\mathrm{SO}(6)]$. As detailed in [78, section 3.2.2], the resulting fixture gives rise to the so-called $E_{7}$ SCFT. 


\subsection{3 $E_{6}: \operatorname{Sp}(1)$ with 5 flavours}

The 5-brane web for $\mathrm{Sp}(1)$ with 5 fundamental flavours in the Coulomb branch phase with masses for the flavours is given by

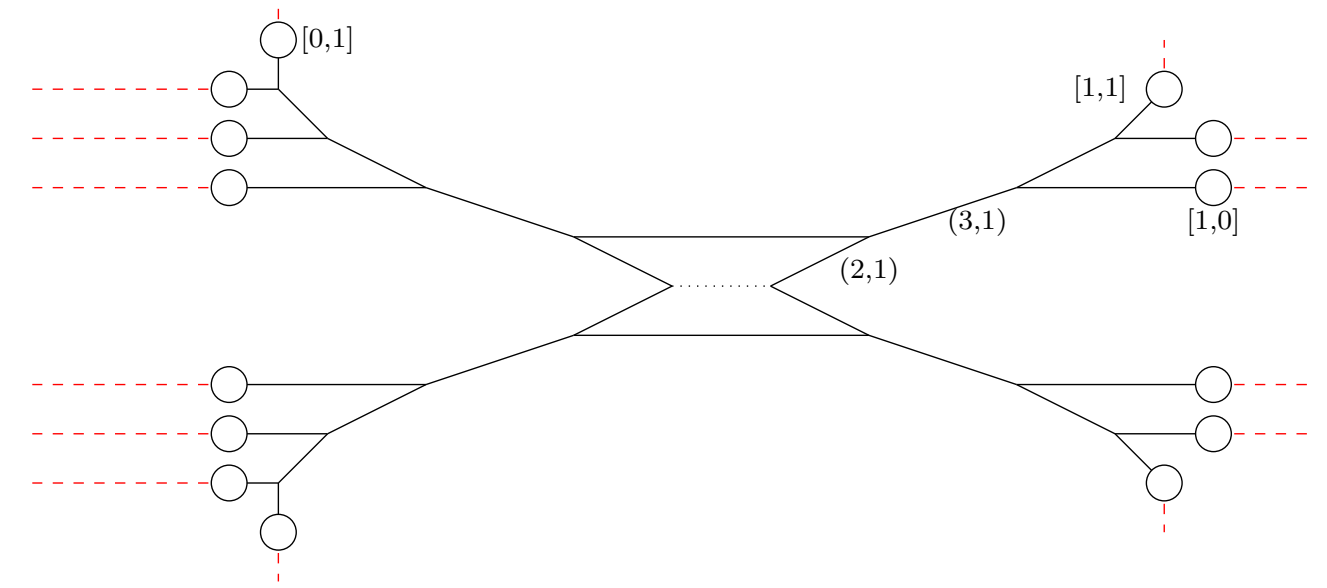

and the splitting into 3 flavours on the left and 2 flavours on the right-hand-side is a convenient choice. Subsequently moving to the origin of the $5 \mathrm{~d}$ Coulomb branch is realised by moving the flavour half $[1,0] 7$-branes and the gauge $(1,0) 5$-brane onto the $\mathrm{O} 5$ plane. Once the half 7-branes merge with their respective mirror branes, the physical 7-branes can split on the orientifold leading to the following brane web in the Higgs branch phase:

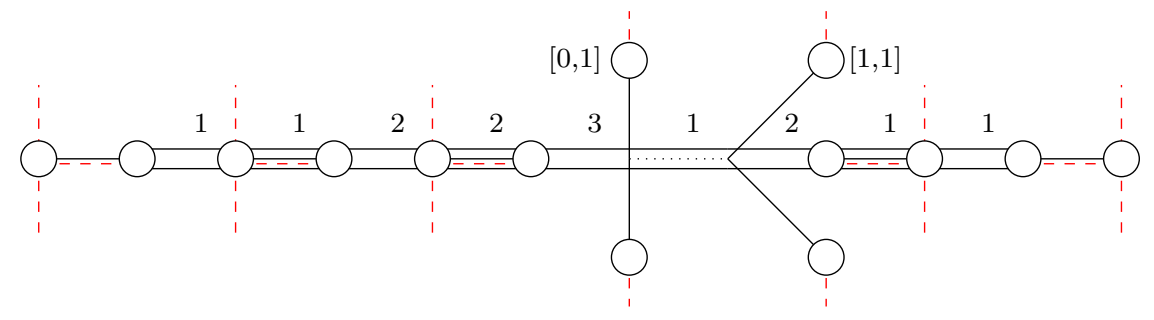

The $(1,1) 5$-brane is connected via another $(1,0) 5$-brane to a 7 -brane on the right-handside, due to the S-rule. Moreover, the $(0,1) 5$-brane has to be connected to several 7-branes on the left-hand-side in order to preserve supersymmetry.

Finite coupling. To analyse the finite coupling Higgs branch, one eliminates the nondynamical 5-branes as much as possible by transitioning the $(0,1)$ and $(1,1) 5$-brane through 7 -branes on the left or right, respectively. Carefully considering brane annihilation reveals that the configuration (2.20) becomes

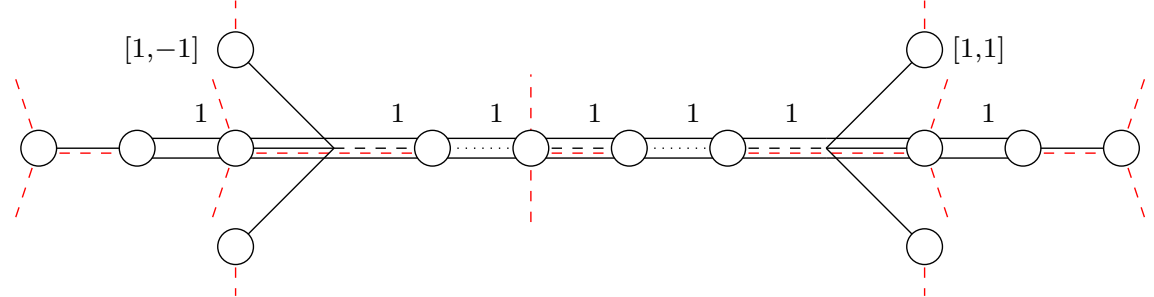


where the monodromy cuts have been chosen for an unambiguous presentation. As above the $(1, \pm 1)$ 5-branes between $\widetilde{\mathrm{O} 5}^{+}$and $\widetilde{\mathrm{O} 5}^{-}$are supersymmetric, but non-dynamical branes. Hence, these contribute as flavours to the magnetic quiver. The remaining brane web is subdivided into subwebs, where each $(1,0) 5$-brane ending on 7 -branes is such a consistent subweb. Then, following table 7 , the magnetic quiver associated to (2.21) is given by

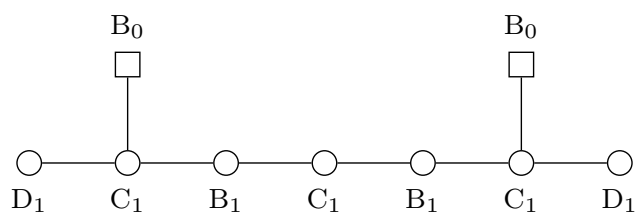

and the Coulomb branch dimension and global symmetry, see appendix A.3, can be computed to be

$$
\operatorname{dim}_{\mathbb{H}} \mathcal{C}(2.22)=7 ; \quad G=\mathrm{SO}(10)
$$

Upon choosing the magnetic gauge groups for (2.22) suitably, the entire moduli spaces is known [92, table 11], see also [90, 91],

$$
\mathcal{C}(2.22)=\overline{\mathcal{O}}_{\mathrm{D}_{5}}^{\min }
$$

i.e. the closure of the minimal nilpotent orbit of $\mathfrak{s o}(10)$. The rationale behind the choice of magnetic gauge group for orthosymplectic magnetic quivers is addressed in a companion paper [80]. Consequently, the properties (2.23) correctly reproduce the finite coupling Higgs branch as well as the non-abelian part of the global symmetry.

Infinite coupling. In order to transition to the Higgs branch phase at infinite coupling, the $(0,1)$ and $(1,1) 5$-brane in $(2.20)$ have to become coincident on the O5 plane. However, as remarked above, there is a non-dynamical brane in (2.20), which can be eliminated by passing the $(0,1)$ and $(1,1) 5$-brane through one half 7 -brane on the left-hand-side. Accounting for brane-annihilation leads to

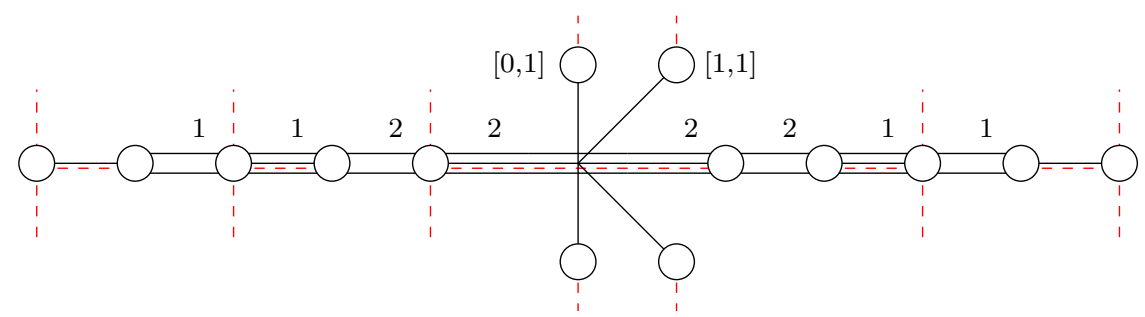

where the $(1,1)$ and $(0,1) 5$-branes constitute an independent subweb in the maximal subdivision of (2.24), see also appendix A. Again, this subweb passes through the O5 plane, but is mapped to itself, such that the magnetic degrees of freedom are not affected by the orientifold projection. The subweb contributes a unitary magnetic vector multiplet. The generalised intersection number between this subweb and the D5 branes in the central segment is computed to be one, simply from the intersection number without further corrections. Therefore, the $\mathrm{U}(1)$ node connects via a bifundamental magnetic hypermultiplet 
to another magnetic gauge node. In addition, since the $(0,1)$ and $(1,1) 5$-brane have a nontrivial intersection before orientifolding, one may wonder if this implies additional matter. However, due to the identification of the 7-branes which leads to common 7-branes, the generalised intersection evaluates to zero and no matter multiplet arises.

The remaining subwebs are, again, given by D5 branes between half 7-branes and represent no new challenge. Accounting for identical subwebs and transitioning to magnetic orientifolds, see table 7 , leads to the following magnetic quiver:

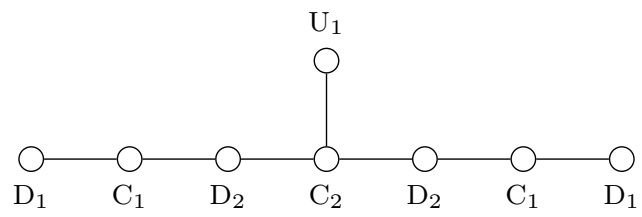

The Coulomb branch dimension is computed to be

$$
\operatorname{dim}_{\mathbb{H}} \mathcal{C}(2.25)=11 .
$$

The identification of the magnetic gauge groups for (2.25) is addressed in the companion paper [80], such that a Hilbert series computation indicates the stronger claim $\mathcal{C}(2.25)=\overline{\mathcal{O}}_{E_{6}}^{\text {min }}$.

In terms of the class $\mathcal{S}$ dictionary, the star-shaped quiver (2.25) can be decomposed into one untwisted $A_{3}$ puncture corresponding to the linear quiver $T_{(3,1)}[\mathrm{SU}(4)]$ and two twisted $A_{3}$ punctures associated with the linear quiver $T_{\left(1^{5}\right)}[\mathrm{USp}(4)]$. The resulting twisted $A_{3}$ fixture is referred to as $E_{6} \operatorname{SCFT}[79$, section A.1.5].

\subsection{4 $E_{5}: \operatorname{Sp}(1)$ with 4 flavours}

The 5-brane web for $\mathrm{Sp}(1)$ with 4 massive flavours in the Coulomb branch phase reads

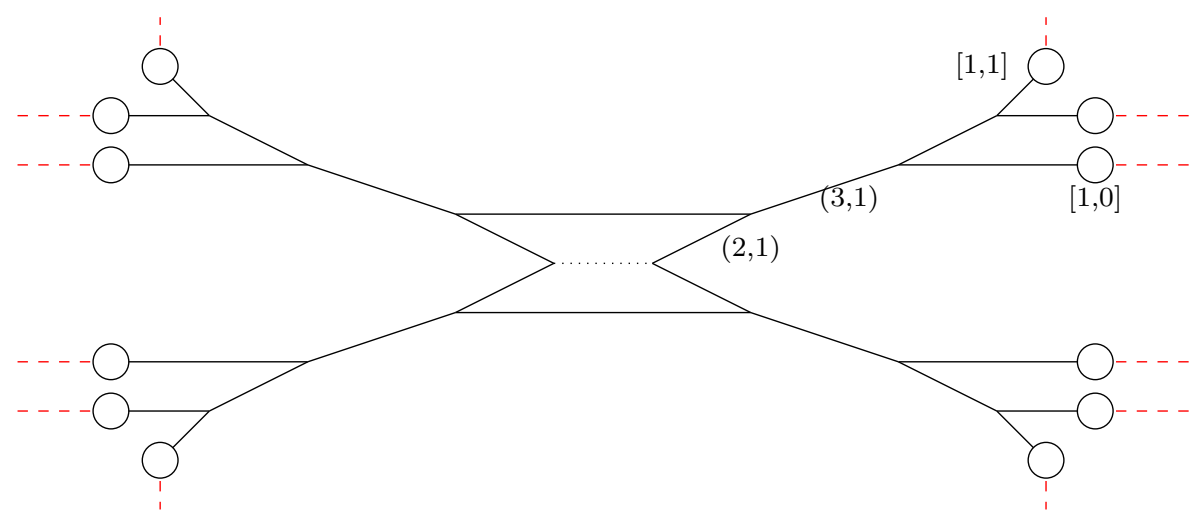

and the flavour branes have been chosen symmetrically for convenience. Transitioning to the Higgs branch phase, configuration (2.27) becomes

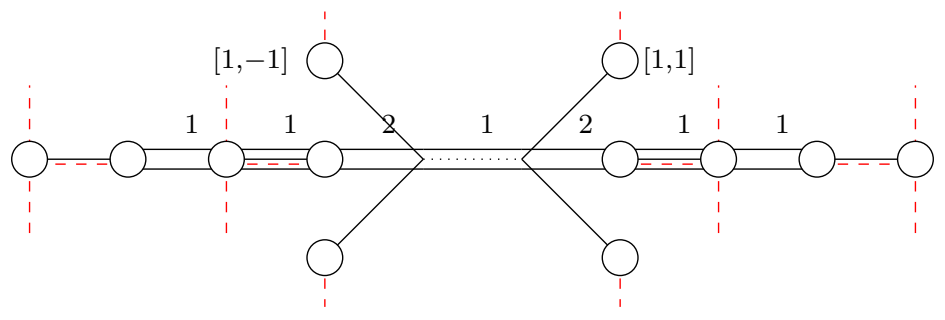


As above, the $(1, \pm 1) 5$-branes each need to be connected to a $[1,0] 7$-brane via a $(1,0)$ 5 -brane for consistency.

Finite coupling. The finite coupling Higgs branch phase is most conveniently analysed when the $(1, \pm 1)$ 5-branes in $(2.28)$ are passed through the 7-branes on the left or right-hand-side, respectively. Accounting for brane annihilation, the resulting 5-brane web becomes

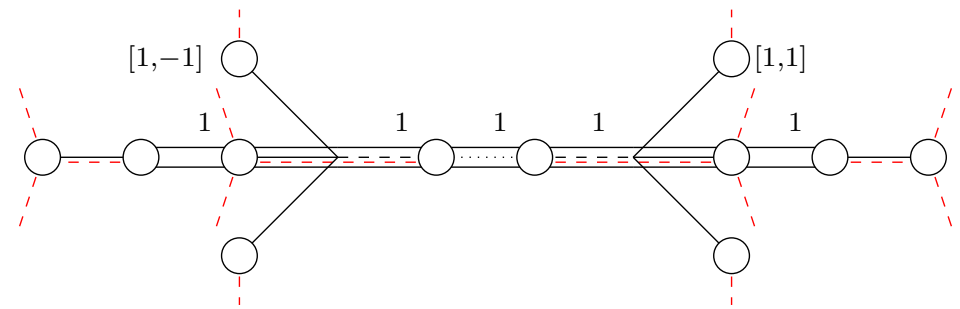

where the monodormy cuts have been slightly displaced for the sake of an unambiguous configuration. Now, one can analyse the Higgs branch degrees of freedom. The $(1, \pm 1)$ 5 -branes between $\widetilde{\mathrm{O} 5}^{+}$and $\widetilde{\mathrm{O}} 5^{-}$are not freely-moving subwebs, and contribute only as magnetic flavours. The $(1,0) 5$-branes between the different 7-branes are all subwebs and induce magnetic gauge multiplets according to which orientifold they coincide with, see table 7 . The associated magnetic quiver reads

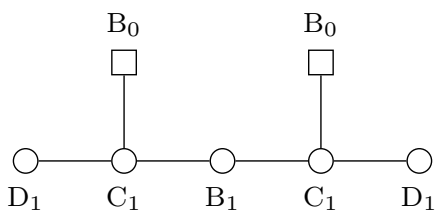

and Coulomb branch dimension and global symmetry, see appendix A.3, are computed to be

$$
\operatorname{dim}_{\mathbb{H}} \mathcal{C}(2.30)=5, \quad G=\mathrm{SO}(8) .
$$

In fact, it is known [92, table 7], see also [90, 91], that upon choosing the magnetic gauge groups corresponding to the algebras in (2.30) correctly,

$$
\mathcal{C}(2.30)=\overline{\mathcal{O}}_{\mathrm{D}_{4}}^{\min }
$$

meaning that (2.30) describes the closure of the minimal nilpotent orbit of $\mathfrak{s o}(8)$. The reasoning behind choosing the magnetic gauge group from an orthosynmplectic magnetic quiver is subject of a companion paper [80]. Consequently, the properties (2.31) correctly match the classical Higgs branch as well as the non-abelian part of the global symmetry.

Infinite coupling. The brane configuration for infinite gauge coupling is reached by starting from $(2.28)$ and making the $(1, \pm 1) 5$-branes become coincident on the $\mathrm{O} 5$ plane. 
In more detail

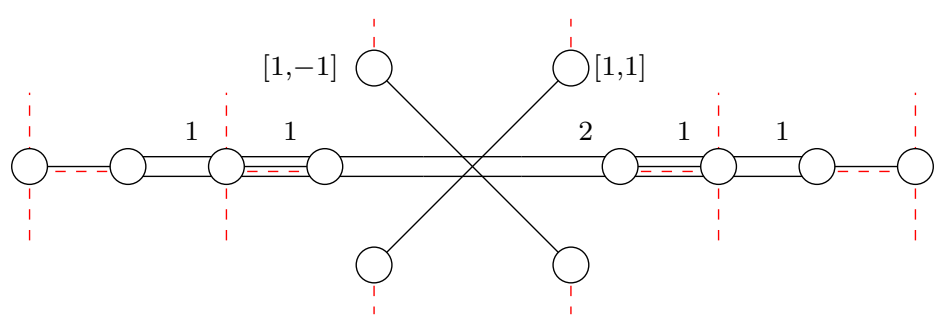

such that a new Higgs branch degree of freedom appears because the $(1, \pm 1)$ 5-branes form an independent subweb. In contrast to the subwebs formed by D5 branes suspended between $[1,0] 7$-branes, the subweb given by the $(1, \pm 1)$ branes passes through the $\mathrm{O} 5$ plane, but is mapped to itself under the orientfold action. Hence, the O5 plane does not affect the magnetic degrees of freedom of this subweb, such that the contribution is a unitary magnetic gauge node as in [24]. Without the O5 plane, the $(1, \pm 1) 5$-branes have a nontrivial intersection, but the orientifold identifies the 7-branes on which the 5-brane ends. Thus, the generalised intersection evaluates to zero because of the negative contributions from the common 7-brane, such that no new matter multiplet arises.

Using the prescription of the magnetic orientifolds of table 7 for the remaining subwebs of D5 branes and computing the intersection numbers, the magnetic quiver is read off to be

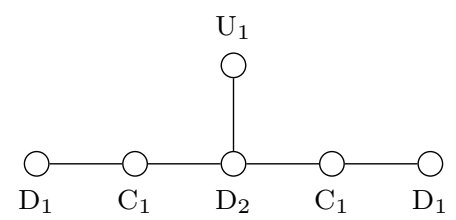

with a Coulomb branch of dimension

$$
\operatorname{dim}_{\mathbb{H}} \mathcal{C}(2.33)=7
$$

In a companion paper [80], the choice of magnetic gauge group for (2.33) will be clarified, which then indicates that $\mathcal{C}(2.33)=\overline{\mathcal{O}}_{E_{5}}^{\min }$ from a Hilbert series analysis.

\subsection{5 $E_{4}: \operatorname{Sp}(1)$ with 3 flavours}

The 5-brane web for $\operatorname{Sp}(1)$ with 3 fundamental flavours can be chosen to be

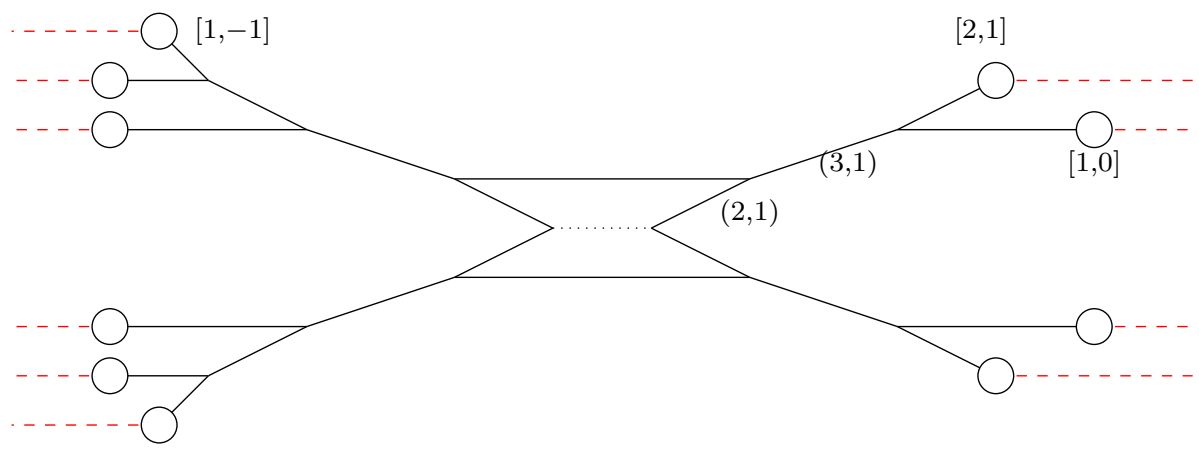


where the separation into 2 flavours on the left and 1 flavour on the right is simply a convenient choice. In order to transfer the 5-brane web (2.35) into the Higgs phase, one moves the system to the origin of the $5 \mathrm{~d}$ Coulomb branch which is reached when the flavour $[1,0] 7$-branes and the gauge $(1,0) 5$-brane are on the 05 plane. After merging the half $[1,0] 7$-branes with their mirrors on the orientifold and subsequent splitting along the O5 plane, the Higgs branch phase for (2.35) is given by

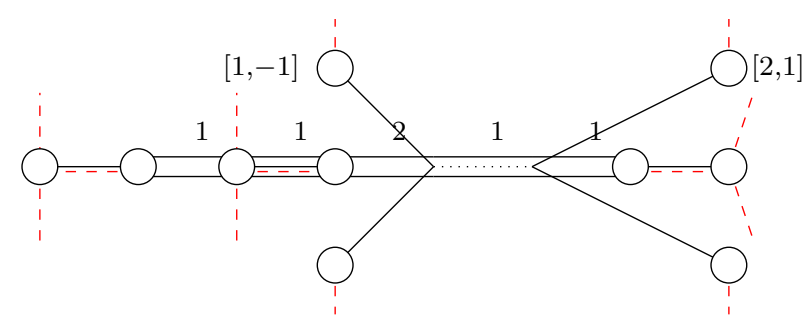

Inspecting $(2.36)$ shows that the $(1,-1) 5$-brane, in contrast to the $(2,1) 5$-brane, is not a supersymmetric configuration by itself and needs to be connected to a [1,0] 7-brane on the left-hand-side via another 5-brane.

Finite coupling. Configuration (2.36) can equivalently be described as

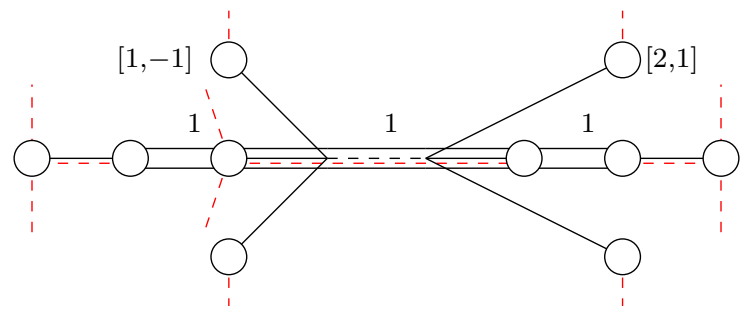

by transitioning the $(p, q)$ 5-brane outside the orientifold through the 7-branes and accounting for brane creation and annihilation. As above, the $(1,-1) 5$-branes between $\widetilde{\mathrm{O}}^{+}$and $\widetilde{\mathrm{O} 5}^{-}$are consistent configurations by themselves, but these are not Higgs branch degrees for freedom. The $(2,1) 5$-branes behave essentially like the $(1,-1)$ branes, because the half monodromy cut turns the $(2,1)$ close to the orientifold into a $(1,1)$ brane. In contrast, the collection of $(1,0) 5$-branes suspended between different 7-branes are all independent subwebs, representing non-trivial Higgs branch degrees of freedom. From (2.37), the magnetic quiver is read off to be

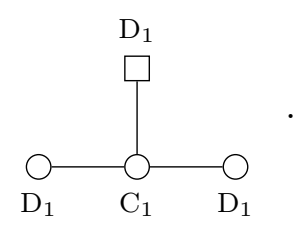

Straightforwardly computing the Coulomb branch dimension and global symmetry, see appendix A.3, yields

$$
\operatorname{dim}_{\mathbb{H}} \mathcal{C}(2.38)=3, \quad G=\mathrm{SO}(6)
$$

which matches the Higgs branch dimension as well as the non-abelian part of the global symmetry correctly. 
Infinite coupling. Proceeding to infinite coupling is realised by setting the distance between NS5 branes to zero. In other words, the $(1,1)$ and $(1,-1) 5$-branes become coincident on the $\mathrm{O} 5$ plane and the brane web is given by

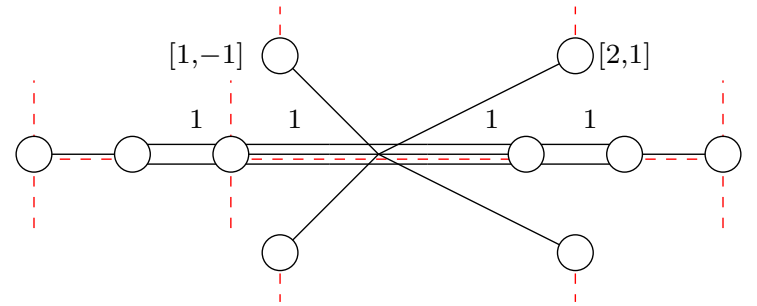

Crucially, this opens up a new Higgs branch direction, because the combination of the $(2,1)$ and $(1,-1) 5$-brane is a consistent subweb and free to move along the $x^{7,8,9}$ direction. As this subweb passes through the $\mathrm{O} 5$ plane, but is mapped to itself, the associated magnetic gauge multiplet is of $\mathrm{U}(1)$ type. Moreover, the intersection number with the central D5 brane is one, so there is one magnetic bi-fundamental hypermultiplet between the $\mathrm{U}(1)$ and the $\mathrm{C}_{1}$ node. As above, one needs to examine interplay of the $(1,-1)$ and $(2,1) 5$-branes closely. The intersection number of the 5 -branes evaluates to 3 , but becomes corrected by common 7-branes such that the generalised intersection number is 2 . Therefore, novel matter multiplets arise form the non-trivial generalised intersection number. To see what these might be, it is instructive to imagine the $(1,-1)$ and $(2,1) 5$-brane without the O5 plane. Then each 5-brane would lead to a U(1) magnetic vector multiplet and the intersection number dictates the number of copies of bifundamental matter that connects these magnetic gauge nodes. Including the orientifold then leads to an identification of these magnetic vector multiplets and it is suggestive that the two bifundamental magnetic hypermultiplets turn into one magnetic hypermultiplet of charge 2 for the magnetic $\mathrm{U}(1)$ gauge node.

Since the remainder of the web has not changed compared to the finite coupling case (2.37), the magnetic quiver for infinite coupling is read off to be

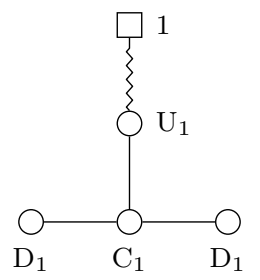

where the wiggly line denotes a hypermultiplet of charge 2 . The Coulomb branch dimension becomes

$$
\operatorname{dim}_{\mathbb{H}} \mathcal{C}(2.41)=4 .
$$

Again, the choice of magnetic gauge groups is expanded on in [80]; the subsequent Hilbert series analysis suggest that the Coulomb branch satisfies $\mathcal{C}(2.41)=\overline{\mathcal{O}}_{D_{4}}^{\min }$.

One may wonder why the identification in (2.41) is one hypermultiplet of charge two and not two hypermultiplets of charge one. The brane system for both looks the same. The answer to this is coming form the computation of the Higgs branch of (2.41) which should have dimension 1 and not 2 . 


\subsection{6 $E_{3}: \operatorname{Sp}(1)$ with 2 flavours}

Next, considering $\operatorname{Sp}(1)$ with $N_{f}=2$ flavours, the relevant 5 -brane web is given by

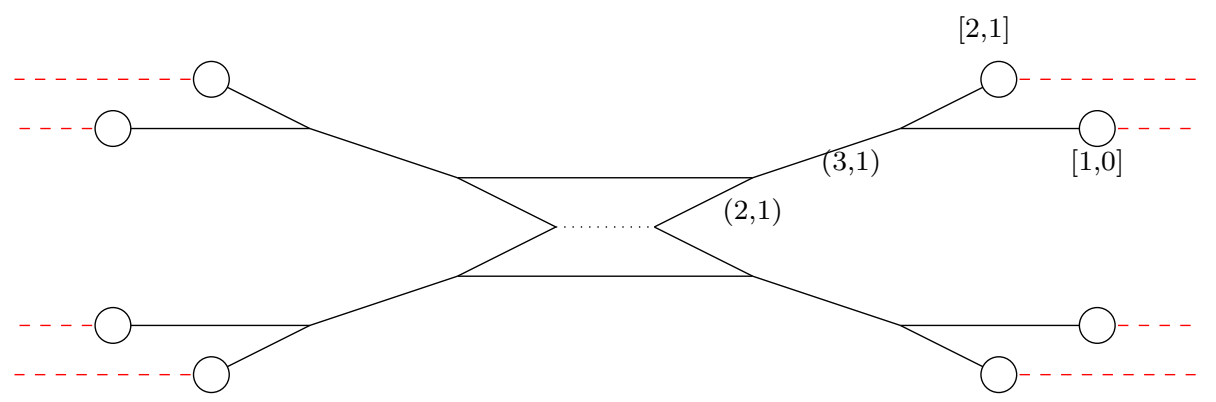

where the two flavours have been chosen symmetrically. Transitioning into the Higgs branch phase, the gauge and flavour D5s align with the O5 plane and the flavour half 7-branes are split along the orientifold. Then, the brane web becomes

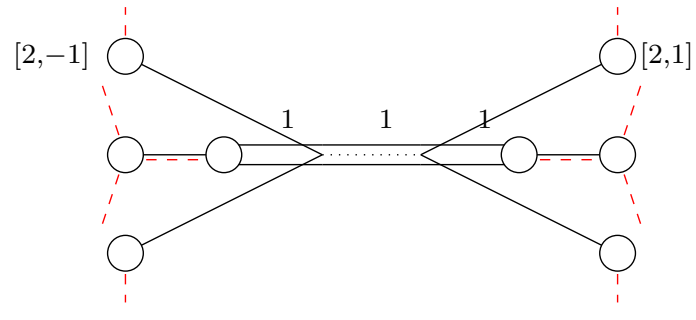

and one observes that the $(2, \pm 1) 5$-brane are already free configurations.

Finite coupling. To read off the magnetic quiver at finite coupling, the $(2, \pm 1) 5$-branes in (2.44) can stay where they are, as one can read a magnetic $\mathrm{O5}^{-}$plane with two magnetic flavours. Thus, the magnetic quiver associated to (2.44) is found to be

$$
\begin{gathered}
\mathrm{C}_{1} \\
\square \\
\square \\
\mathrm{D}_{1}
\end{gathered}
$$

which has a Coulomb branch of quaternionic dimension one. In fact, it is known that

$$
\mathcal{C}(2.45)=\overline{\mathcal{O}}_{\mathrm{D}_{2}}^{\min }
$$

As known from [94], the finite coupling Higgs branch is a union of two (isomorphic) cones. Correspondingly, one would expect two maximal inequivalent subdivision of the brane web (2.44), but the current observations show only one. 
Infinite coupling. To reach the infinite coupling phase, the $(2, \pm 1) 5$-branes in $(2.44)$ are made coincident along the orientifold such that the web becomes

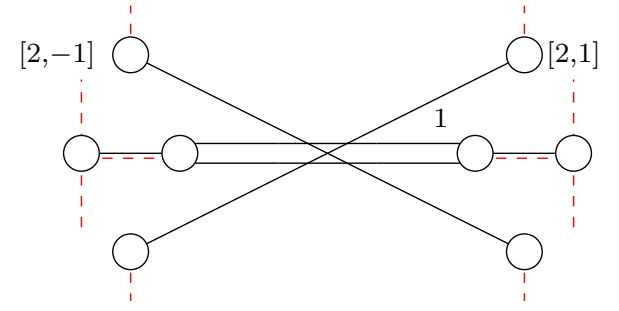

and one can proceed to read off the Higgs branch degrees of freedom. The subweb consisting of a $\mathrm{D} 5$ on top of an $\mathrm{O}^{-}$plane provides a $\mathrm{D}_{1}$ magnetic node. The subweb formed by the $(2, \pm 1)$ branes yields a $\mathrm{U}(1)$ node, that is connected by a bifundamental to the $\mathrm{D}_{1}$ node because the intersection number is 1 . In addition, the self-intersection of the $(2, \pm 1)$ branes needs be considered. The intersection number of the $(2, \pm 1) 5$-branes is four, but there are also common 7-branes to be take into account. In total, the generalised intersection number is 2, which then suggest that the $\mathrm{U}(1)$ magnetic gauge node needs to be supplemented by one magnetic hypermultiplet of charge 2 . Thus, the magnetic quiver is given by

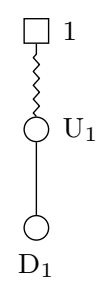

Notice that only a $U_{1} \subset C_{1}$ subalgebra of the $C_{1}$ flavour symmetry in (2.45) is gauged. The moduli space becomes

$$
\mathcal{C}(2.48)=\overline{\mathcal{O}}_{A_{2}}^{\min }
$$

which is also denoted as $a_{2}$.

It should be noted that the expectation is that there should be two maximal subdivisions [24, section 2.1] in order to reflect the existence of two cones $e_{3} \cong a_{2} \cup a_{1}[1,2]$. However, the 5-brane web (2.47) does not show any signs for a second subdivision.

\subsection{7 $E_{2}: \operatorname{Sp}(1)$ with 1 flavour}

Reducing the number of flavours to $N_{f}=1$, the 5 -brane web is given by

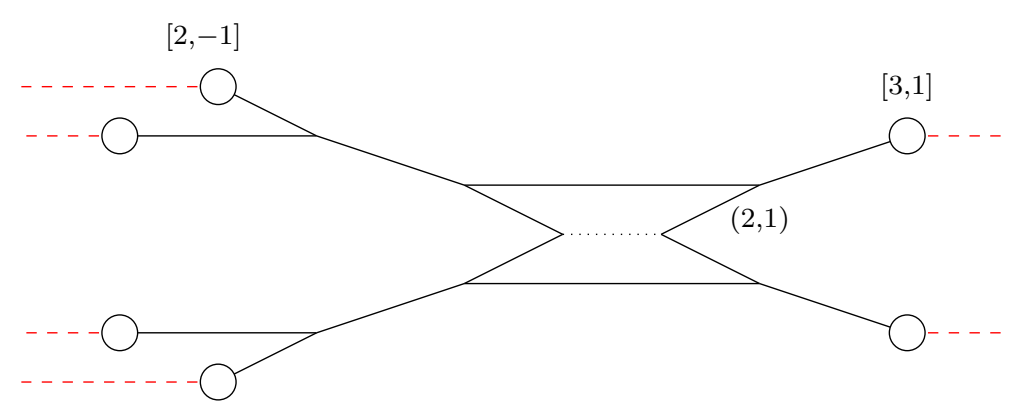


and one transitions to the Higgs branch phase by the same steps as above. Thus, one arrives at

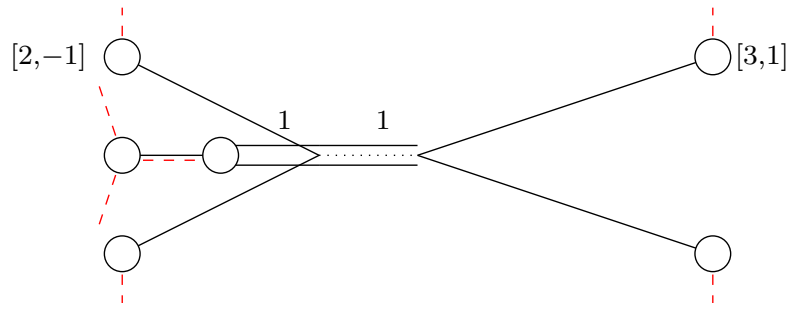

where the $(2,-1)$ brane is a free configuration. However, the $(3,1) 5$-brane is connected via a D5 brane to the 7-branes on the left-hand-side.

Finite coupling. Eliminating the non-dynamical branes via brane annihilation leads to a clean Higgs branch phase at finite coupling:

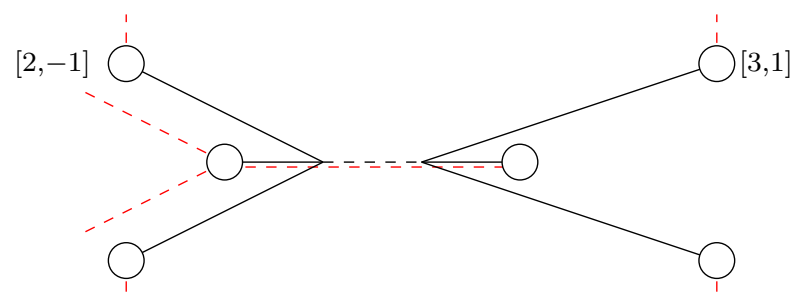

and one realises that there are no Higgs branch directions. Hence, the magnetic quiver is empty.

Infinite coupling. From (2.52) one takes the gauge coupling to infinity by making the $(2,-1)$ and $(3,1) 5$-brane coincident on the orientifold. The 5 -brane web is

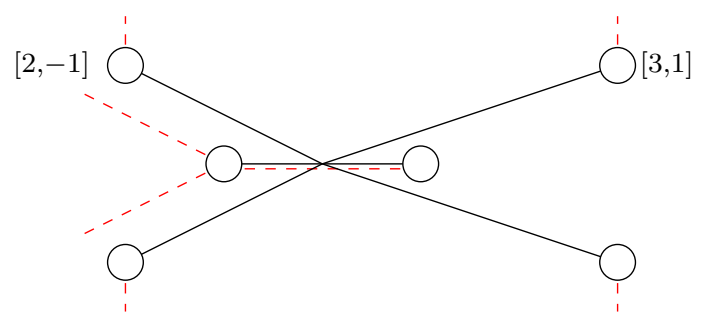

and one recognises a new Higgs branch direction. The subweb consisting of the $(2,-1)$ and $(3,1)$ brane yield a $\mathrm{U}(1)$ magnetic gauge group, and the self-intersection of 4 contributes two magnetic hypermultiplets of charge 2 . Therefore, the magnetic quiver becomes

$$
\xi_{\mathrm{U}_{1}}^{2}
$$

such that the Coulomb branch is

$$
\mathcal{C}(2.54)=\mathbb{C}^{2} / \mathbb{Z}_{2}
$$

which equals the $A_{1}$ part of the $e_{2} \cong A_{1} \cup \mathbb{Z}_{2}$ moduli space. The $\mathbb{Z}_{2}$ factor is due to a nilpotent operator in the infinite coupling Higgs branch chiral ring [57] and hence not detectable with current magnetic quiver methods. 


\subsection{8 $E_{1}$ : pure $\operatorname{Sp}(1)$ theory}

Now, consider pure $\operatorname{Sp}(1)$ gauge theory with brane web given by

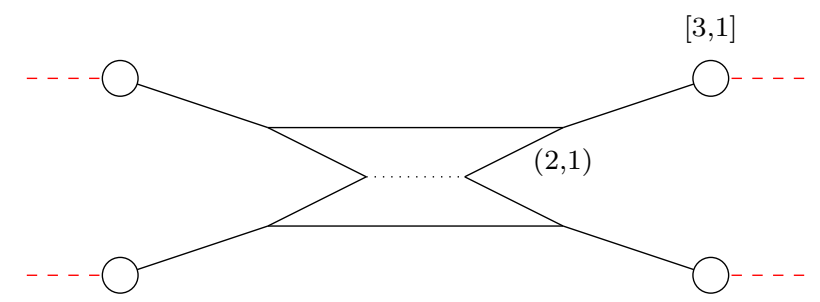

with no flavours.

Finite coupling. The Higgs branch phase is realised by aligning the D5 with the orientifold. Hence, the web becomes

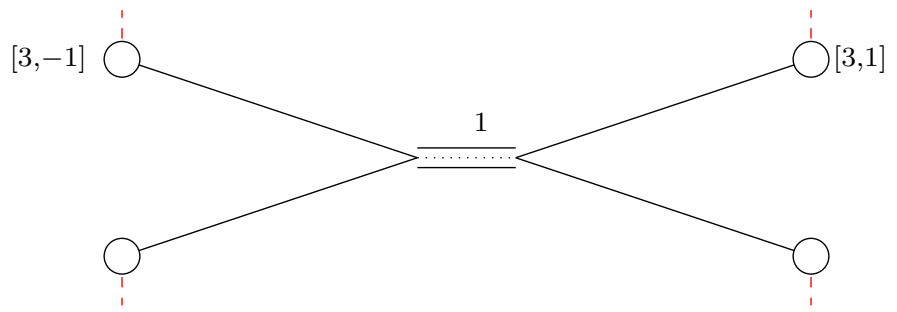

and shows that there are no Higgs branch directions at finite coupling. Consequently, the magnetic quiver is empty.

Infinite coupling. Transitioning (2.57) to infinite coupling, by making the $(3, \pm 1)$ branes coincident, the brane web reads

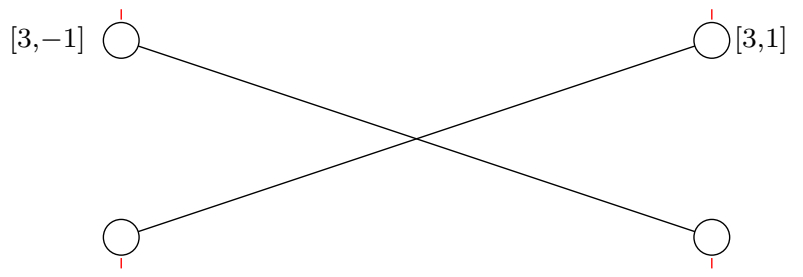

and one recognises one additional Higgs branch direction. The magnetic gauge group is a U(1), and the generalised self-intersection of 4 between the $(3 \pm 1)$ 5-branes suggest that there are two magnetic hypermultiplets of charge 2 . The associated magnetic quiver becomes

$$
\left\{\begin{array}{l}
2 \\
\mathrm{U}_{1}
\end{array}\right.
$$

and the moduli space is

$$
\mathcal{C}(2.59)=\mathbb{C}^{2} / \mathbb{Z}_{2}
$$

i.e. the $e_{1} \cong A_{1}$ space; in agreement with $[24,57]$. 
The $\widetilde{\boldsymbol{E}}_{\mathbf{1}}$ theory. In [22] an O5 construction for the $\widetilde{E}_{1}$ theory, whose Higgs branch is known to be a single point [4], was proposed; however, this is not elaborated here and the reader is referred to the discussion in [22].

\subsection{9 $\quad E_{0}$ theory}

Finally, to conclude this subsection, it is natural to introduce an $E_{0}$ theory. It does not admit a gauge theory description, as the number of flavours would need to be negative. However, as shown in tables 1-4, higher rank theories allow to define $E_{n}$ families for arbitrary $-\infty<n \leq 8$.

The family of interest here is $E_{4-2 l}$, specifically for $l=2$. Brane systems, magnetic quivers and Hasse diagrams are derived in full generality in the coming sections for rank $k$ satisfying $k \geq l$; for $k=1$ one can attempt an analytic continuation. As shown in table 3 , the dimension of the Higgs branch at infinite coupling is 0, consistent with the expectation that the $E_{0}$ theory has a Higgs branch which is a single point [4]. Correspondingly the Hasse diagram is reduced to a single trivial leaf, which is a point.

\subsection{Rules for magnetic quivers}

After establishing the validity of the magnetic quiver proposal by using magnetic orientifolds of table 7 , one has to formulate the proposal for a generic 5-brane web in the presence of O5 planes. Building on section 2.3, the magnetic quivers for a given 5-brane web in the Higgs branch phase are derived as follows.

Consider a 5-brane web with O5 planes in which each external half $(p, q) 5$-brane is terminated on a half $[p, q]$ 7-brane.

Conjecture 1 (Magnetic Quiver) The Higgs branch phase is realised when all the flavour and all gauge D5 branes are coincident with the 05 plane, such that the resulting physical [1,0] 7-branes are split along the orientifold. Then, for each inequivalent maximal subdivision into subwebs suspended between 7-branes, which is compatible with supersymmetry, the associated magnetic quiver can be derived by the following set of rules:

- Gauge nodes: a stack of $k$ identical subwebs which are free to move in the $x^{7,8,9}$ direction contribute a magnetic gauge multiplet.

(a) A stack of D5s, on top of an O5 plane, the magnetic gauge algebra is the one naturally associate to the magnetic orientifold.

(b) A stack of NS5s, on top of an O5 plane, the magnetic gauge algebra is determined by, firstly, transitioning to the magnetic orientifold of table 7 and, secondly, using the corresponding flavour algebra of table 6 as magnetic gauge algebra.

(c) A stack of any other kind of subwebs contributes a $\mathrm{U}(k)$ magnetic gauge multiplet. In addition, observation 1 may apply.

- Flavour nodes: the 5-branes that are allowed by supersymmetry, but are not free to move along the $x^{7,8,9}$ direction, only contribute flavour nodes to the magnetic quiver. The type of flavour is dictated by the magnetic orientifold. Most notably, a $(1, \pm 1)$ 5-brane between a $\widetilde{O 5}^{+}$and $\widetilde{O 5}^{-}$plane contributes a $B_{0}$ magnetic flavour, denoting a single half-hypermultiplet. 
- Matter: two non-identical subwebs are linked by a number of bi-fundamental magnetic hypermultiplets, determined by the generalised intersection number.

In addition, there are infinite coupling configurations that involve $(p, q)$ 5-branes which are neither D5 nor NS5 branes. The corresponding contributions to magnetic quivers are observed to be as follows:

Observation 1 (Charged matter) For a pair of $(r, \pm 1)$ 5-branes on a O5- plane, the stable intersection between of the two mirror half 5-branes is $2 r$, while the common 7-branes contribute $a-2$ such that the generalised intersection number is $2 r-2$. The $\mathrm{U}(1)$ node is supplemented by $\frac{2 r-2}{2}$ charge 2 hypermultiplets and the magnetic quiver reads

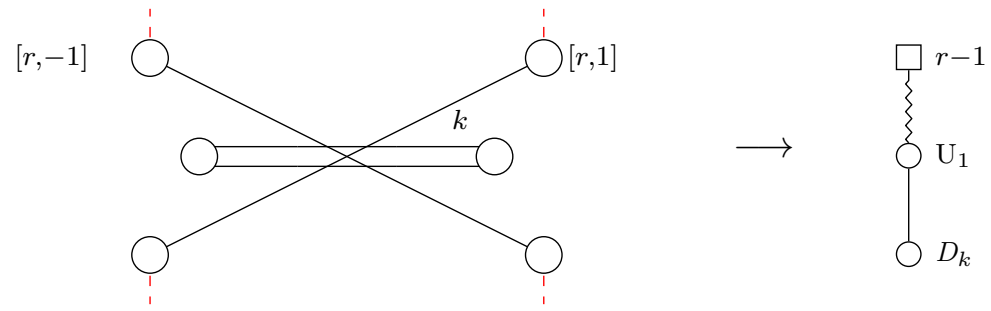

Analogously, for a pair of $(r,-1)$ and $(r+1,1)$ 5-branes on a $\widetilde{O 5}^{-}$plane, the generalised intersection number of the two mirror half 5-branes is $2 r-1$. The $\widetilde{O 5}^{-}$plane is suspected to additionally modify the intersection number by +1 due to the stuck half D5. The U(1) node has $r$ charge 2 magnetic hypermultiplets such that the contribution to the magnetic quiver then becomes

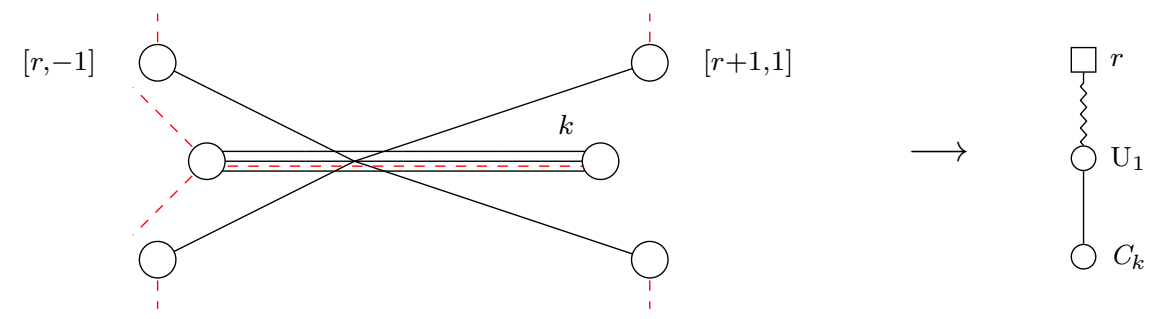

As a comment, contrary to 5-brane web without orientifolds [24], the examples considered in this paper do not show any signs of multiple inequivalent subdivisions for a given 5 -brane web. However, the possibility is covered by conjecture 1 .

\section{$3 \quad$ Single $\mathrm{Sp}(\mathrm{k})$ gauge group}

Having developed the magnetic quiver proposal for 5-brane webs with O5 planes, this section focuses on brane constructions for $\operatorname{Sp}(k)$ gauge theories with fundamental hypermultiplets. To begin with, known facts from field theory and 5-brane webs are collected and contrasted.

Field theory. For a $5 \mathrm{~d} \mathcal{N}=1 \mathrm{Sp}(k)$ gauge theory with $N_{f}$ fundamental flavours, a non-trivial interacting fixed point has been argued to exist for $N_{f} \leq 2 k+4$ in [3, section 4]. 
Suppose $N_{f} \geq 2 k$, considering the finite coupling Higgs branch yields

$$
\operatorname{dim}_{\mathbb{H}} \mathcal{H}=\frac{1}{2} \cdot 2 N_{f} \cdot 2 k-\operatorname{dim} \operatorname{Sp}(k)=k\left(2 N_{f}-2 k-1\right) .
$$

For $N_{f} \geq 2 k$, complete Higgsing is possible and, according to [94], the expectation for the finite coupling Higgs branch is as follows:

$$
\begin{aligned}
\mathcal{H} & =\left\{M \in \operatorname{Mat}_{2 N_{f} \times 2 N_{f}} \mid M+M^{T}=0, M^{2}=0, \operatorname{rank}(M) \leq 2 k\right\} \\
& \left.=\overline{\mathcal{O}}_{\mathrm{D}_{N_{f}}}^{[2 k}, 1^{2 N_{f}-4 k}\right]
\end{aligned}
$$

- $N_{f}>2 k$ : the Higgs branch is a single cone which is a nilpotent orbit closure of $\mathfrak{s o}\left(2 N_{f}\right)$.

- $N_{f}=2 k$ : the Higgs branch is a union of two cones.

For $N_{f}<2 k$, only partial Higgsing is possible and the claim is as follows:

- $N_{f}$ even: partial Higgsing to

$$
\operatorname{Sp}(k) \text { with } N_{f} \text { flavours } \longrightarrow \text { pure } \operatorname{Sp}\left(k-\frac{N_{f}}{2}\right)
$$

such that the Higgs branch dimension becomes

$$
\begin{aligned}
\operatorname{dim}_{\mathbb{H}} \mathcal{H} & =\frac{1}{2} 2 N_{f} \cdot 2 k-\left(\operatorname{dim} \operatorname{Sp}(k)-\operatorname{dim} \operatorname{Sp}\left(k-\frac{N_{f}}{2}\right)\right) \\
& =\frac{1}{2} N_{f}\left(N_{f}-1\right) .
\end{aligned}
$$

Again, the Higgs branch is a union of two cones

$$
\mathcal{H}=\overline{\mathcal{O}}_{\mathrm{D}_{N_{f}}^{\left[2_{f}\right.}}^{2^{N_{f}}} .
$$

- $N_{f}$ odd: partial Higgsing to

$$
\operatorname{Sp}(k) \text { with } N_{f} \text { flavours } \longrightarrow \operatorname{Sp}\left(k-\frac{N_{f}-1}{2}\right) \text { with } 1 \text { flavour }
$$

and the Higgs branch dimension is computed to be

$$
\begin{aligned}
\operatorname{dim}_{\mathbb{H}} \mathcal{H} & =\frac{1}{2}\left(2 N_{f} \cdot 2 k-2 \cdot\left(k-\frac{N_{f}-1}{2}\right)\right)-\left(\operatorname{dim} \operatorname{Sp}(k)-\operatorname{dim} \operatorname{Sp}\left(k-\frac{N_{f}-1}{2}\right)\right) \\
& =\frac{1}{2} N_{f}\left(N_{f}-1\right) .
\end{aligned}
$$

In fact, the Higgs branch equals

$$
\mathcal{H}=\overline{\mathcal{O}}_{\mathrm{D}_{N_{f}}}^{\left[2^{N_{f}-1}, 1^{2}\right]} .
$$

5-brane web. The requirement that the external 5-branes do not intersect, which would introduce additional massless degrees of freedom, leads to the constraint [8, section 3.4] that $N_{f} \leq 2 k+4$. It has been argued in [9, section 2] that the case $N_{f}=2 k+5$ also gives rise to a consistent $5 \mathrm{~d}$ theory, which for $k=1$ yields the familiar $E_{8}$ theory. 
The change in Higgs branch dimension when approaching the fixed point has been studied in [10] and can be summarised as follows:

- $0 \leq N_{f} \leq 2 k$ : the number of D5s in between the branes with non-zero NS charge is larger than on the outside. Then exactly one extra dimension is gained by detaching the 5-branes from the $\mathrm{O} 5$ plane after collapsing the gauge 5-branes.

- $2 k+1 \leq N_{f} \leq 2 k+3$ : the number of D5s in between the branes with non-zero NS charge is smaller compared to the outside. Then at infinite coupling, there exists one extra dimension due to the $(p, q)$ 5-branes with $q>0$. Moreover, there exist further additional Higgs branch directions originating from the D5s that can reconnect once the gauge branes collapsed.

- $N_{f}=2 k+4$ : there are new Higgs branch directions due to D5s that reconnect once the gauge branes collapsed. Moreover, one extra dimension is gained by detaching the 5-branes from the O5 plane after collapsing the gauge 5-branes; the external NS5 branes are parallel and an additional direction becomes accessible by breaking one of them on a 7-brane.

- $N_{f}=2 k+5$ : the external 5-branes intersect and the resolution thereof yields several additional directions. One extra dimension is gained by detaching the 5-branes from the O5 plane after collapsing the gauge 5-branes; while several other directions open up due to D5 branes that can reconnect once the gauge branes collapsed.

In addition, $[9,35]$ investigated the potential enhancement of the classical $\mathrm{SO}\left(2 N_{f}\right) \times \mathrm{U}(1)_{I}$ global symmetry and the claim is that

- $N_{f}<2 k+4$ : no enhancement.

- $N_{f}=2 k+4$ : the $\mathrm{U}(1)_{I}$ factor enhances to a $\mathrm{SU}(2)$.

- $N_{f}=2 k+5$ : the symmetry enhances as $\mathrm{SO}(4 k+10) \times \mathrm{U}(1)_{I} \rightarrow \mathrm{SO}(4 k+12)$.

\section{1 $\mathrm{Sp}(\mathrm{k})$ with fundamental flavours and $\mathrm{O5}^{+}$}

The construction of the 5 -brane web for a $5 \mathrm{~d} \mathcal{N}=1 \mathrm{Sp}(k)$ gauge theory with $N_{f}$ fundamental flavours follows [10]. In detail, the brane web for $N_{R}$ flavour branes on the right-handside and $N_{L}$ flavour brane on the left-hand-side starts from the Coulomb branch phase with identical masses given by

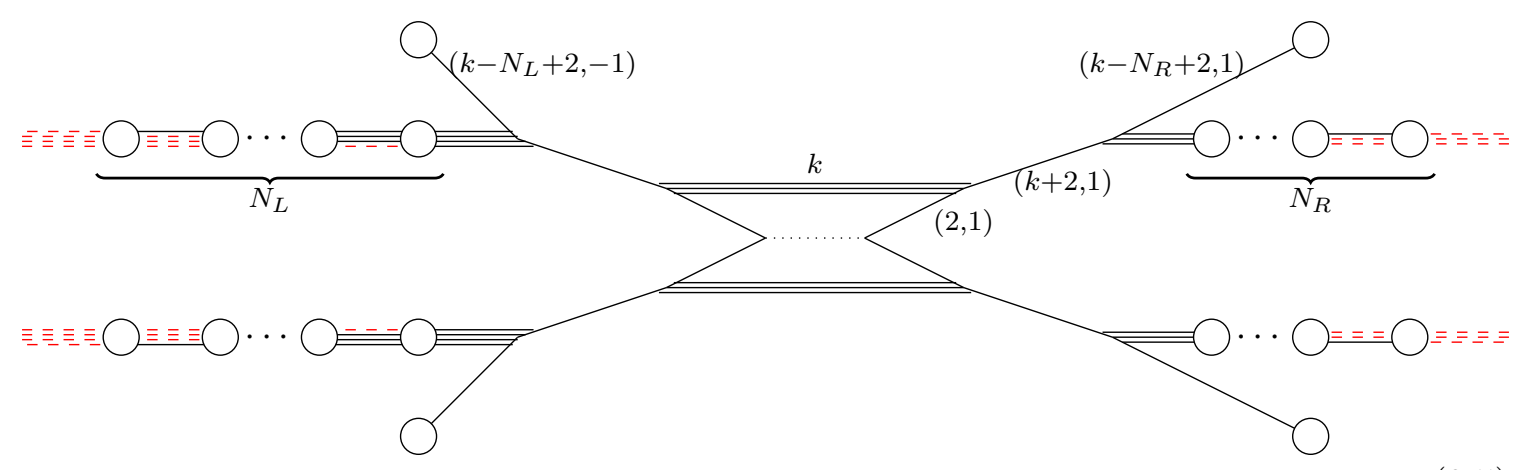

such that the transition to the Higgs branch phase is again achieved in several steps: firstly, the flavour half $[1,0] 7$-branes as well as the gauge $(1,0) 5$-branes move towards the O5 plane 
such that the half 7-branes merge with their mirror images. Secondly, the newly formed physical 7-branes can be split along the orientifold such that the 5-brane web becomes

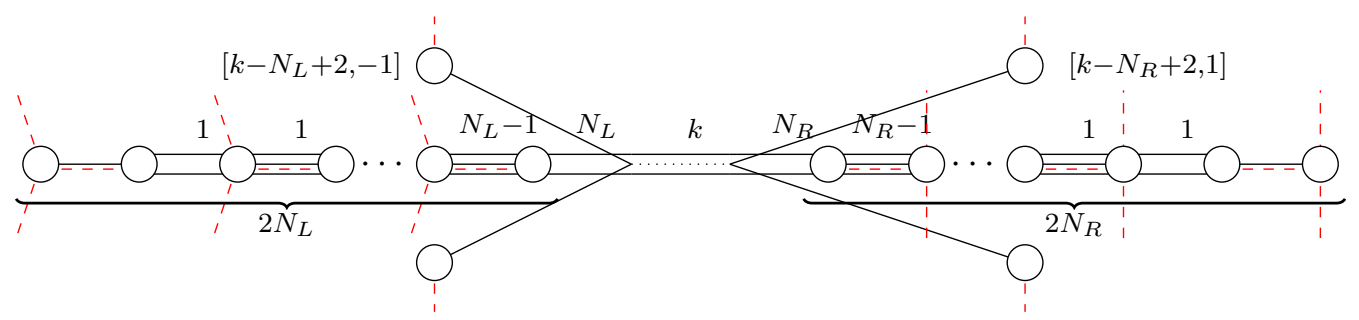

using the O5 plane notation of table 6. Without loss of generality, it is sufficient to consider two cases

(i) $N_{f}$ even: $N_{L}=N_{R}=\frac{N_{f}}{2}$,

(ii) $N_{f}$ odd: $N_{L}=N_{R}+1=\frac{N_{f}+1}{2}$.

This is because any other configuration can be transitioned into one of the two cases by moving sufficiently many flavour branes from one side to the other, due to brane annihilation and creation.

\subsubsection{Finite coupling}

$\boldsymbol{N}_{\boldsymbol{f}}$ even. For $N_{L}=N_{R}$ the brane configuration (3.6) simplifies to

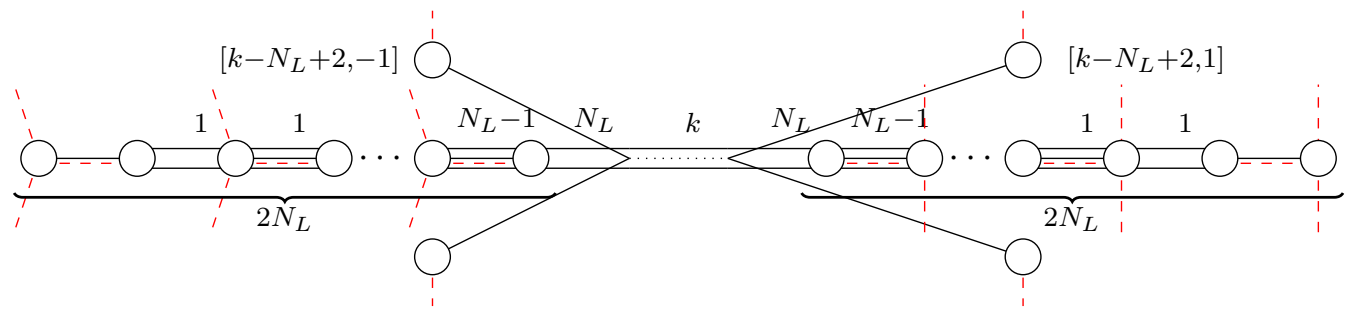

If $N_{L} \leq k$ or, equivalently, $N_{f} \leq 2 k$ then only $N_{L} 5$-branes in the central segment of $k$ physical $(1,0) 5$-branes are dynamical. In particular, $k-N_{L}$ of the central 5 -branes remain suspended between NS5 branes such that there is a residual $\operatorname{Sp}\left(k-\frac{N_{f}}{2}\right)$ gauge group left. For the 5-branes that contribute to the Higgs branch, the associated magnetic quiver reads

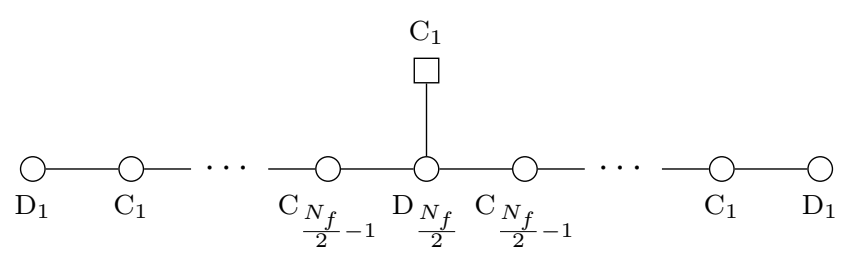

and one computes

$$
\operatorname{dim}_{\mathbb{H}} \mathcal{C}(3.8)=\frac{1}{2} N_{f}\left(N_{f}-1\right), \quad G=\operatorname{SO}\left(2 N_{f}\right)
$$

because all gauge nodes are balanced, cf. appendix A.3. The nilpotent orbit $\mathcal{O}_{\mathrm{D}_{N_{f}}}^{\left[2^{N_{f}}\right]}=$ $\mathcal{O}_{\mathrm{D}_{N_{f}}}^{\left[2^{N_{f}}\right] I} \cup \mathcal{O}_{D_{N_{f}}}^{\left[2^{N_{f}}\right] I I}$ of $\mathfrak{s o}\left(2 N_{f}\right)$ is a union of two nilpotent orbits [95], the Coulomb branch 
of (3.8) is the closure of one of these orbits. In particular, the properties (3.9) agree with the expectation (3.3) and the brane web (3.7) displays the residual gauge theory explicitly. As in the case of $\operatorname{Sp}(1)$ only one cone can be identified from the web, however a direct computation of the hyper-Kähler quotient shows, that there are indeed two cones. See section 4.5 for more on the two cone problem.

If $N_{L}>k$ or, equivalently, $N_{f} \geq 2 k+2$ then all of the $k$ central branes are dynamical Higgs branch degrees of freedom. The $\left(k-N_{L}+1, \pm 1\right) 5$-branes are connected to 7branes via $N_{L}-k$ branes, which can be annihilated when moving the $\left(k-N_{L}+1, \pm 1\right)$ 5-branes through sufficiently many 7-branes, as discussed above. Hence, the magnetic quiver becomes

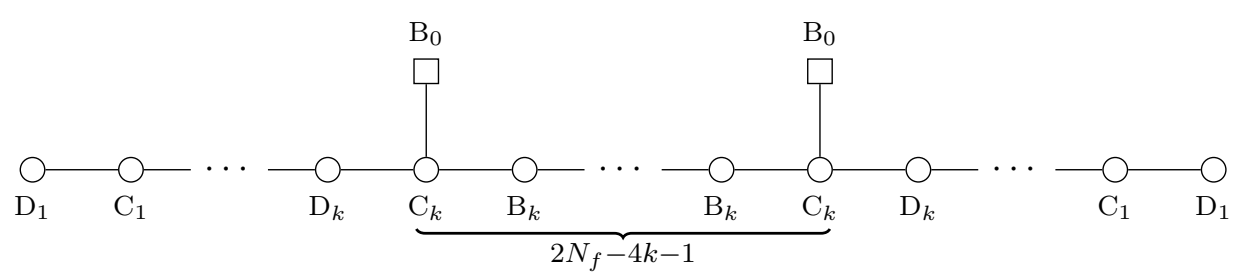

and one verifies

$$
\operatorname{dim}_{\mathbb{H}} \mathcal{C}(3.10)=k\left(2 N_{f}-2 k-1\right), \quad G=\mathrm{SO}\left(2 N_{f}\right)
$$

because all gauge nodes are balanced, cf. appendix A.3. Moreover, the Coulomb branch geometry is known to be

$$
\mathcal{C}(3.10)=\overline{\mathcal{O}}_{\mathrm{D}_{N_{f}}}^{\left[2^{2 k}, 1^{2 N_{f}-4 k}\right]}
$$

i.e. a nilpotent orbit closure for $\mathfrak{s o}\left(2 N_{f}\right)$. Consequently, the properties (3.11) correctly reproduce the finite coupling Higgs branch.

$\boldsymbol{N}_{\boldsymbol{f}}$ odd. For $N_{L}=N_{R}+1$ the brane configuration (3.6) specialises to

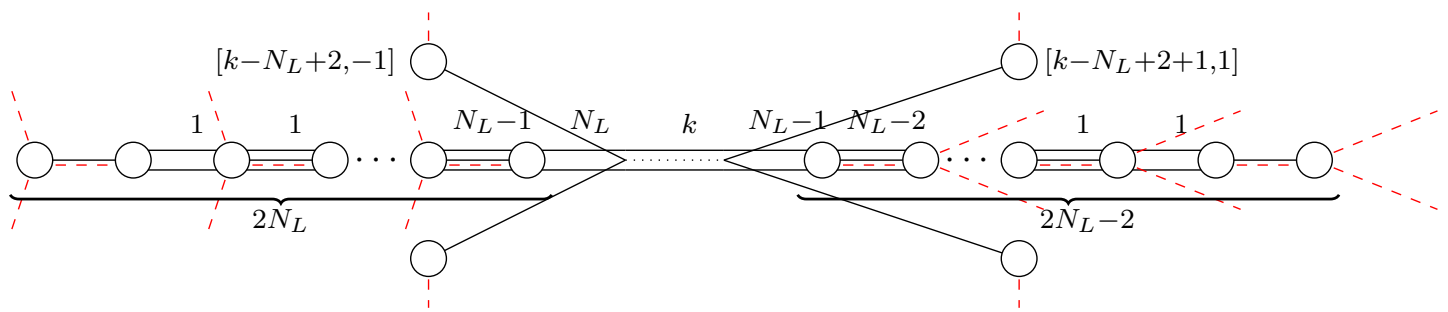

Regardless of the value of $N_{f}$, after moving both, the $\left(k-N_{L}+2-1,1\right)$ as well as the $\left(k-N_{L}+2,-1\right) 5$-brane through the first 7-brane on the left-hand side, the brane configuration becomes

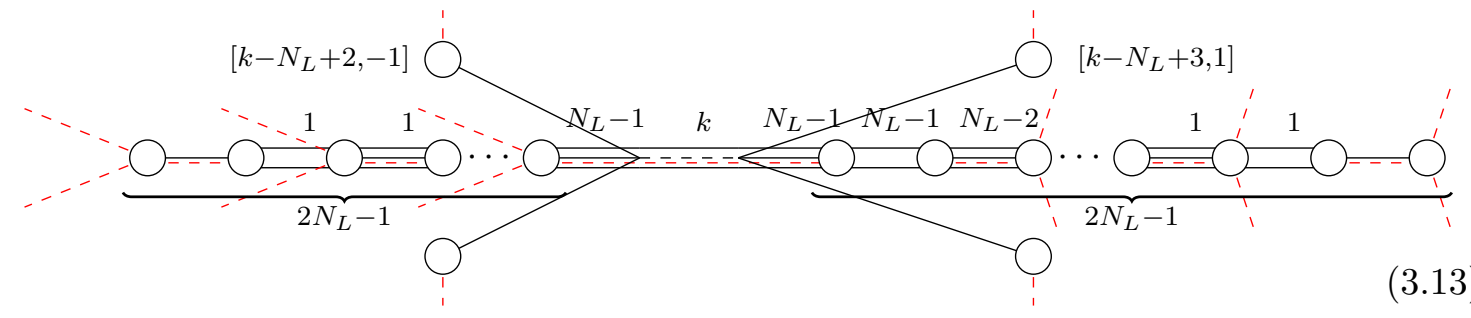


If $N_{L}-1 \leq k$ or, equivalently, $N_{f} \leq 2 k+1$ only $\frac{N_{f}-1}{2} k$ of the $k$ central 5 -branes are dynamical Higgs branch degrees of freedom. Hence, $k-\frac{N_{f}-1}{2} 5$-branes remain suspended between NS5 branes and indicate a residual Sp $\left(k-\frac{N_{f}-1}{2}\right)^{2}$ gauge group. In addition, the half 7-branes closest to the NS5 branes contribute a single flavour to the residual gauge group. From the remaining 5-branes that contribute to the Higgs branch one reads off the magnetic quiver as follows

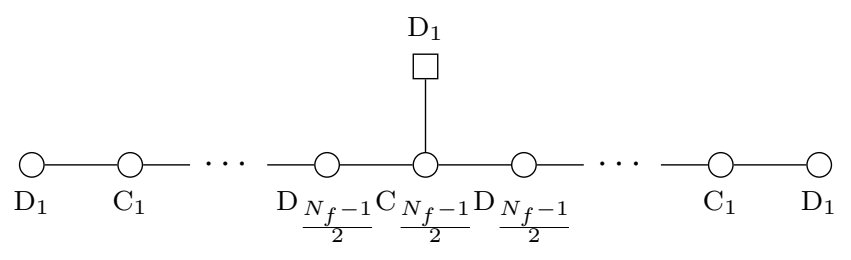

and one can compute

$$
\operatorname{dim}_{\mathbb{H}} \mathcal{C}(3.14)=\frac{1}{2} N_{f}\left(N_{f}-1\right), \quad G=\mathrm{SO}\left(2 N_{f}\right)
$$

because all gauge nodes are balanced, cf. appendix A.3. The Coulomb branch of (3.14) is the closure of the nilpotent orbit $\left[2^{N_{f}-1}, 1^{2}\right]$ of $\mathfrak{s o}\left(2 N_{f}\right)$. As a consequence, the properties (3.15) agree with the expectation (3.4) and the brane web displays the residual gauge theory explicitly.

On the other hand, if $N_{L}-1>k$ or, equivalently, $N_{f}>2 k+1$ then all of the $k$ central 5 -branes are Higgs branch degrees of freedom. As dictated by the S-rule, the $\left(k-N_{L}+2,-1\right)$ and $\left(k-N_{L}+3,1\right) 5$-branes are connected to 7 -branes via sufficiently many $(1,0) 5$-branes, which can be annihilated by moving the non-flavour 5-branes through 7-branes. As a result, one finds the following magnetic quiver

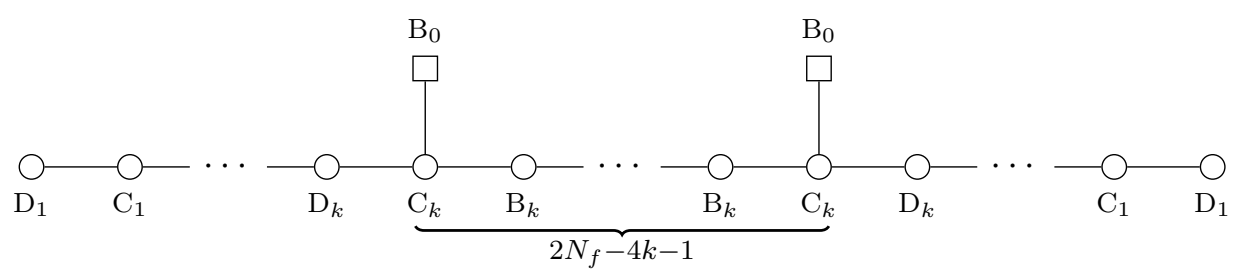

which is the same as for $N_{f}$ even and $N_{f} \geq 2 k+1$. A computation shows

$$
\operatorname{dim}_{\mathbb{H}} \mathcal{C}(3.16)=k\left(2 N_{f}-2 k-1\right), \quad G=\mathrm{SO}\left(2 N_{f}\right)
$$

because all gauge nodes are balanced, cf. appendix A.3. In fact

$$
\mathcal{C}(3.16)=\overline{\mathcal{O}}_{\mathrm{D}_{N_{f}}}^{\left[2^{2 k}, 1^{2 N_{f}-4 k}\right]},
$$

which is a nilpotent orbit closure of $\mathfrak{s o}\left(2 N_{f}\right)$. Therefore, the properties (3.17) match the finite coupling Higgs branch. 


\subsubsection{Infinite coupling}

$N_{f}$ even. For $N_{L}=N_{R}$ the 5 -brane web at infinite coupling has to be considered for two distinct cases. Firstly, if $N_{L}<k+2$ or, equivalently, $N_{f}<2 k+4$, the web becomes

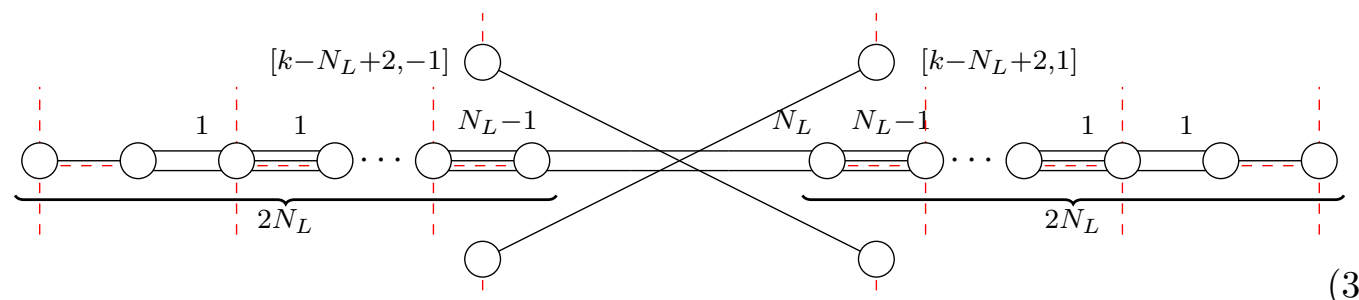

and the magnetic quiver is read off to be

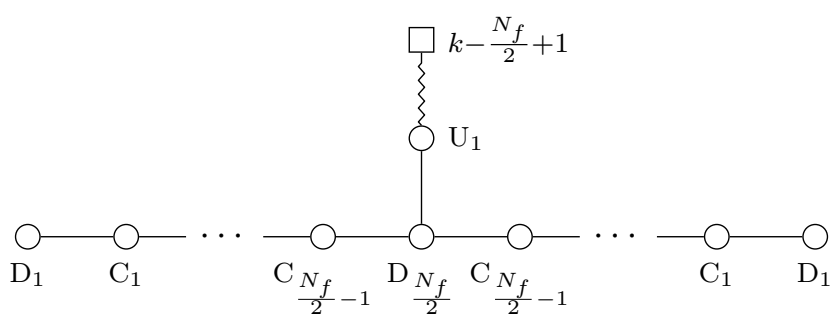

and one verifies

$$
\operatorname{dim}_{\mathbb{H}} \mathcal{C}(3.19)=\frac{N_{f}\left(N_{f}-1\right)}{2}+1 .
$$

The charge two magnetic hypermultiplets are expected from the self-intersection of the $\left(k-N_{L}+2, \pm 1\right) 5$-branes, as detailed in observation 1. Moreover, one notices that the family of magnetic quivers (3.19) contains the $E_{5}$ case (2.33), the $E_{3}$ case (2.48) and the $E_{1}$ case (2.59).

Secondly, if $N_{L}=k+2$ or, equivalently, $N_{f}=2 k+4$, the web at infinite coupling reads

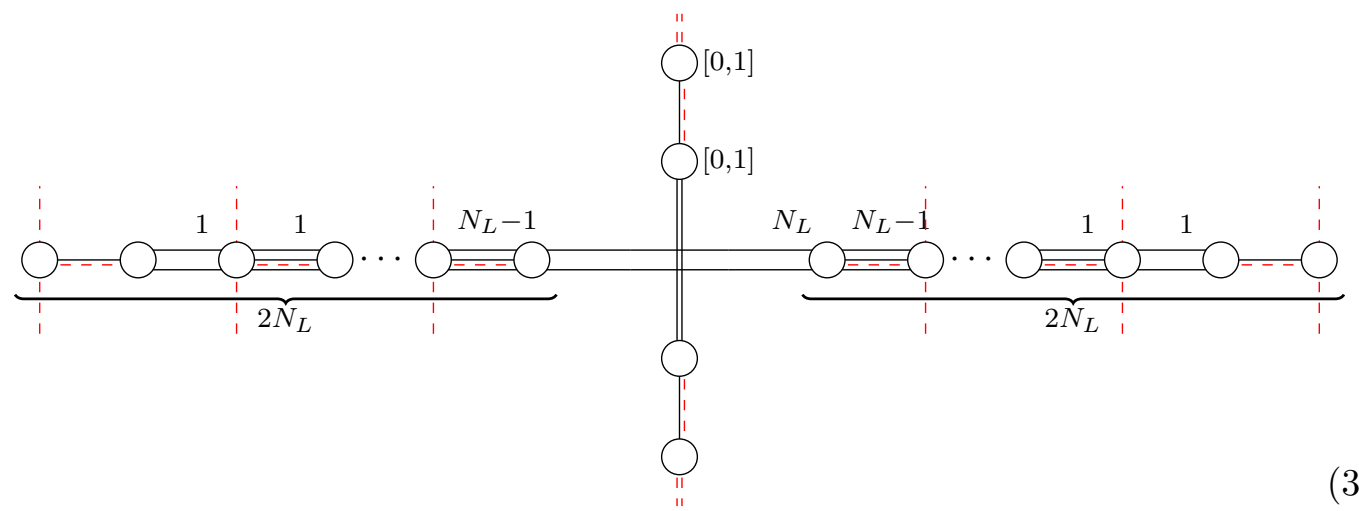

such that the magnetic quiver is

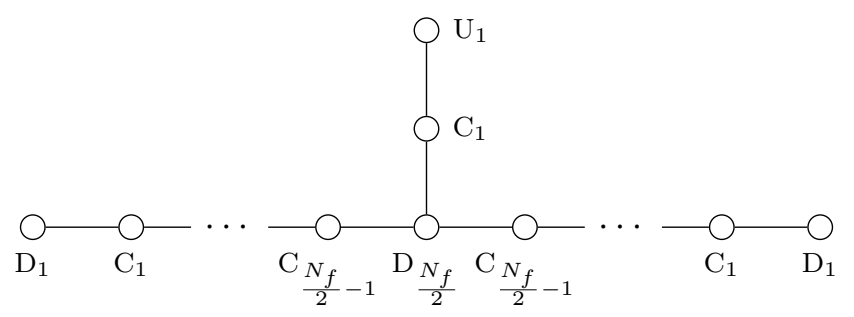


and the dimension is

$$
\operatorname{dim}_{\mathbb{H}} \mathcal{C}(3.22)=\frac{N_{f}\left(N_{f}-1\right)}{2}+2 .
$$

One observes that this family contains the $E_{7}$ case (2.17).

$\boldsymbol{N}_{\boldsymbol{f}}$ odd. For $N_{L}=N_{R}+1$ there exists two distinct cases for the brane web at infinite coupling. Firstly, for $N_{L}<k+3$ or, equivalently, $N_{f}<2 k+5$ the finite coupling 5-brane web (3.12) can be used to make the $\left(k-N_{L}+2,-1\right)$ and $\left(k-N_{L}+3,1\right) 5$-brane become coincident on the O5 plane. Doing so results in

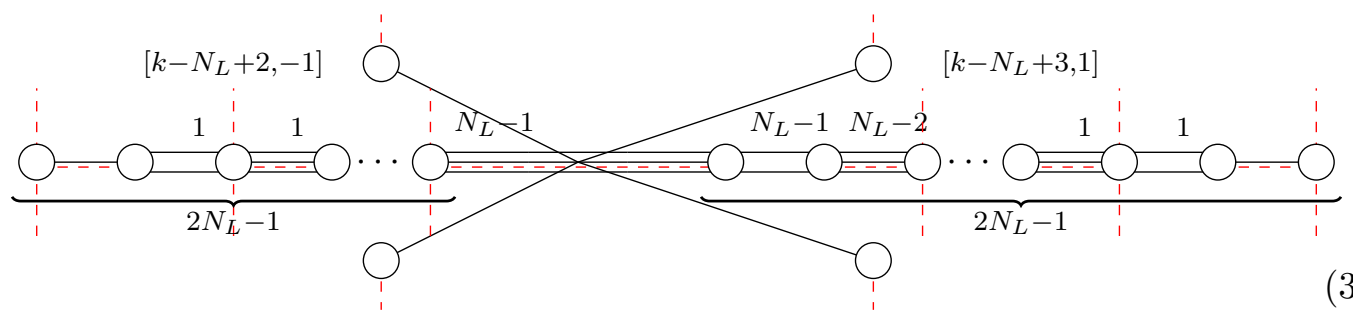

such that the magnetic quiver can be read off

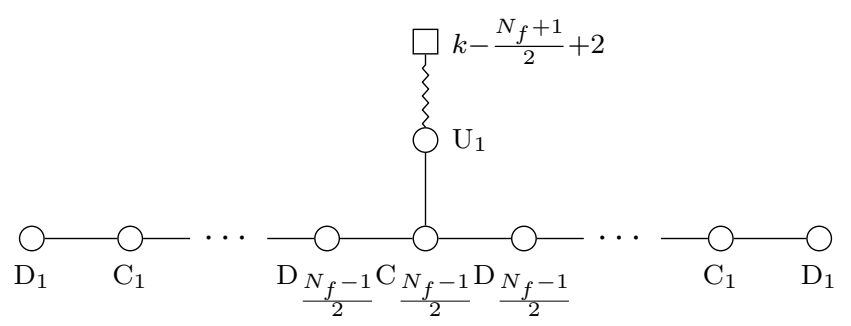

and the Coulomb branch dimension is

$$
\operatorname{dim}_{\mathbb{H}} \mathcal{C}(3.25)=\frac{N_{f}\left(N_{f}-1\right)}{2}+1 .
$$

The charge two magnetic hypermultiplet is an expected consequence from the intersection of the $\left(k-N_{L}+2,-1\right)$ and $\left(k-N_{L}+3,1\right) 5$-brane, as suggested in observation 1 . One notices that the family (3.25) of quivers contains the $E_{6}$ case (2.25), the $E_{4}$ case (2.41) and the $E_{2}$ case (2.54).

Secondly, for $N_{L}=k+3$ or, equivalently, $N_{f}=2 k+5$ the finite coupling brane web can be written as

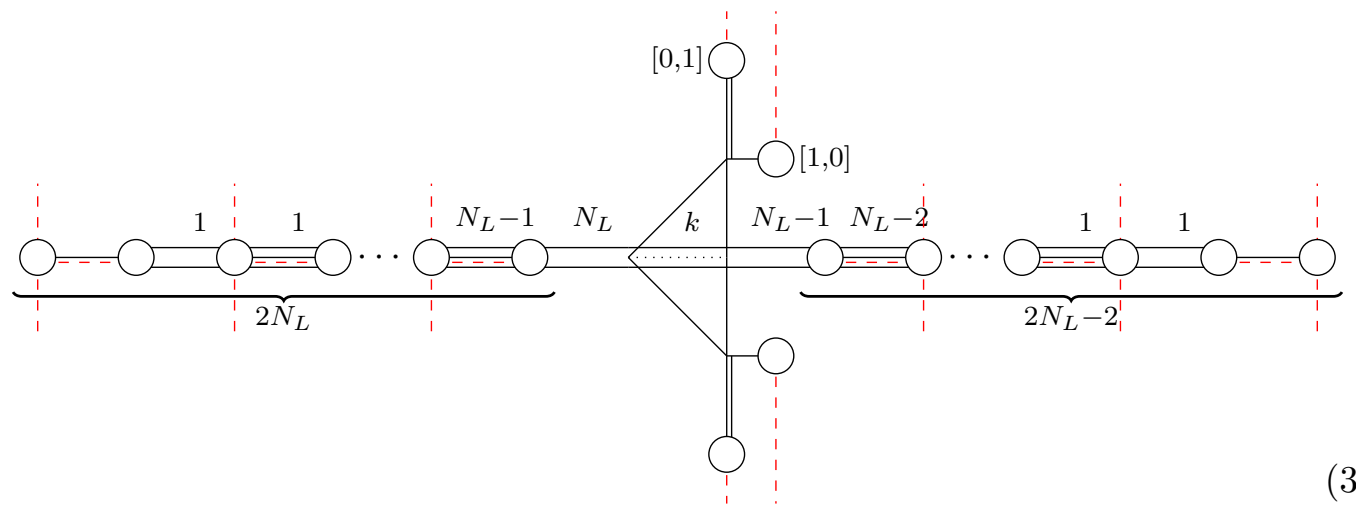


such that the infinite coupling configuration is reached once the $[1,0] 7$-brane moves onto the O5 plane, and the physical 7-brane splits along the orientifold. In detail, the brane web becomes

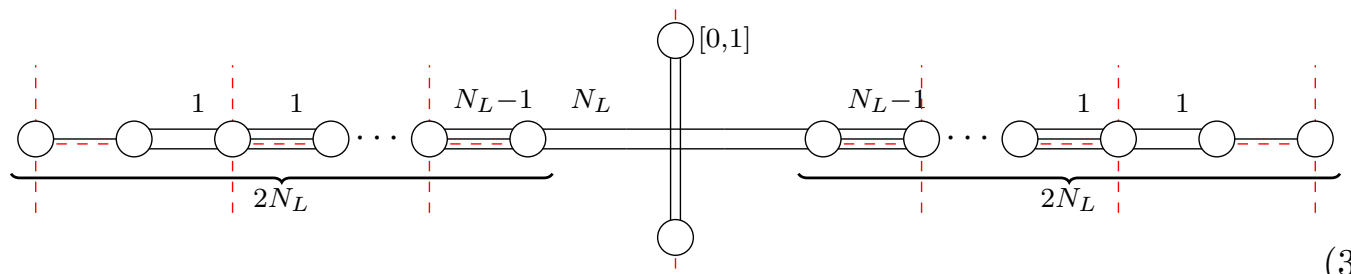

and the magnetic quiver is straightforwardly read off to be

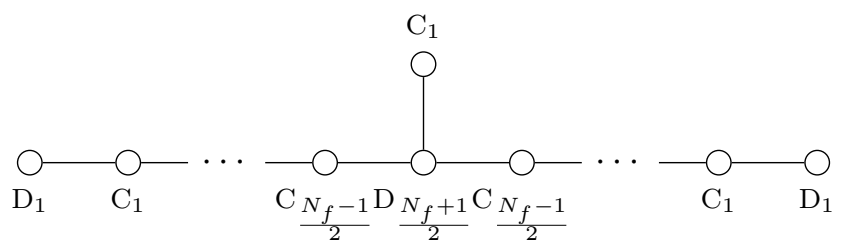

and

$$
\operatorname{dim}_{\mathbb{H}} \mathcal{C}(3.29)=\frac{N_{f}\left(N_{f}+1\right)}{2}+1
$$

One notices that this family contains the $E_{8}$ case (2.9).

\section{2 $\mathrm{Sp}(\mathrm{k})$ with fundamental flavours and $\widetilde{\mathrm{O5}}^{+}$}

Since an $\widetilde{\mathrm{O} 5}^{+}$plane does also give rise to symplectic low-energy gauge theories, it is tempting to generalise the analysis to this case. From the brane-web perspective, a convenient starting point is the 5 -brane web with an $\mathrm{O}^{+}$plane for a $\operatorname{Sp}(k)$ theory with $N_{f}=N_{L}+N_{R}+1$ fundamental flavours. Without loss of generality, the additional flavour is chosen to be on the right-hand-side, such that

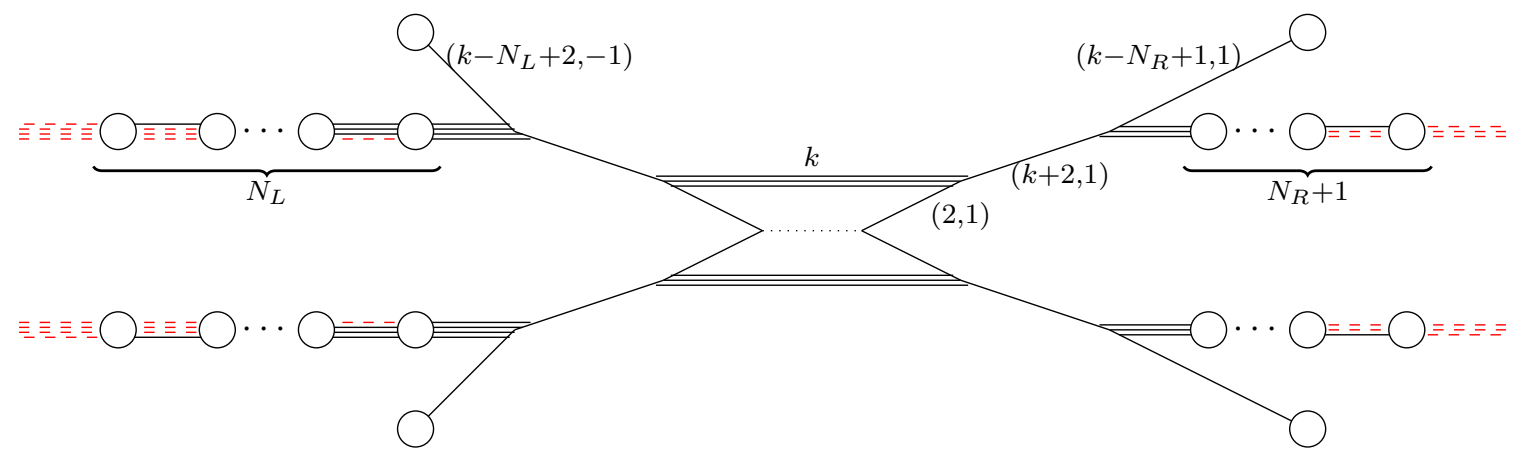

and then one can move one of the flavours on the right-hand-side onto the orientifold. The resulting physical 7-brane, from the merging of the half 7-brane and its mirror, can be 


\begin{tabular}{|c|cccccccccc|}
\hline Type IIB & $x^{0}$ & $x^{1}$ & $x^{2}$ & $x^{3}$ & $x^{4}$ & $x^{5}$ & $x^{6}$ & $x^{7}$ & $x^{8}$ & $x^{9}$ \\
\hline NS5 & $\times$ & $\times$ & $\times$ & $\times$ & $\times$ & $\times$ & & & & \\
D5 & $\times$ & $\times$ & $\times$ & $\times$ & $\times$ & & $\times$ & & & \\
$(p, q) 5$-brane & $\times$ & $\times$ & $\times$ & $\times$ & $\times$ & angle $\alpha$ & & & \\
O7 $^{-}$ & $\times$ & $\times$ & $\times$ & $\times$ & $\times$ & & & $\times$ & $\times$ & $\times$ \\
{$[p, q]$ 7-brane } & $\times$ & $\times$ & $\times$ & $\times$ & $\times$ & & & $\times$ & $\times$ & $\times$ \\
\hline
\end{tabular}

Table 8. Type IIB 5-brane web set-up with $\mathrm{O}^{-}$plane: $\times$indicates the space-time directions spanned by the various branes and the orientifold plane. A $(p, q) 5$-brane is a line of slope $\tan (\alpha)=$ $q \tau_{2} /\left(p+q \tau_{1}\right)$ in the $x^{5,6}$ plane where the axiodilaton is $\tau=\tau_{1}+i \tau_{2}$. In this paper all the brane webs are drawn for the value $\tau=i$, so that $\tan (\alpha)=q / p$.

split along the orientifold. Then, moving only one half 7-brane through the 5-branes to the left-hand-side results in

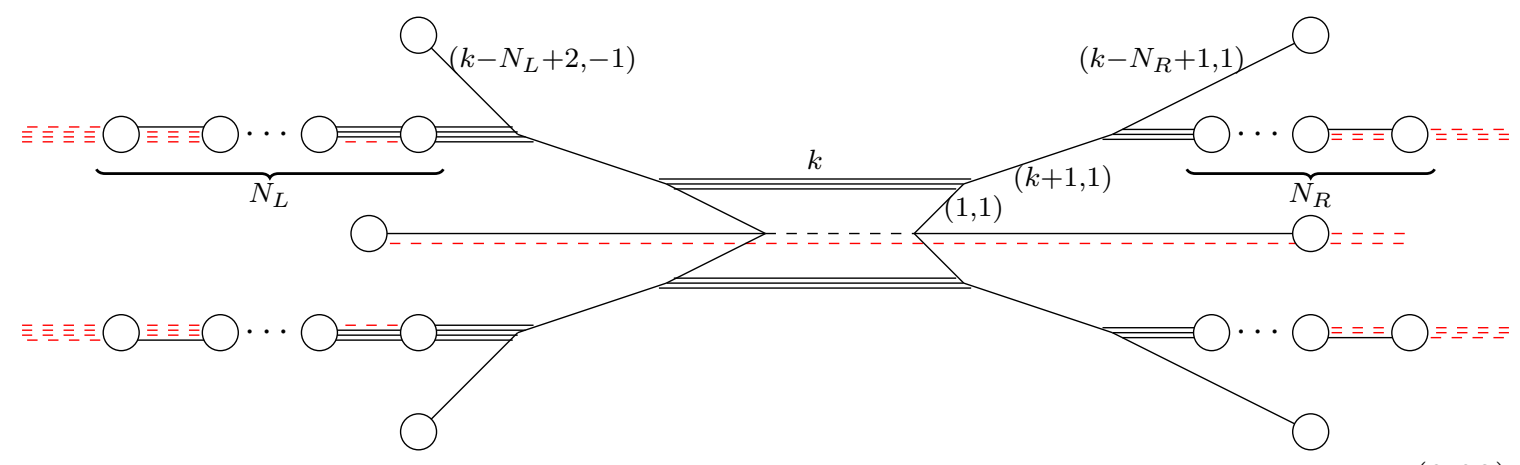

and one can send the two half 7 -branes which are on the orientifold towards $\pm \infty$. Via these steps, one has achieve a 5 -brane construction for a symplectic gauge group using an $\widetilde{\mathrm{O} 5}^{+}$ plane. Consequently, the brane web proposal for $\operatorname{Sp}(k)$ with $\tilde{N}_{f}=N_{L}+N_{R}=N_{f}-1$ from an $\mathrm{O}^{+}$plane is equivalent to that of $\mathrm{Sp}(k)$ with $N_{f}$ flavours from an $\widetilde{\mathrm{O}}^{+}$plane. Hence, there does not appear to be a new theory, which agrees with the findings of [10] and the $3 \mathrm{~d} \mathcal{N}=4$ analysis in [87].

\section{Comparison with $\mathrm{O}^{-}$construction}

While the main focus of this work are constructions of $5 \mathrm{~d} \mathcal{N}=1$ gauge theories and their UV fixed points using brane webs with O5 planes, it is instructive to compare the results to constructions with an $\mathrm{O} 7$ plane [9]. The space-time occupation of the various branes in this setup are summarised in table 8 . In this section, the brane webs with an $\mathrm{O}^{-}$ plane realising an $\operatorname{Sp}(k)$ gauge theory with $N_{f} \leq 2 k+5$ fundamental hypermultiplets are presented. The starting point is the massive Coulomb phase, which for $k$ being the rank 
of the gauge group and $N_{f}=N_{L}+N_{R}$ can be depicted as follows:

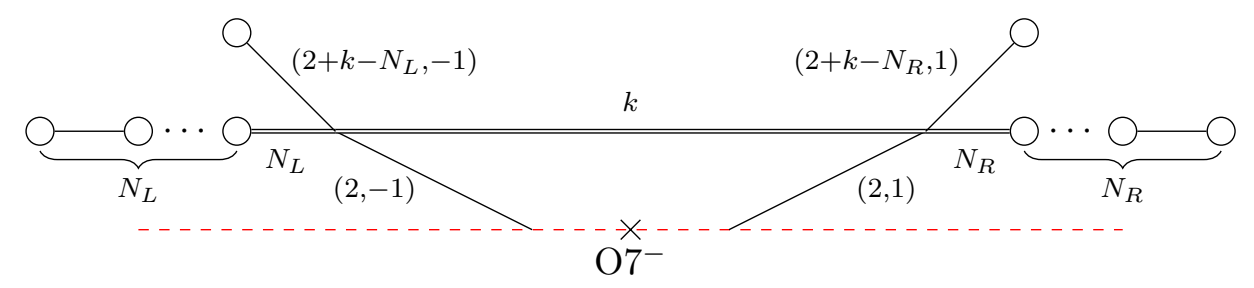

and masses of the hypermultiplets are chosen to be equal for convenience. For the purposes of this section, the decomposition of the number of flavours into $N_{f}=N_{L}+N_{R}$ is a convenient choice and has no impact on the results. As shown in [96], an $\mathrm{O}^{-}$orientifold is quantum mechanically resolved into a pair of mutually non-local $[p, q]$ 7-branes, such that the combined monodromy equals that of the $\mathrm{O}^{-}$. One common choice is given by a $[1,1] 7$-brane together with a $[1,-1] 7$-brane, which is denoted as blue and red respectively. Thus, then quantum mechanically corrected 5 -brane web becomes

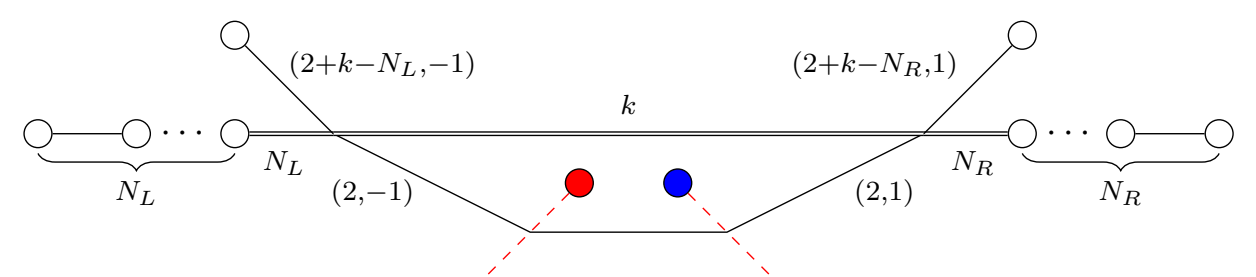

which can be brought into a more convenient form by moving the monodromy cuts. Accounting for the effects of the monodromy (A.3), one finds

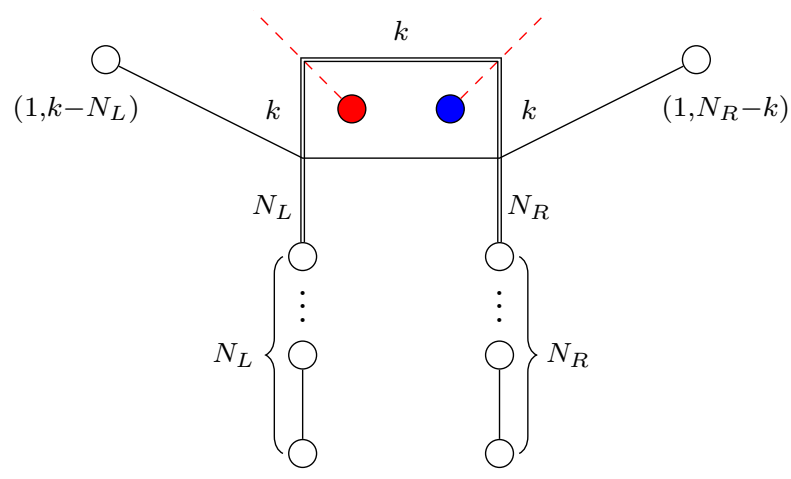

and the $[1, \pm 1] 7$-branes inside the web can be moved through the 5 -branes by accounting for brane creation, as in (A.4). Therefore, the 5-brane web with all 7-branes outside is given by

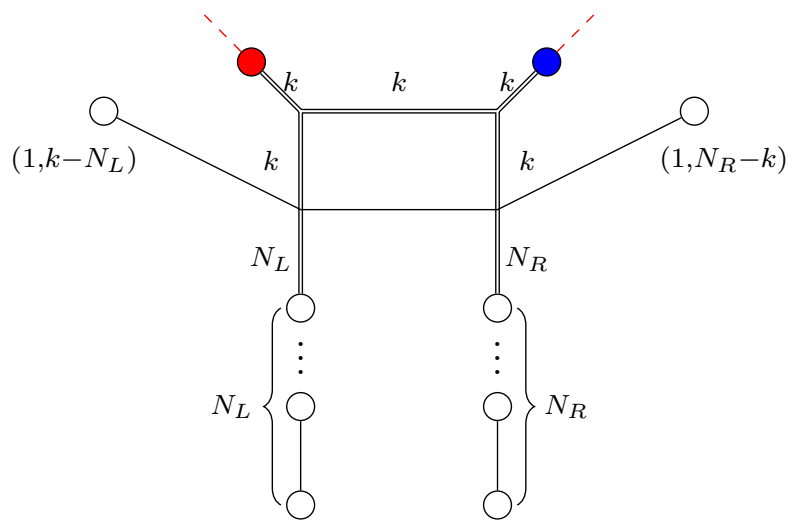


such that one can finally transition to the Higgs branch phase. For this, all masses are set to zero and one ends up with

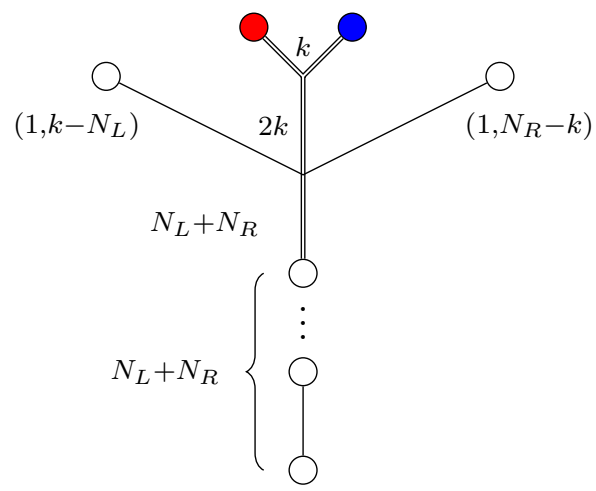

which is a 5-brane web ending on 7-branes without O5 planes. Therefore, the results of [24] apply. In other words, starting from (4.5), the associated magnetic quivers with only unitary magnetic gauge nodes are read off by [24, conjecture 1$]$.

\subsection{Finite coupling}

In the following, unitary magnetic quivers are computed for $\operatorname{Sp}(k)$ theories at finite coupling.

$\boldsymbol{N}_{\boldsymbol{f}} \leq \mathbf{2} \boldsymbol{k}+\mathbf{1}$. For small numbers of flavours the magnetic quiver depends on $N_{f}$ being even or odd. For even number of flavours one finds the following two maximal subdivisions:
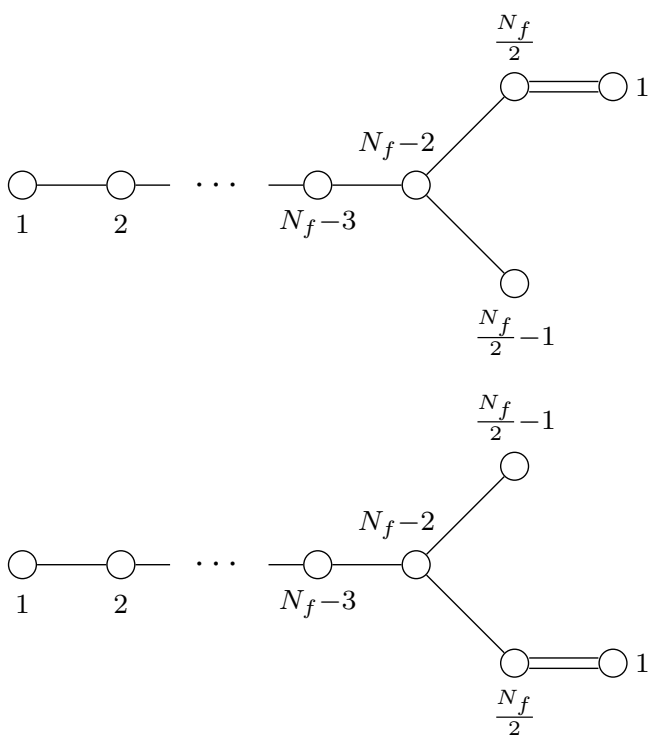
and the intersection of the cones associated to (4.6a) and (4.6b) in the Higgs branch is described by

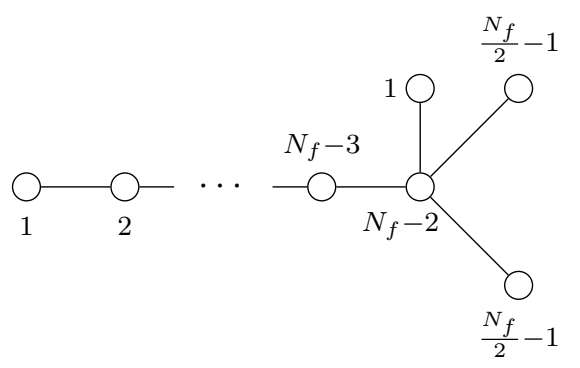

In contrast, for odd number of flavours there is only one maximal subdivision and the magnetic quiver becomes

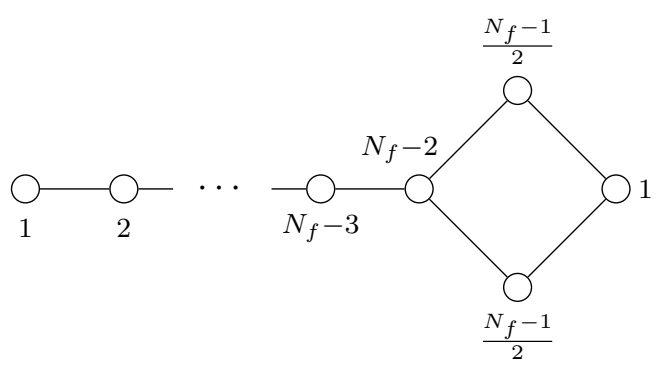

In both cases, one straightforwardly computes

$$
\operatorname{dim}_{\mathbb{H}} \mathcal{C}(4.6)=\operatorname{dim}_{\mathbb{H}} \mathcal{C}(4.7)=\frac{N_{f}\left(N_{f}-1\right)}{2} \quad \text { and } \quad G=\operatorname{SO}\left(2 N_{f}\right)
$$

due to the subset of balanced nodes, see A.3. In fact, the moduli spaces are known [94] to satisfy

$$
\begin{aligned}
\mathcal{C}(4.6 \mathrm{a}) \cup \mathcal{C}(4.6 \mathrm{~b}) & =\overline{\mathcal{O}}_{\mathrm{D}}^{\left[2^{N_{f}}\right]}, \quad \mathcal{C}(4.6 \mathrm{a}) \cap \mathcal{C}(4.6 \mathrm{~b})=\mathcal{C}(4.6 \mathrm{c})=\overline{\mathcal{O}}_{\mathrm{D}}^{\left[2^{N_{f}-2}, 1^{4}\right]} \\
\mathcal{C}(4.7) & =\overline{\mathcal{O}}_{\mathrm{D}}^{\left[2^{N_{f}-1}, 1^{2}\right]}
\end{aligned}
$$

As a remark, incomplete Higgsing has not been detailed in [94]; however, the magnetic quivers still have the same structure and thus the results apply. In particular, these results agree with the Higgs branch expectations (3.3)-(3.4) as well as the magnetic quivers results (3.9) and (3.15) derived from O5 planes.

$\boldsymbol{N}_{\boldsymbol{f}}>\mathbf{2} \boldsymbol{k}+\mathbf{1}$. For large number of flavours, the magnetic quiver shows uniform behaviour and is read off to be

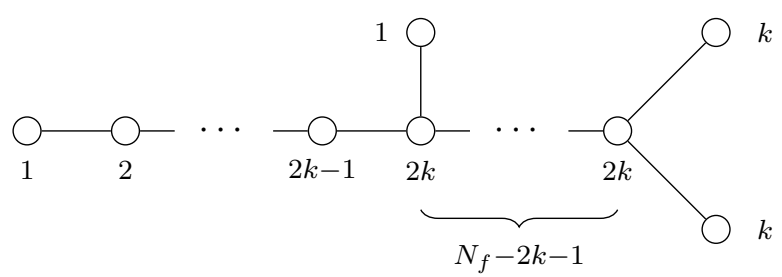


and one confirms that

$$
\operatorname{dim}_{\mathbb{H}} \mathcal{C}(4.10)=k\left(2 N_{f}-2 k-1\right) \quad \text { and } \quad G=\mathrm{SO}\left(2 N_{f}\right)
$$

which agrees with the Higgs branch expectation (3.1) as well as the magnetic quiver result (3.11) and (3.17) derived from O5 planes. In addition, one notes that (4.10) agrees with the $3 \mathrm{~d} \mathcal{N}=4$ mirror theory for $\operatorname{Sp}(k)$ gauge theory with $N_{f} \geq 2 k$ flavours derived in [97] [94, figure 4]. The moduli space

$$
\mathcal{C}(4.10)=\overline{\mathcal{O}}_{\mathrm{D}}^{\left[2^{2 k}, 1^{2 N_{f}-4 k}\right]}
$$

is known to be a nilpotent orbit closure.

\subsection{Infinite coupling}

In the following unitary magnetic quivers are computed for $\operatorname{Sp}(k)$ theories at infinite coupling.

$\boldsymbol{N}_{\boldsymbol{f}} \leq \mathbf{2 k}$. Suppose the number of flavours satisfies $N_{f} \leq 2 k$, then the infinite coupling regime is reached via global deformation of the brane web

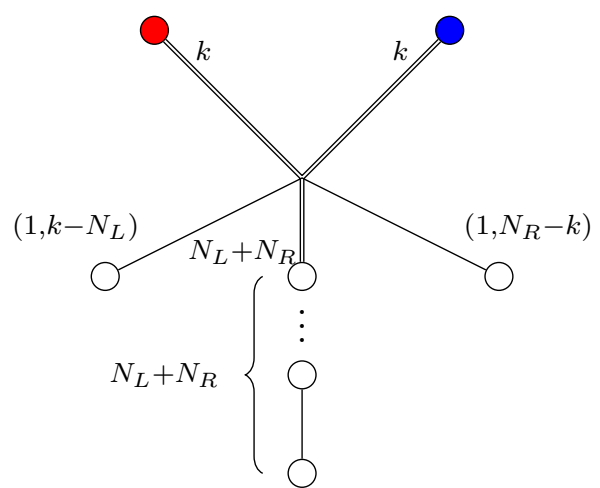

and magnetic quivers at infinite coupling can be read off by the usual rules. However, the result depends on $N_{f}$ being even or odd, similar to the finite coupling case. To begin with, suppose $N_{f}$ is even, then one finds two maximal subdivision with the following magnetic quivers:
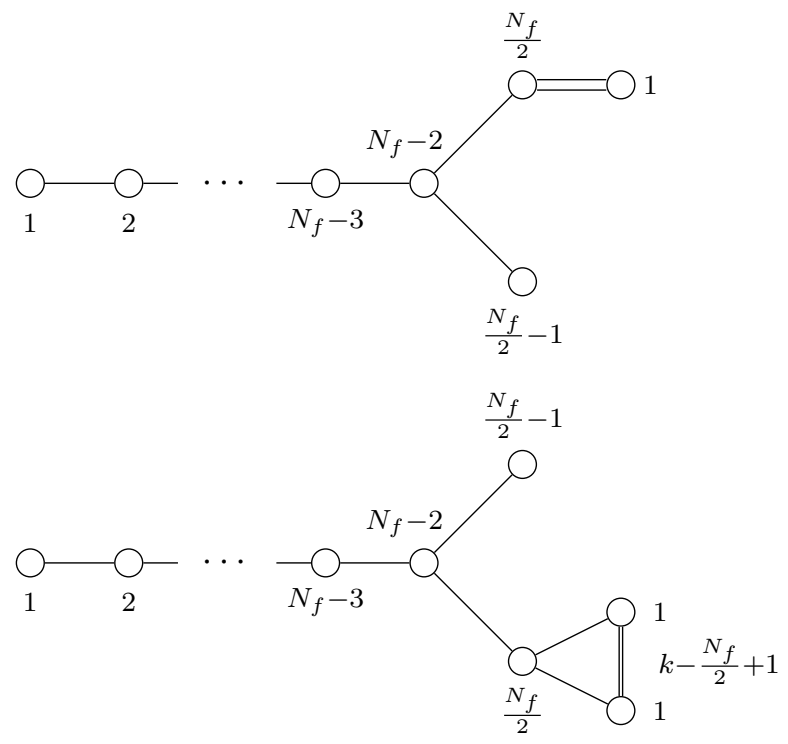
and the moduli space becomes the union of two cones. Note that (4.14a) coincides with the finite coupling results, while for (4.14b) one computes the following

$$
\operatorname{dim}_{\mathbb{H}} \mathcal{C}(4.14 \mathrm{~b})=\frac{1}{2} N_{f}\left(N_{f}-1\right)+1 \quad G=\mathrm{SO}\left(2 N_{f}\right) \times \mathrm{U}(1) .
$$

For odd number of flavours $N_{f}$, there exists only one maximal subdivision and the magnetic quivers is read off to be

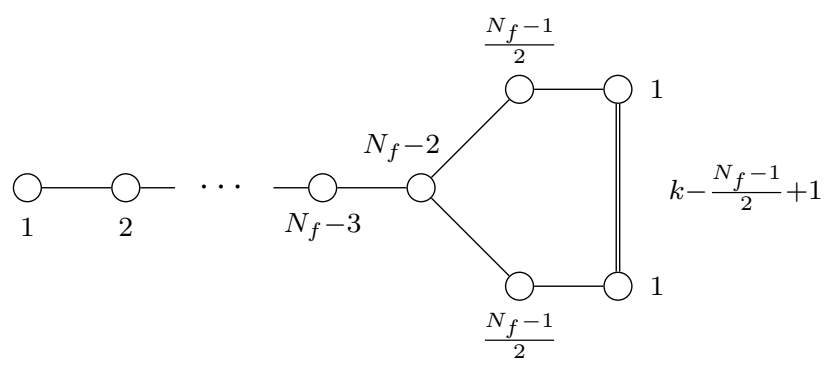

and, as above, one concludes that

$$
\operatorname{dim}_{\mathbb{H}} \mathcal{C}(4.16)=\frac{1}{2} N_{f}\left(N_{f}-1\right)+1 \quad G_{J}=\mathrm{SO}\left(2 N_{f}\right) \times \mathrm{U}(1) .
$$

Both cases (4.14)-(4.16) correctly reproduce the number of additional Higgs branch directions at infinite coupling [9]. Comparing to the results (3.19) and (3.25) from the O5 plane construction, the $\mathrm{O}^{-}$results do indicate the existence of multiple cones, which are not currently visible in the $\mathrm{O} 5$ analysis.

$\boldsymbol{N}_{\boldsymbol{f}}=\mathbf{2} \boldsymbol{k}+\mathbf{1}$. Increasing the number of flavours to $N_{f}=2 k+1$, the 5 -brane web has to be evaluated more carefully. In detail, it becomes

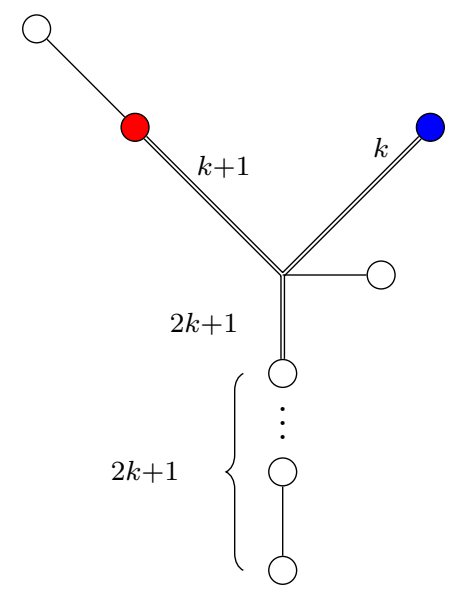

and the associated magnetic quiver is

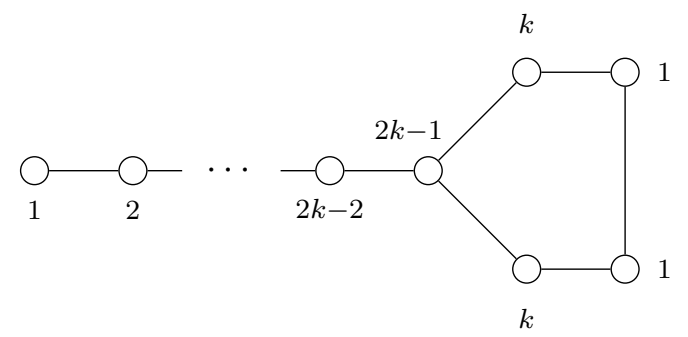


The Coulomb branch dimension is readily computed

$$
\operatorname{dim}_{\mathbb{H}} \mathcal{C}(4.19)=\frac{1}{2} N_{f}\left(N_{f}-1\right)+1 \quad \text { and } \quad G=\mathrm{SO}\left(2 N_{f}\right) \times \mathrm{U}(1)
$$

The addition in Higgs branch dimension agrees with the expectation [9] as well as the dimension of the magnetic quiver (3.25) from the O5 plane construction. Furthermore, (4.19) reduces for $k=1$ to the affine $a_{4}$ quiver whose Coulomb branch equals $\overline{\mathcal{O}}_{A_{4}}^{\min }$.

$\boldsymbol{N}_{\boldsymbol{f}}=\mathbf{2} \boldsymbol{k}+\mathbf{2}$. Next, for $N_{f}=2 k+2$ fundamental flavours the 5 -brane web is given by

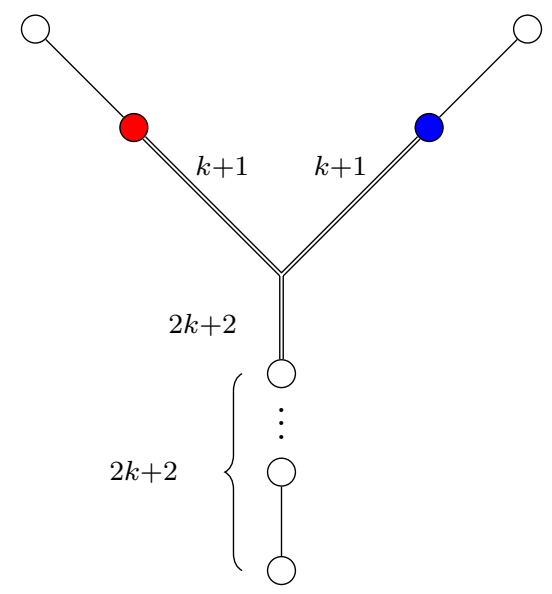

and the corresponding magnetic quiver is determined to be

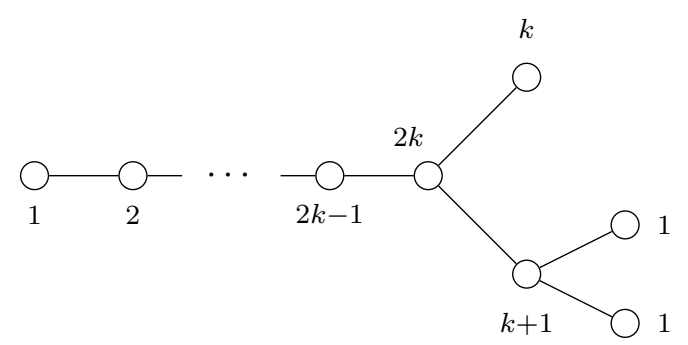

The associated Coulomb branch is of dimension

$$
\operatorname{dim}_{\mathbb{H}} \mathcal{C}(4.22)=\frac{1}{2} N_{f}\left(N_{f}-1\right)+1 \quad \text { and } \quad G=\mathrm{SO}\left(2 N_{f}\right) \times \mathrm{U}(1) .
$$

Note that the case $k=1$ reduces to the affine $d_{5}$ quiver, i.e. the quiver whose Coulomb branch equals $\overline{\mathcal{O}}_{\mathrm{D}_{5}}^{\min }$. 
$N_{f}=2 \boldsymbol{k}+\mathbf{3}$. Increasing the number of flavours further $N_{f}=2 k+3$, the brane web is given by

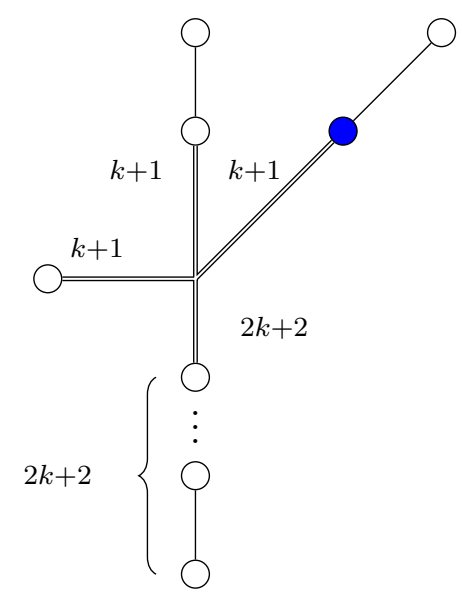

such that the magnetic quiver reads

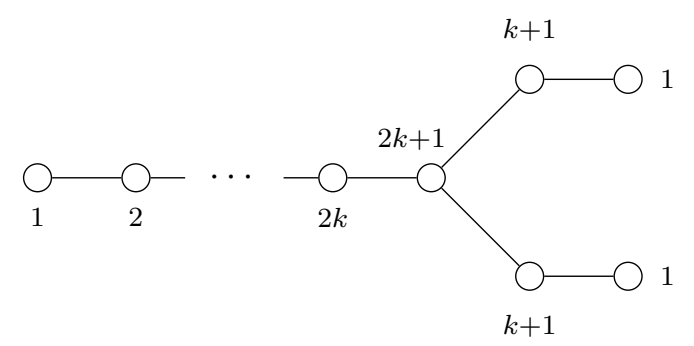

One readily confirms that

$$
\operatorname{dim}_{\mathbb{H}} \mathcal{C}(4.25)=\frac{1}{2} N_{f}\left(N_{f}-1\right)+1 \quad \text { and } \quad G=\mathrm{SO}\left(2 N_{f}\right) \times \mathrm{U}(1),
$$

which indicates one additional Higgs branch direction at infinite coupling, in agreement with [9]. Moreover, note that the $k=1$ case reduces (4.25) to the affine $e_{6}$ quiver, i.e. the quiver with Coulomb branch $\overline{\mathcal{O}}_{E_{6}}^{\min }$.

$\boldsymbol{N}_{\boldsymbol{f}}=\mathbf{2} \boldsymbol{k}+\mathbf{4}$. The brane web for the field theory limit $N_{f}=2 k+4$ is given by

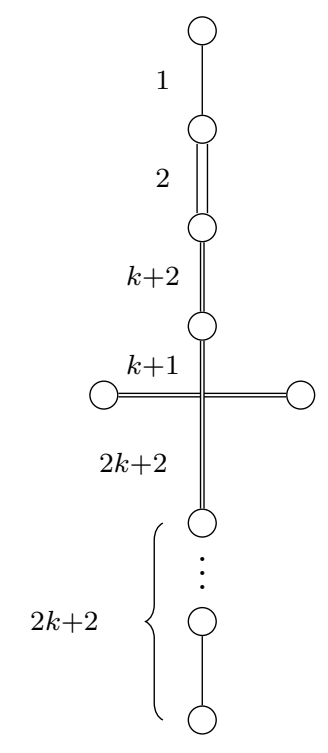


and the associated magnetic quiver is

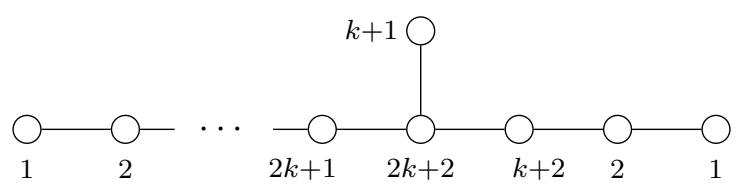

One readily computes

$$
\operatorname{dim}_{\mathbb{H}} \mathcal{C}(4.28)=\frac{1}{2} N_{f}\left(N_{f}-1\right)+2 \quad \text { and } \quad G=\mathrm{SO}\left(2 N_{f}\right) \times \mathrm{SU}(2)
$$

which indicates two additional Higgs branch directions in comparison to the finite coupling case. This agrees with the expectation of [9] as well as the magnetic quiver (3.22) from O5 plane construction. In particular, note that for $k=1$ the quiver (4.28) reduces to the affine $e_{7}$ quiver, meaning that its Coulomb branch is $\overline{\mathcal{O}}_{E_{7}}^{\text {min }}$.

$\boldsymbol{N}_{\boldsymbol{f}}=\mathbf{2 k}+\mathbf{5}$. Lastly, considering the maximal number of flavours, the brane web is given by

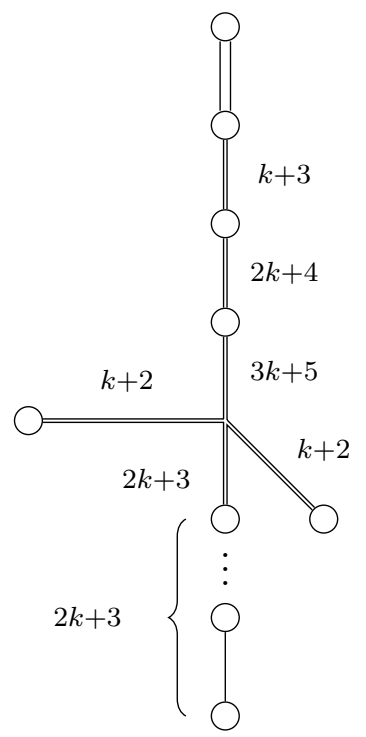

and one derives the magnetic quiver to be

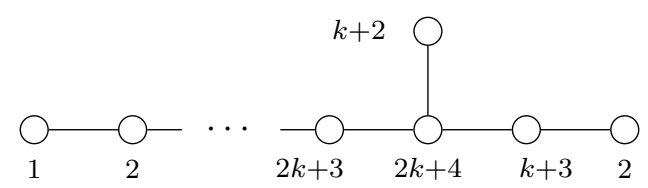

Computing the moduli space properties reveals

$$
\operatorname{dim}_{\mathbb{H}} \mathcal{C}(4.31)=\frac{1}{2} N_{f}\left(N_{f}+1\right)+1 \quad \text { and } \quad G=\mathrm{SO}\left(2 N_{f}+2\right),
$$

which matches the dimension of (3.29) from the O5 construction. Moreover, for $k=1$ one observes that (4.31) reduces to the affine $e_{8}$ quiver such that its Coulomb branch equals $\overline{\mathcal{O}}_{E_{8}}^{\min }$. 


\subsection{Duality with 5d SQCD}

The unitary magnetic quiver descriptions for the $5 \mathrm{~d} \operatorname{Sp}(k)$ theories derived above admit an interesting property. Recalling the infinite coupling Higgs branch descriptions for 5d SQCD in [24, section 5.4] one finds that the following theories have the same infinite coupling Higgs branch descriptions in terms of magnetic quivers

- $E_{8}$ family (4.31): $5 \mathrm{~d} \mathrm{Sp}(k)$ with $N_{f}=2 k+5$ flavours $\leftrightarrow 5 \mathrm{~d} \mathrm{SU}(k+1)$ with $N_{f}$ flavours and CS-level $\kappa= \pm \frac{1}{2},[24$, table 20]

- $E_{7}$ family (4.28): $5 \mathrm{~d} \mathrm{Sp}(k)$ with $N_{f}=2 k+4$ flavours $\leftrightarrow 5 \mathrm{~d} \mathrm{SU}(k+1)$ with $N_{f}$ flavours and CS-level $\kappa= \pm 1$, [24, table 19]

- $E_{6}$ family (4.25): $5 \mathrm{~d} \mathrm{Sp}(k)$ with $N_{f}=2 k+3$ flavours $\leftrightarrow 5 \mathrm{~d} \mathrm{SU}(k+1)$ with $N_{f}$ flavours and CS-level $\kappa= \pm \frac{3}{2}$, [24, table 18]

- $E_{5}$ family (4.22): $5 \mathrm{~d} \mathrm{Sp}(k)$ with $N_{f}=2 k+2$ flavours $\leftrightarrow 5 \mathrm{~d} \mathrm{SU}(k+1)$ with $N_{f}$ flavours and CS-level $\kappa= \pm 2$, [24, table 17]

- $E_{4}$ family (4.19): $5 \mathrm{~d} \mathrm{Sp}(k)$ with $N_{f}=2 k+1$ flavours $\leftrightarrow 5 \mathrm{~d} \mathrm{SU}(k+1)$ with $N_{f}$ flavours and CS-level $\kappa= \pm \frac{5}{2}$, [24, table $\left.16(\mathrm{~V})\right]$

- Case (4.14): $5 \mathrm{~d} \mathrm{Sp}(k)$ with even number of flavours $N_{f} \leq 2 k \leftrightarrow 5 \mathrm{~d} \mathrm{SU}(k+1)$ with $N_{f}$ flavours and CS-level $\kappa= \pm\left(k+3-\frac{N_{f}}{2}\right)$, [24, table $\left.16(\mathrm{IV}) \&(\mathrm{~V})\right]$

- Case (4.16): $5 \mathrm{~d} \mathrm{Sp}(k)$ with odd number of flavours $N_{f} \leq 2 k \leftrightarrow 5 \mathrm{~d} \mathrm{SU}(k+1)$ with $N_{f}$ flavours and CS-level $\kappa= \pm\left(k+3-\frac{N_{f}}{2}\right),[24$, table $16(\mathrm{~V})]$

As summarised in table 1 , the magnetic quivers hint on the $5 \mathrm{~d} \mathcal{N}=1$ duality

$$
\mathrm{Sp}(k), N_{f} \text { flavours } \longleftrightarrow \mathrm{SU}(k+1)_{ \pm\left(k+3-\frac{N_{f}}{2}\right)}, N_{f} \text { flavours }
$$

In fact, the duality (4.33) has been conjectured in [16] and observed from 5-brane webs in [18] or from geometric studies in [53, eq. (4.2)] and [55, eq. (2.225)].

\subsection{Two realisations}

Another immediate observations follows from the construction of $5 \mathrm{~d} \operatorname{Sp}(k)$ theories via $\mathrm{O} 5$ planes or $\mathrm{O} 7$ planes. In other words, for each $5 \mathrm{~d} \operatorname{Sp}(k)$ theory with $N_{f}$ flavours, there exists

(i) a unitary-orthosymplectic (or orthosymplectic) magnetic quiver from the $\mathrm{O}^{-}$plane construction of section 3 , and

(ii) a unitary magnetic quiver from the $\mathrm{O}^{-}$construction of section 4 .

Table 1 contrasts the quivers in each case. For consistency, one needs to verify that the moduli spaces associated to both types of magnetic quiver are the same, or at least isomorphic. In a companion paper [80], the Hilbert series analysis is presented and the results confirm the suspected agreement.

\subsection{Problem of two cones}

From the construction of unitary magnetic quivers, it is clear that the (classical as well as infinite coupling) Higgs branch of $\operatorname{Sp}(k)$ with an even number of flavours $N_{f} \leq 2 k$, is a union of two cones. However, in the O5 construction of section 3 only one cone is visible. Note that a similar problem exists for constructions of 3 dimensional theories in Type IIB 
systems of NS5, D3, D5 with an O3 plane versus an O5 plane construction. One crucial difference between the unitary and orthosymplectic quivers for D-type global symmetry is the visibility of the spinor nodes in the unitary construction. To be specific, focus on the finite coupling case. The classical Higgs branch is the closure of a very even height two $\mathrm{O}\left(2 N_{f}\right)$ nilpotent orbit closure. The $\mathrm{O}\left(2 N_{f}\right)$ very even orbit is known to be a union of two $\mathrm{SO}\left(2 N_{f}\right)$ orbits [95], and the Higgs branch is a union of two cones. For a unitary quiver, one is able to make a clear distinction between the two cones and also detail the intersection of the cones. The quivers representing the two cones are related by a non-trivial $\mathbb{Z}_{2}$-action, and the individual cones are isomorphic. The non-trivial intersection is given by a quiver invariant under the $\mathbb{Z}_{2}$-action. The relevant quivers are as follows:

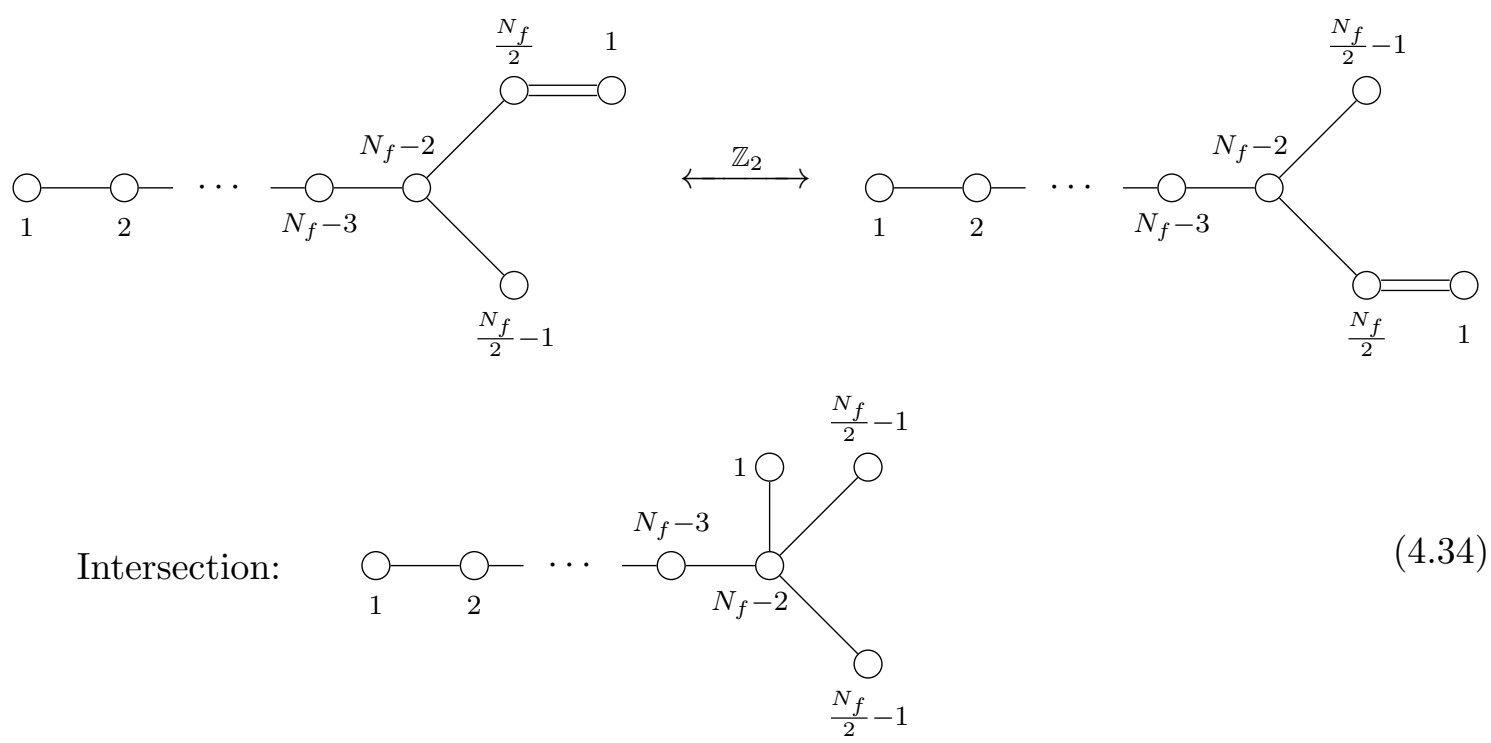

In contrast, the construction of the two cones as Coulomb branches of orthosymplectic quivers is slightly different. Here, the two cones cannot be made distinct, the relevant quivers are as follows:
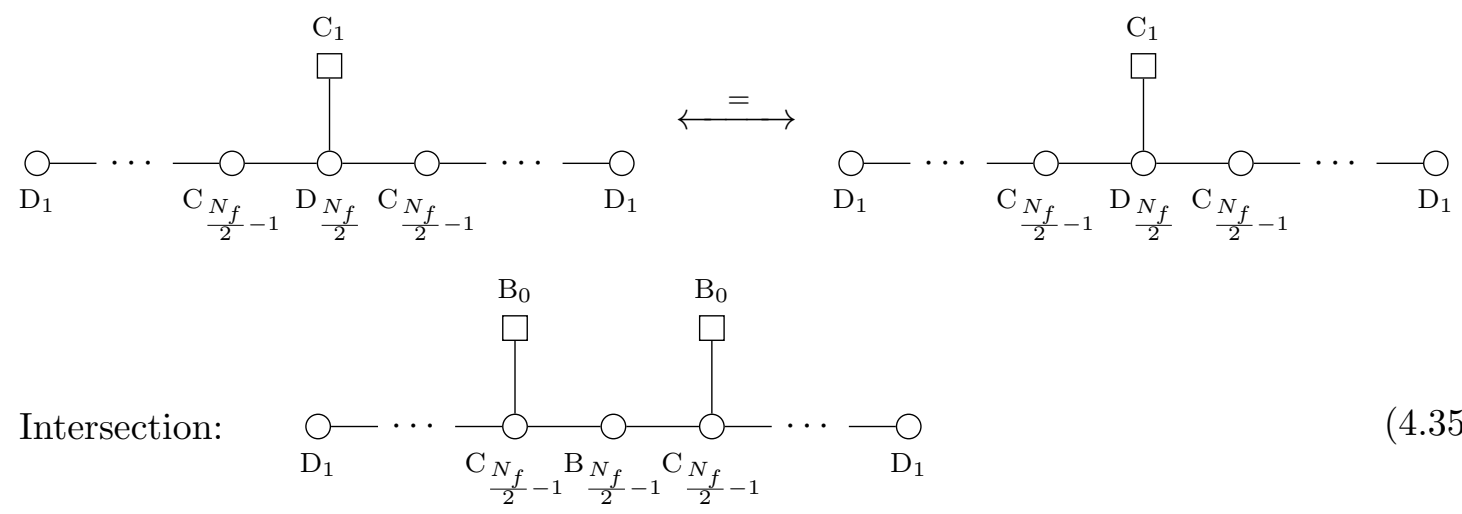

So the inability to distinguish two cones may come from the inability to distinguish the magnetic quivers in the first place. This changes at infinite coupling, where only one of the cones grows with respect to the finite coupling case, and one can in principle make a 
distinction between the quivers representing the two cones. Still there is no way to identify which of the cones was enhanced and only the magnetic quiver representing the enhanced cone can be identified.

\section{Hasse diagrams and quiver subtraction}

This section is dedicated to the study of Hasse diagrams of the theories discussed in this note. The finite coupling Hasse diagrams are known [69], so only the infinite coupling Hasse diagrams are computed in this section. The results for both finite and infinite coupling are juxtaposed in tables 2 and 3 .

Following the spirit of [70] one can derive the Higgs branch Hasse diagrams of the theories considered by using the brane web construction of section 3. In principle, one could also use the algorithm of quiver subtraction, as introduced in [73, 74] and further developed for unitary quivers in [70] and for framed (flavoured) orthosymplectic quivers in [81]. However, the orthosymplectic quivers appearing in table 1 are not framed, and may involve unitary gauge groups, and as a consequence the currently known algorithm needs to be extended. After one transition, framed quivers appear and the quiver subtraction algorithm of $[73,74,81]$ yields the rest of the Hasse diagram.

The results of section 2.3 and 3 show that unitary-orthosymplectic magnetic quivers naturally arise and create the need for an adaptation of quiver subtraction to this class of theories. In this section, the quiver subtraction algorithm is derived from brane set-ups, which then allows one to derive the Hasse diagram.

\section{$5.1 \quad E_{8}$ family}

Consider the brane web (3.28), which describes the Higgs branch of $\operatorname{Sp}(k)$ with $N_{f}=2 k+5$ flavours at infinite coupling. Opening up a Coulomb branch modulus one has to be careful to take the S-rule as well as charge conservation into account. In order to respect the S-rule from the point of view of the $[0,1] 7$-brane, the two $(0,1) 5$-branes have to split into a $(2,1)$ and $(2,-1) 5$-brane. Moreover, charge conservation on the orientifold implies that there have to be 4 full $(1,0) 5$-branes on the left-hand-side and right-hand-side. The S-rule further determines that every half $(1,0) 5$-brane has to end on its own half $[1,0] 7$-brane. The central part of the resulting web is

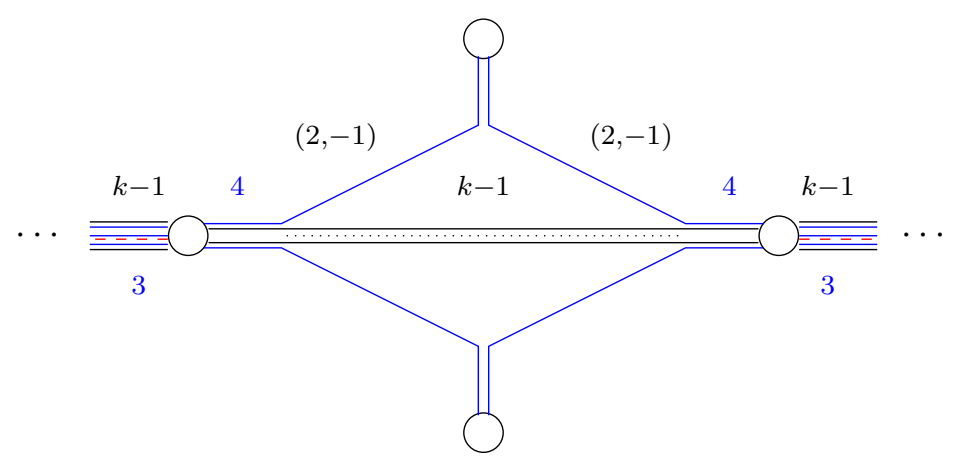

such that the blue 5-branes subweb can no longer split and is stuck to the orientifold. Thus, this subweb contributes as a flavour to both, the magnetic gauge group on its left 
and right. In more detail, the magnetic quiver corresponding to (5.1) is read off to be

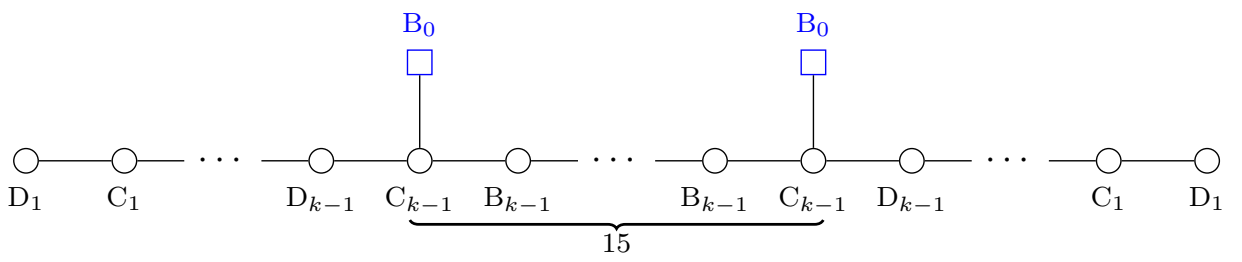

The blue piece can now be considered on its own and can be recognised as the $E_{8}$ brane system (2.8), with magnetic quiver given in (2.9). The procedure on the brane web above corresponds to a quiver subtraction of unframed quivers resulting in a quiver with framing, which was discussed for this $e_{8}$ transition in [65].

Having reduced the problem to a familiar setting, one can now employ the rules for quiver subtraction of framed linear orthosymplectic quivers [73, 81]. As a result, one obtains the following Hasse diagram:

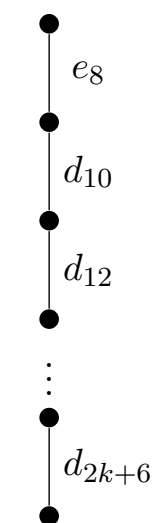

which matches the Hasse diagram [70, table 30] obtained from the unitary magnetic quiver (4.31). For $k=1$, the Hasse diagram reduces to a single $e_{8}$ transition.

\section{$5.2 \quad E_{7}$ family}

Next, consider the brane web (3.21) for the Higgs branch of $\operatorname{Sp}(k)$ with $N_{f}=2 k+4$ flavours at infinite coupling. In this case there are two ways to open up a single Coulomb branch modulus. On the one hand, one can repeat the same transition as for the $E_{8}$ family, given that $k>1$ :

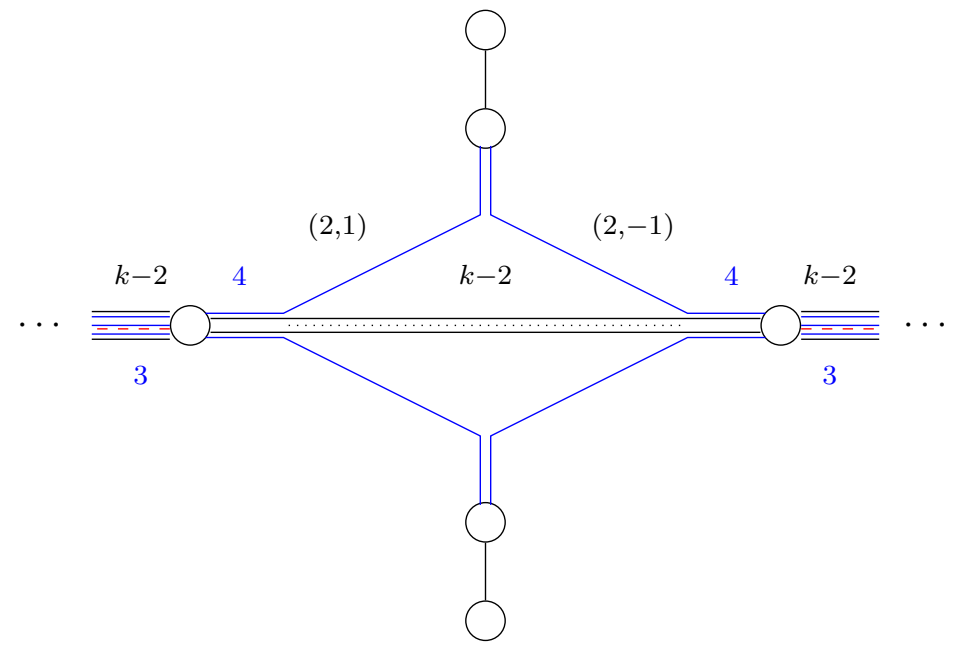


Consequently, the Hasse diagram has a $e_{8}$ transition at the top. The structure of the brane web (5.4) implies that the Higgs branch is described by a magnetic quiver which is a disjoint union of two quivers

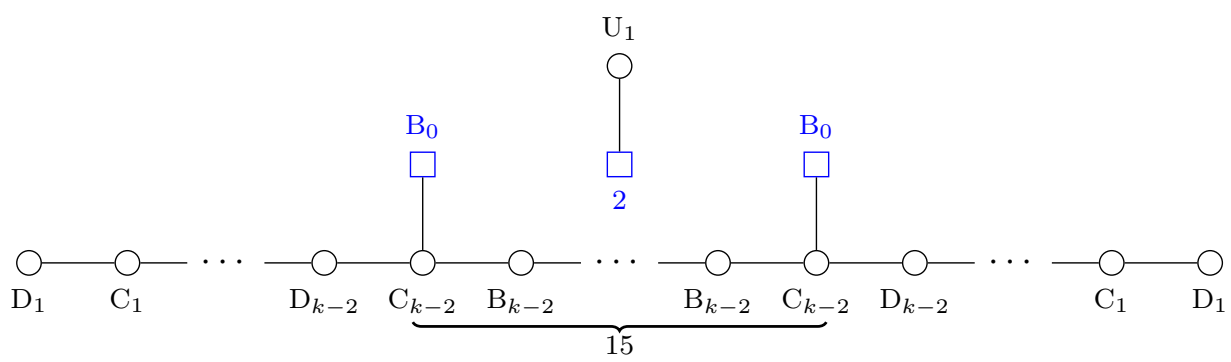

which is a framed orthosymplectic quiver together with a framed unitary quiver.

On the other hand, for $k \geq 1$ one can open up a different Coulomb branch modulus in the brane web (3.21) via

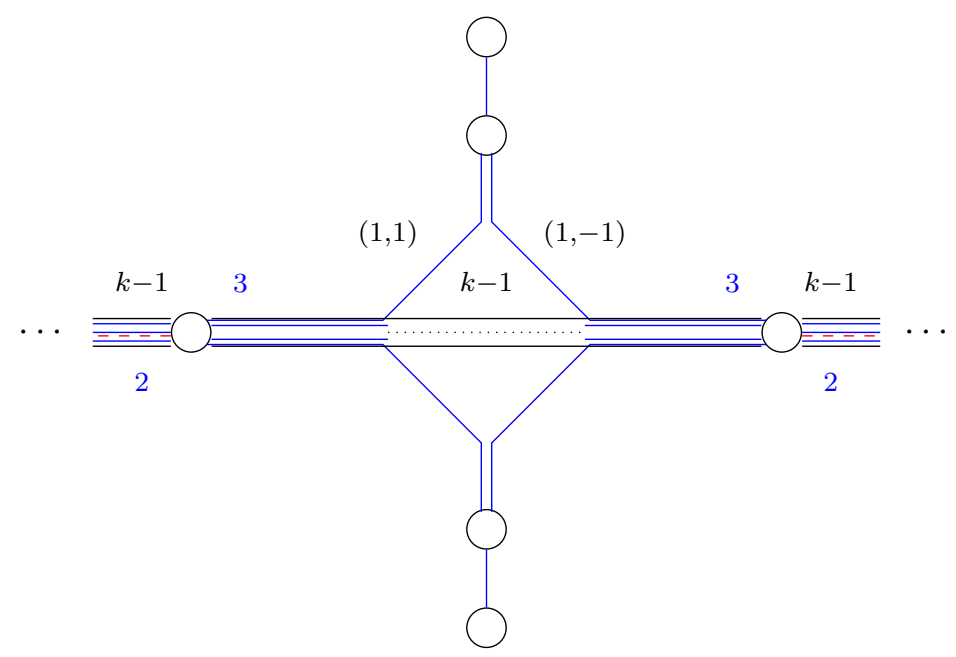

such that the blue brane subweb corresponds to the $E_{7}$ brane configuration (2.16) whose magnetic quiver is (2.17). For the Higgs branch described by the brane web (5.6) one reads off the following magnetic quiver:

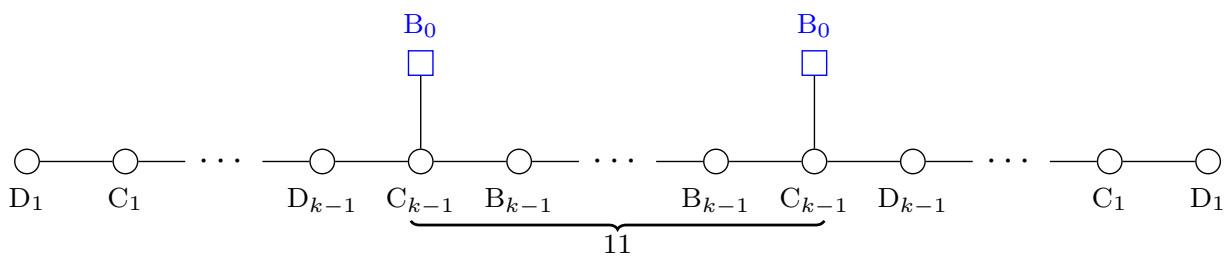

which is again a framed orthosymplectic quiver.

As a consequence, both ways to open up the minimal amount of Coulomb branch directions result in Higgs branches that are describable by framed orthosymplectic (or unitary) magnetic quivers (5.5) and (5.7). Therefore, the known algorithms for quiver 
subtraction then allow to derive the full Hasse diagram

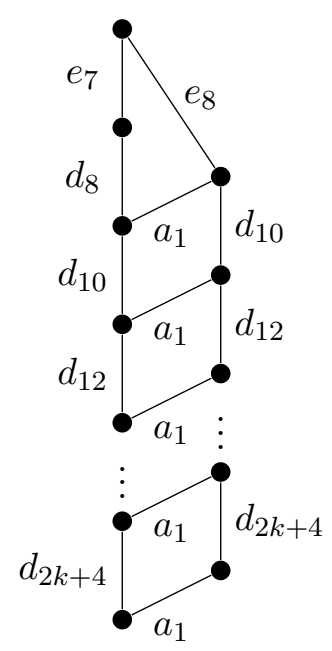

which agrees with the Hasse diagram [70, table 29] computed from the unitary magnetic quiver (4.28). For $k=1$, the Hasse diagram reduces to a single $e_{7}$ transition.

\section{$5.3 \quad E_{6}$ family}

Moving on to the brane web (3.24) for the Higgs branch of $\operatorname{Sp}(k)$ with $N_{f}=2 k+3$ flavours at infinite coupling, one recognises that there is exactly one option to open up a minimal Coulomb branch direction. In the brane web, the central part becomes

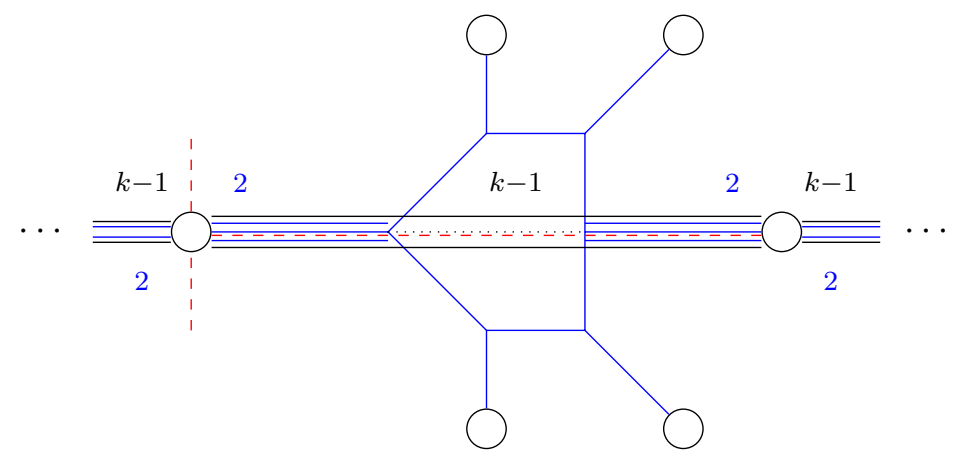

and the blue brane subweb is identified as the $E_{6}$ configuration (2.24), whose magnetic quiver is (2.25). For the remaining Higgs branch degrees of freedom in (5.9) one derives the following magnetic quiver

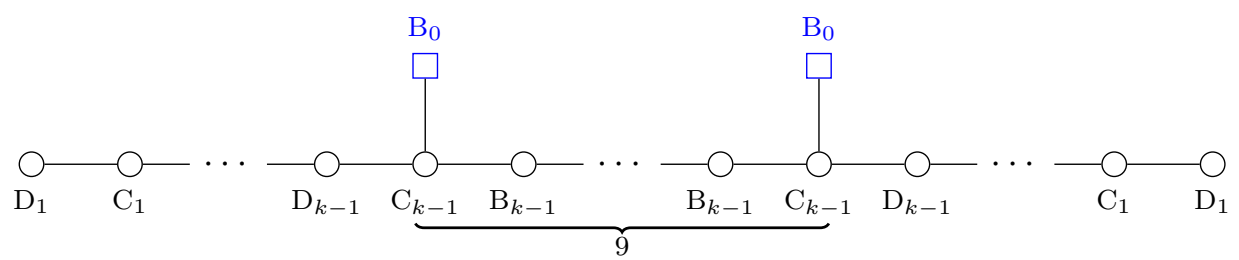

which is a framed orthosymplectic quiver. 
Again, as the Higgs branch after the $e_{6}$ transition is described by a framed quiver (5.10), the quiver subtraction algorithm allows one to complete the Higgs branch Hasse diagram. In detail, one obtains

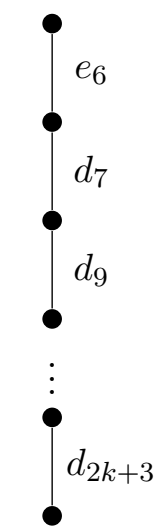

and one can verify the agreement with the Hasse diagram [70, table 28] obtained from the unitary magnetic quiver (4.25). For $k=1$, the Hasse diagram reduces to a single $e_{6}$ transition.

\section{$5.4 \quad E_{5}$ family}

Consider the brane web (3.18) for the Higgs branch of $\operatorname{Sp}(k)$ with $N_{f}=2 k+2$ flavours at infinite coupling. In order to open up the minimal number of Coulomb branch moduli, one finds that there is only one possibility

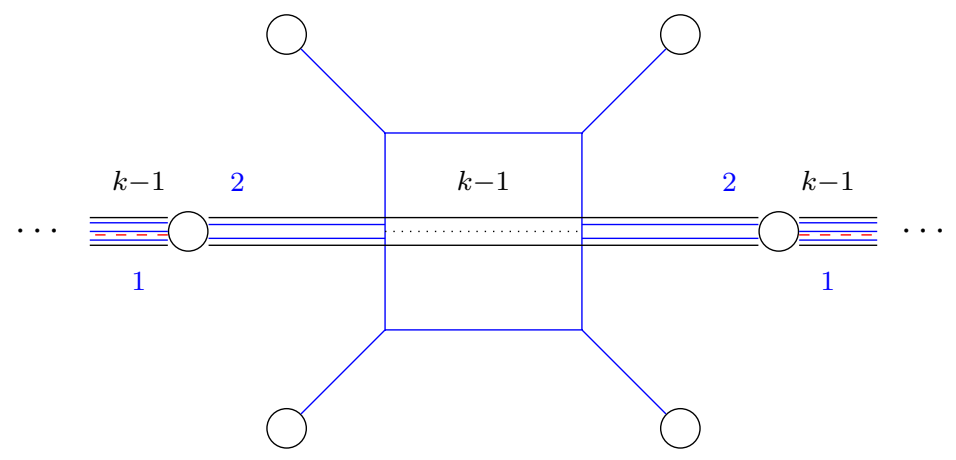

where the blue brane subweb corresponds to the $E_{5}$ configuration (2.32), with magnetic quiver (2.33). For the remaining Higgs branch directions in (5.12) one finds the magnetic quiver

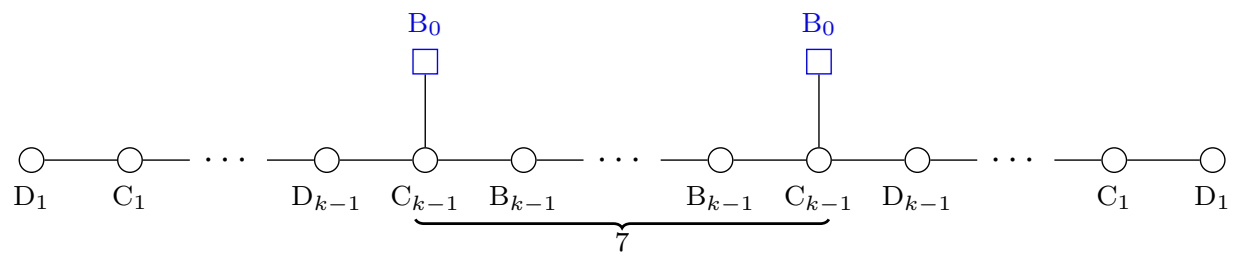

which is again a framed orthosymplectic quiver. 
Hence, the full Higgs branch Hasse diagram can then be derived from the framed starting point (5.13) via quiver subtraction. The Hasse diagram reads

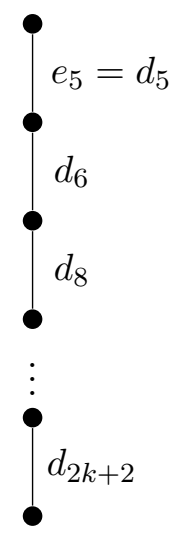

which coincides with the Hasse diagram [70, table 27] derived from the unitary magnetic quiver (4.22). For $k=1$, the Hasse diagram reduces to a single $e_{5}=d_{5}$ transition.

\section{$5.5 \quad E_{4}$ family}

Next, moving on to the brane web (3.24) for the Higgs branch of $\operatorname{Sp}(k)$ with $N_{f}=2 k+1$ flavours at infinite coupling. The minimal choice of opening up Coulomb branch directions is

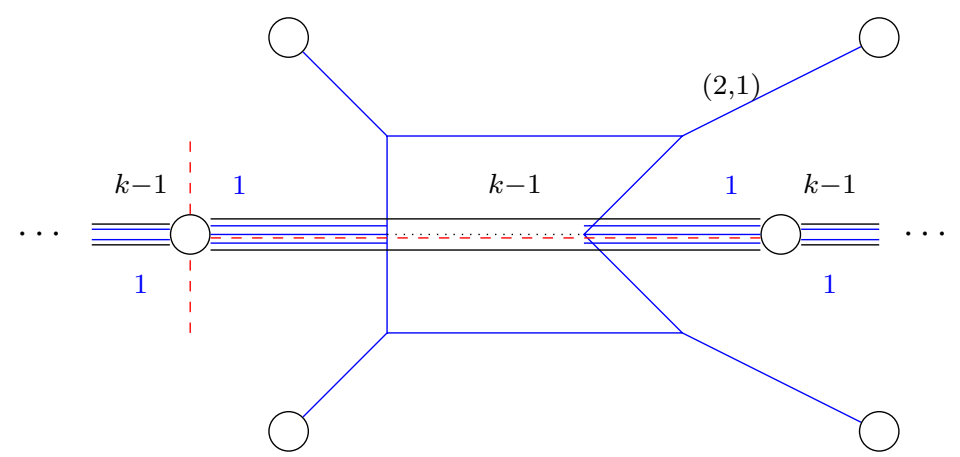

where the blue brane subweb corresponds to the $E_{4}$ configuration (2.40), with magnetic quiver (2.41). The magnetic quiver for (5.15) reads

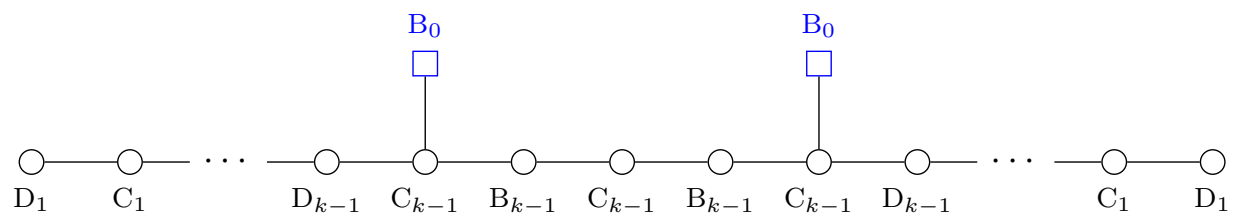


The remaining Hasse diagram can be derived by the same techniques as discussed above and one finds

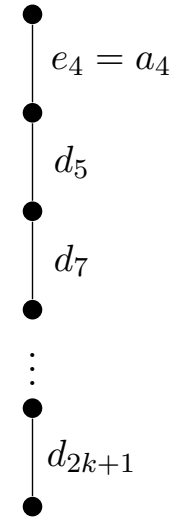

which agrees with the Hasse diagram [70, table 26] obtained from the unitary magnetic quiver (4.19). For $k=1$, the Hasse diagram reduces to a single $e_{4}=a_{4}$ transition.

\section{$5.6 \quad E_{3}$ family}

In the case of the generalised $E_{3}$ family, the brane web (3.18) for the Higgs branch of $\operatorname{Sp}(k)$ with $N_{f}=2 k$ flavours at infinite coupling does only give rise to one cone from the expected union of two cones. It is then not surprising that the web only allows one to identify the $a_{2}$ part of $e_{3}=a_{2} \cup a_{1}$ transition. In more detail, opening up the minimal number of Coulomb branch directions looks as follows:

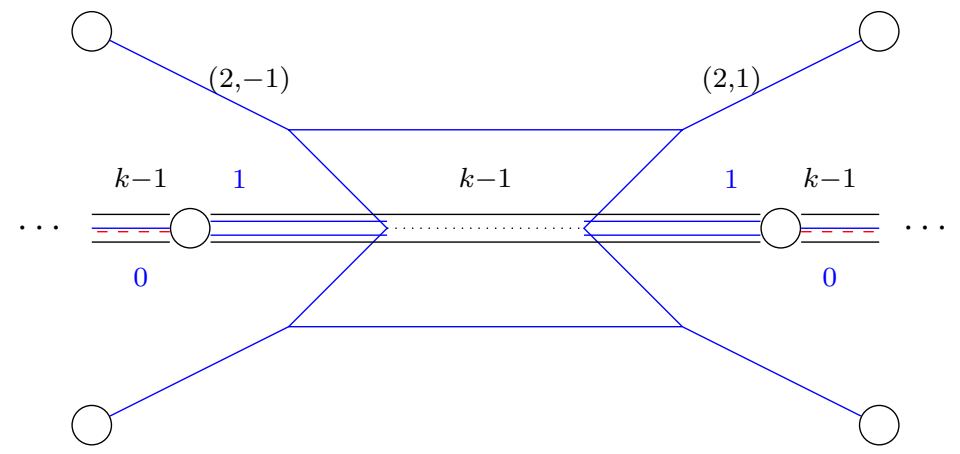

where the blue brane subweb corresponds to the $E_{3}$ configuration (2.47), with magnetic quiver (2.48). The magnetic quiver for (5.18) reads

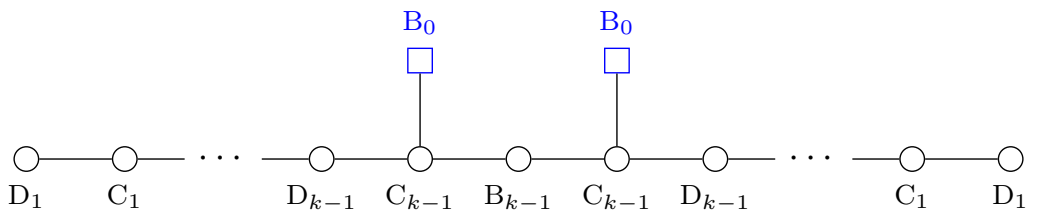


Taking (5.18) as a starting point, using quiver subtraction of framed orthosymplectic quivers, one obtains the black part of the following Hasse diagram:

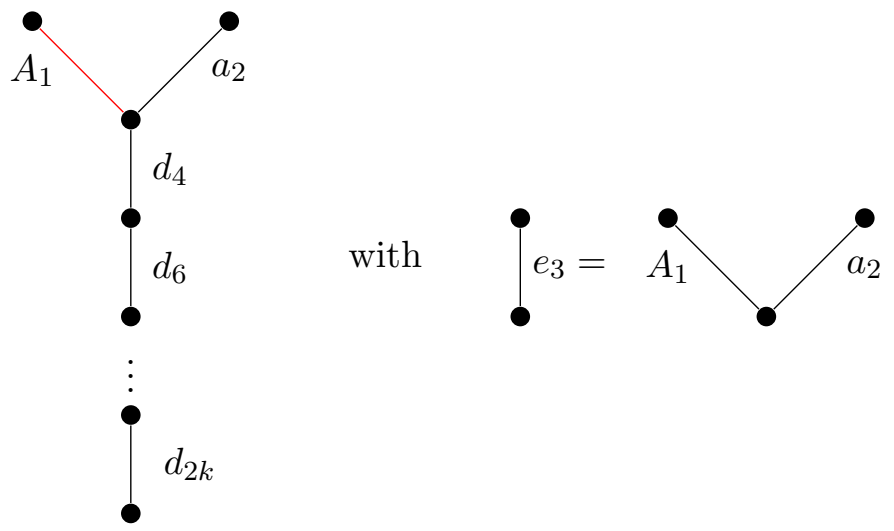

The $A_{1}$ transition indicated in red seems beyond the reach of the brane web (3.18) and it is currently not possible to identify it in this construction. Comparing with the Hasse diagram [70, table 6] obtained from the unitary magnetic quivers, one finds the black part of (5.20) from (4.14b) and the red transition from (4.14a). For $k=1$, the Hasse diagram reduces to a single $e_{3}$ transition.

\section{$5.7 \quad E_{2}$ family}

For the Higgs branch of $\operatorname{Sp}(k)$ with $N_{f}=2 k-1$ flavours at infinite coupling, the brane web is given in (3.24). The expectation is to find an $a_{1}$ transition on top, since $e_{2}=a_{1} \cup \mathbb{Z}_{2}$, where $\mathbb{Z}_{2}$ is an abuse of notation to denote the 'fat point' non-reduced algebraic scheme [57]. The following Coulomb branch modulus can be opened up:

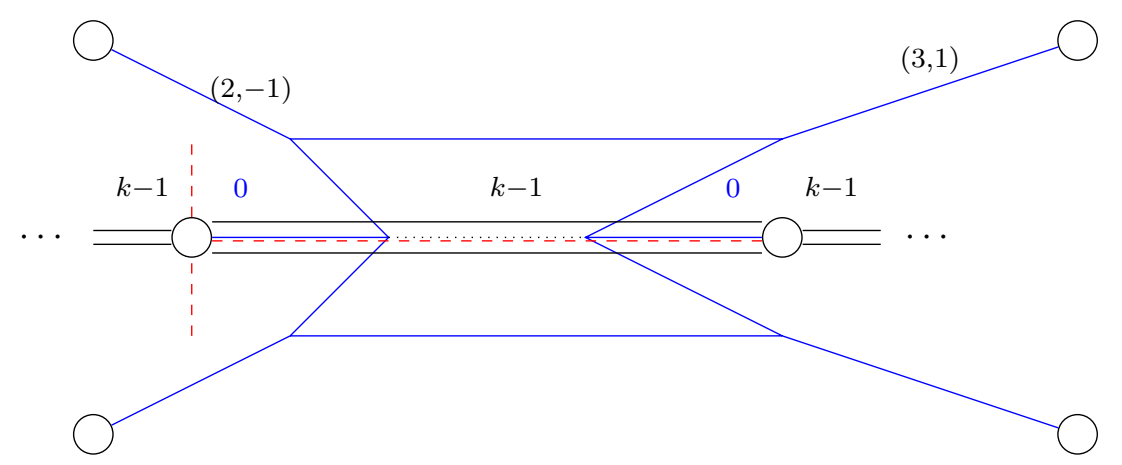

where the blue brane subweb corresponds to the $E_{2}$ configuration (2.53), with magnetic quiver (2.54). The magnetic quiver for (5.21) is

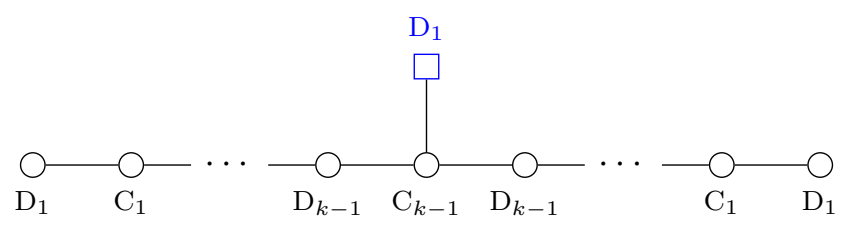


The Hasse diagram is

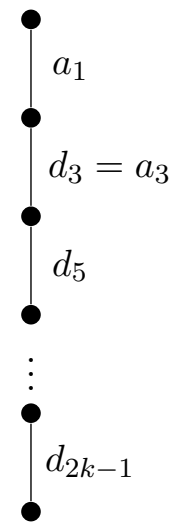

which matches the Hasse diagram [70, table 26] obtained from the unitary magnetic quiver (4.16). For $k=1$, the Hasse diagram reduces to a single $e_{2}$ transition.

\section{$5.8 E_{1}$ family}

Next, for $\operatorname{Sp}(k)$ with $N_{f}=2 k-2$ flavours at infinite coupling, the brane web is provided in (3.18). To open up the minimal amount of Coulomb branch directions, one finds

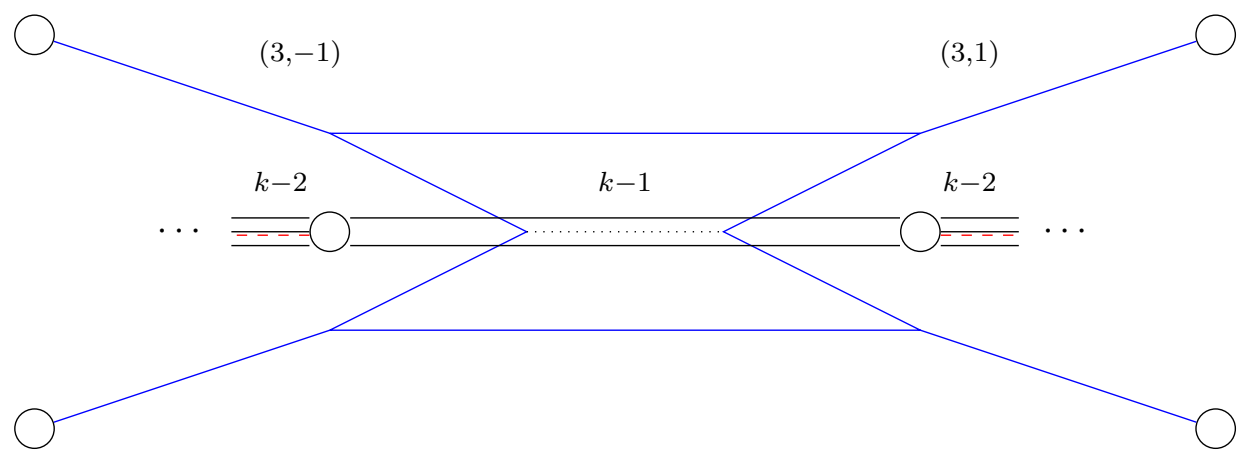

where the blue brane subweb corresponds to the $E_{1}$ configuration (2.58), with magnetic quiver (2.59). The magnetic quiver for (5.24) is

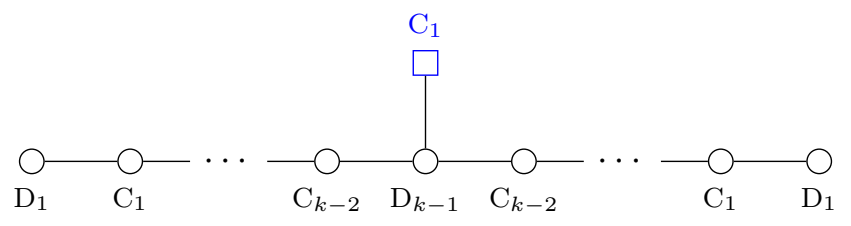

However, the problem lies in identifying the second cone from the brane web with O5 plane such that only a part of the Hasse diagram is visible. Nevertheless, the missing transition, denoted in red, can be seen from the $\mathrm{O}^{-}$construction of section 4 . Then, the full Hasse 
diagram is

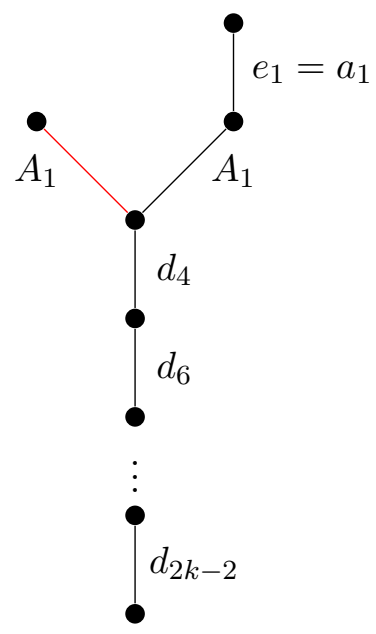

which matches the Hasse diagram [70, table 6$]$ obtained from the unitary magnetic quivers $(4.14 \mathrm{a})$ and $(4.14 \mathrm{~b})$. The red transition stems from (4.14a). Note that the two $A_{1}$ transitions above the $d_{4}$ can be viewed as a $d_{2}=A_{1} \cup A_{1}$. For $k=2$ the Hasse diagram consists only of $A_{1} \cup A_{1}$ with the $e_{1}$ transition on one cone.

\section{$5.9 E_{\leq 0}$ families}

For the top transitions in the Higgs branch Hasse diagram of $\operatorname{Sp}(k)$ with $N_{f}<2 k-2$ fundamental flavours at infinite coupling one does a similar transition on the brane web, (3.18) or (3.24), as for the $E_{2}$ or $E_{1}$ family. Thus, one finds a $A_{k-N_{f} / 2}$ transition for $N_{f}$ even and $A_{k-\left(N_{f}-1\right) / 2}$ transition for $N_{f}$ odd. In the case of $N_{f}$ even, only one cone is visible, the second cone is denoted in red as above. In total, one obtains the following Hasse diagram:

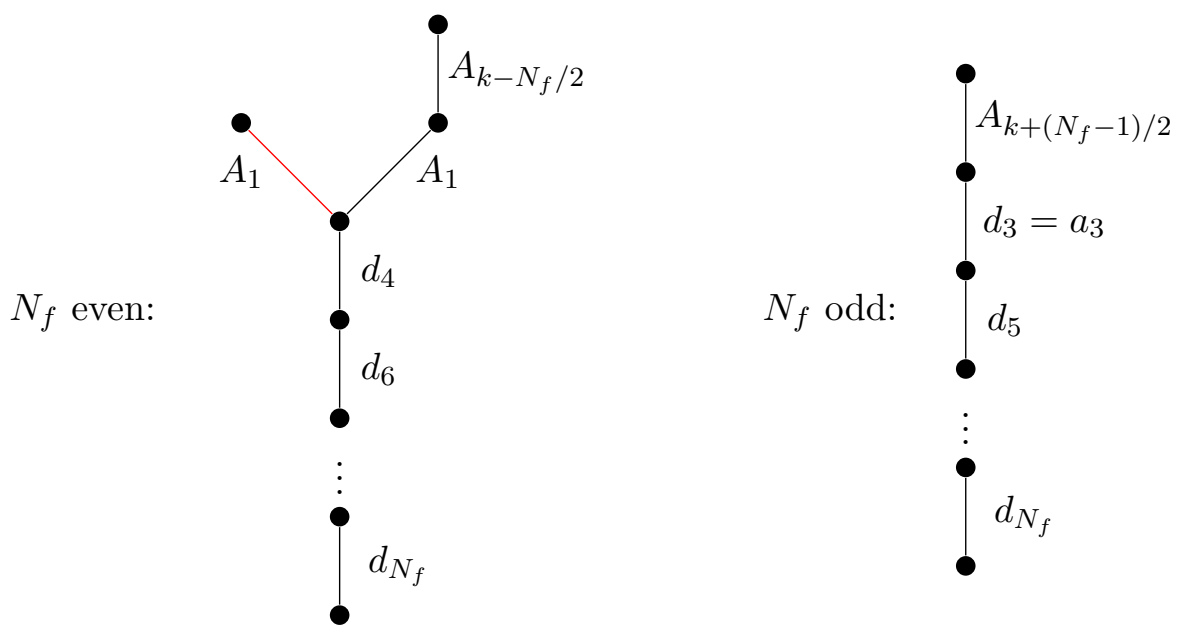

which matches the Hasse diagram [70, table 6] obtained from the unitary magnetic quivers (4.14a) and (4.14b) for $N_{f}$ even, and (4.16) for $N_{f}$ odd.

\section{Linear orthosymplectic quiver theories}

Instead of a theory with a single gauge group, one can construct quiver gauge theories with alternating orthogonal and symplectic gauge groups. Consider, for instance, the following 
5-brane web

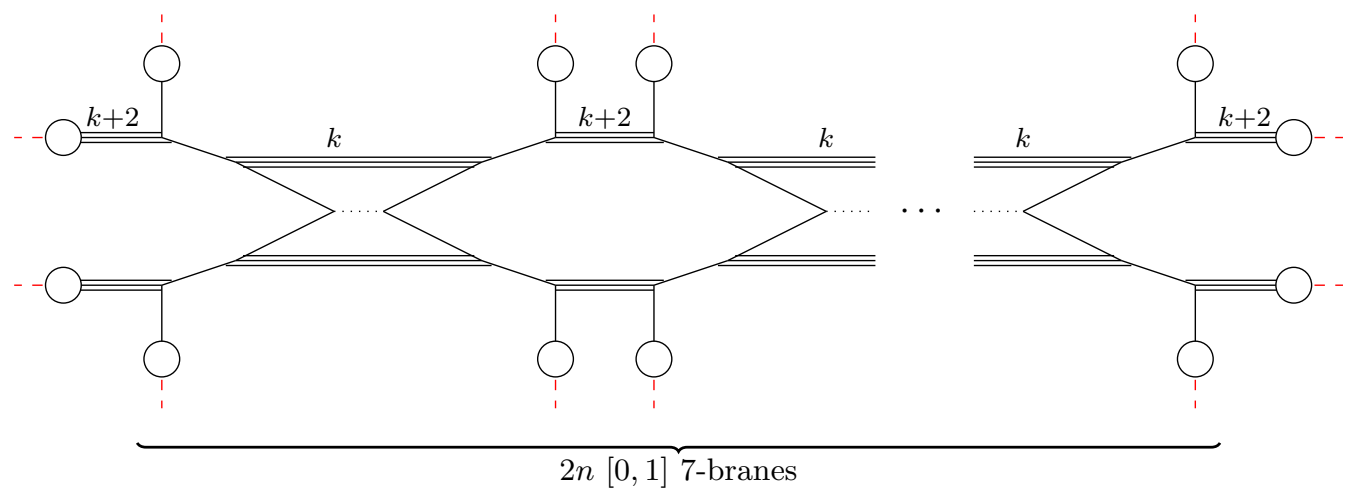

which gives rise to the following $5 \mathrm{~d} \mathcal{N}=1$ quiver gauge theory

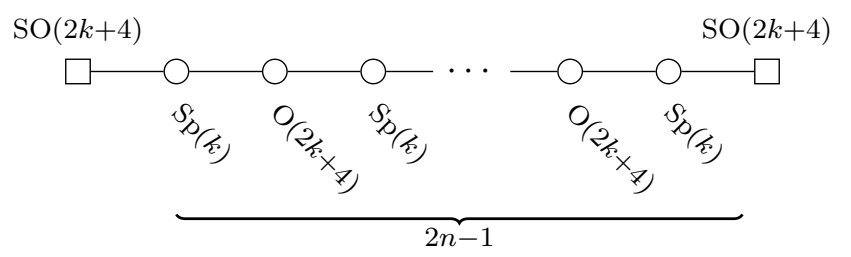

The theory (6.2) gives rise to various different Higgs branches. Given that there are $2 n-1$ gauge couplings, any of these can either be finite or infinite. In the following, the finite coupling phase, some intermediate, and the fixed point phase are detailed.

Finite coupling. Starting from the brane web (6.1), the finite coupling Higgs branch phase is accessible as detailed above: aligning flavour and gauge branes on the orientifold, such that the half $[1,0]$-branes merge with their mirror images. The resulting physical $[1,0]$ 7-branes can split along the orientifold and one obtains

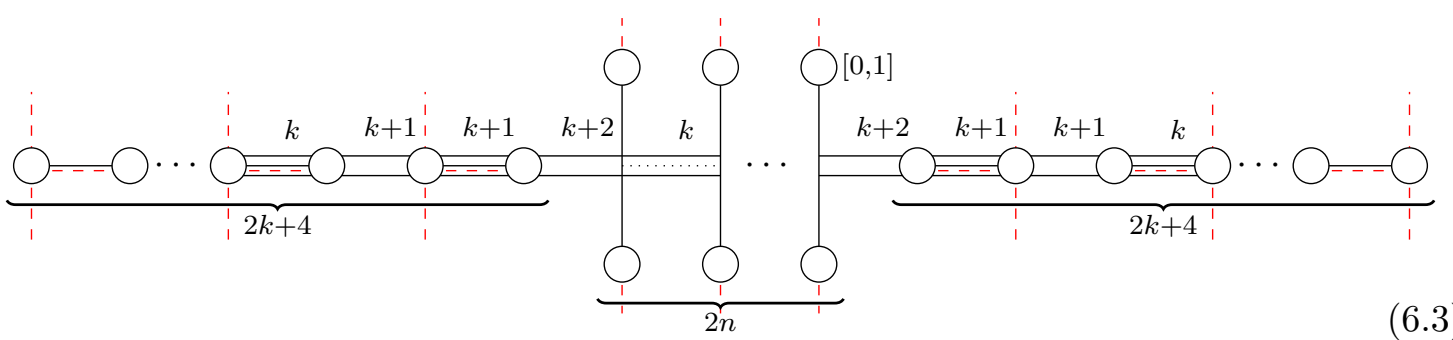

The classical Higgs branch of (6.2) is of dimension

$$
\begin{aligned}
\operatorname{dim}_{\mathbb{H}} \mathcal{H}(6.2) & =n_{h}-n_{v}=k(2 k+7)=\operatorname{dim} \mathrm{O}(2 k+4)-\operatorname{dim} \mathrm{O}(4), \\
n_{h} & =\frac{1}{2} \cdot 2 k \cdot(2 k+4) \cdot 2 n, \\
n_{v} & =n \cdot \operatorname{dim} \mathrm{Sp}(k)+(n-1) \cdot(\operatorname{dim} \mathrm{O}(2 k+4)-\operatorname{dim} \mathrm{O}(4)) .
\end{aligned}
$$

Note that there is no complete Higgsing for the orthogonal gauge groups, analogous to the $6 \mathrm{~d}$ scenario of [61, section 2.4]. 
In order to find a suitable configuration to read off the magnetic quiver, one moves the left and right outermost $(0,1) 5$-branes through three half 7 -branes each such that the brane web becomes

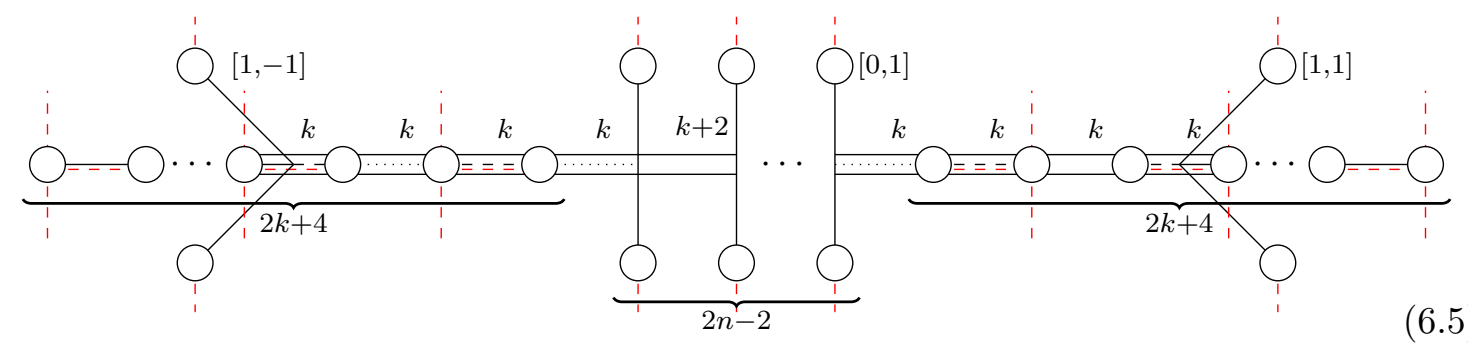

The central segments deserve some comments: there are $k$ physical D5 branes that are freely moving subwebs between 7 -branes. In addition, there are pairs of $(0,1)$ 5-branes that have 2 extra D5 suspended in between; these are interpreted as residual gauge branes. In order words, these are subwebs that remain on the Coulomb branch and, as such, they contribute as flavour to the magnetic quiver

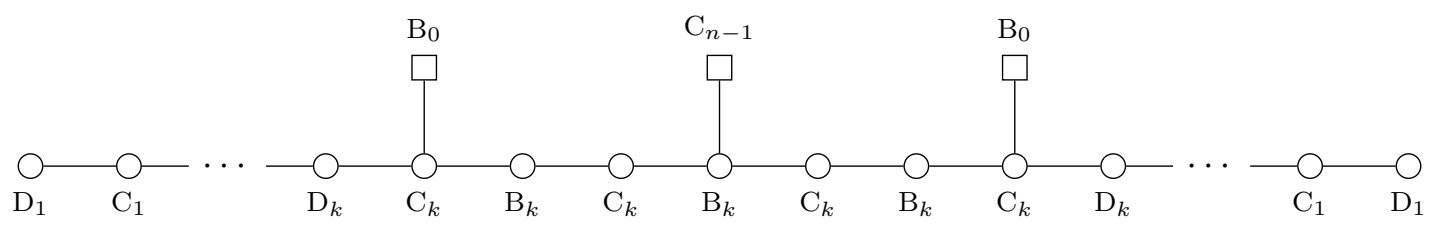

for which one can compute

$$
\begin{aligned}
\operatorname{dim}_{\mathbb{H}} \mathcal{C}(6.6) & =\operatorname{dim} \mathrm{O}(2 k+4)-\operatorname{dim} \mathrm{O}(4), \\
G & =\operatorname{SO}(2 k+4) \times \mathrm{SO}(2 k+4) .
\end{aligned}
$$

The global symmetry is a product because the central $\mathrm{B}_{k}$ node is not balanced, see appendix A.3. Compared to the classical Higgs branch of (6.2), the magnetic quiver (6.6) correctly reproduces dimension (6.4) and the non-abelian global symmetry, but the way the magnetic quiver is read from the brane system is not sensitive to the possibility of multiple cones in the Higgs branch. Moreover, note that this magnetic quiver is similar to the $6 \mathrm{~d}$ case [61, section 2.4].

One infinite gauge coupling. Starting from the finite coupling phase, one can now inquire the effects of turning a single gauge coupling to infinity. Inspecting the brane web (6.5), one notices there are two potentially different transitions that make two $(0,1)$ 5-brane subwebs become coincident.

(i) Two $(0,1) 5$-brane subwebs on an $\mathrm{O}^{+}$plane, where the $k$ physical D5 branes are suspended between 7 -branes.

(ii) Two $(0,1) 5$-brane subwebs on an $\mathrm{O}^{-}$plane, where two out of the $k+2$ physical D5 branes are suspended between the NS5 branes, forming the residual $O(4)$ gauge group. 
To begin with, consider the first case, i.e. the brane web is

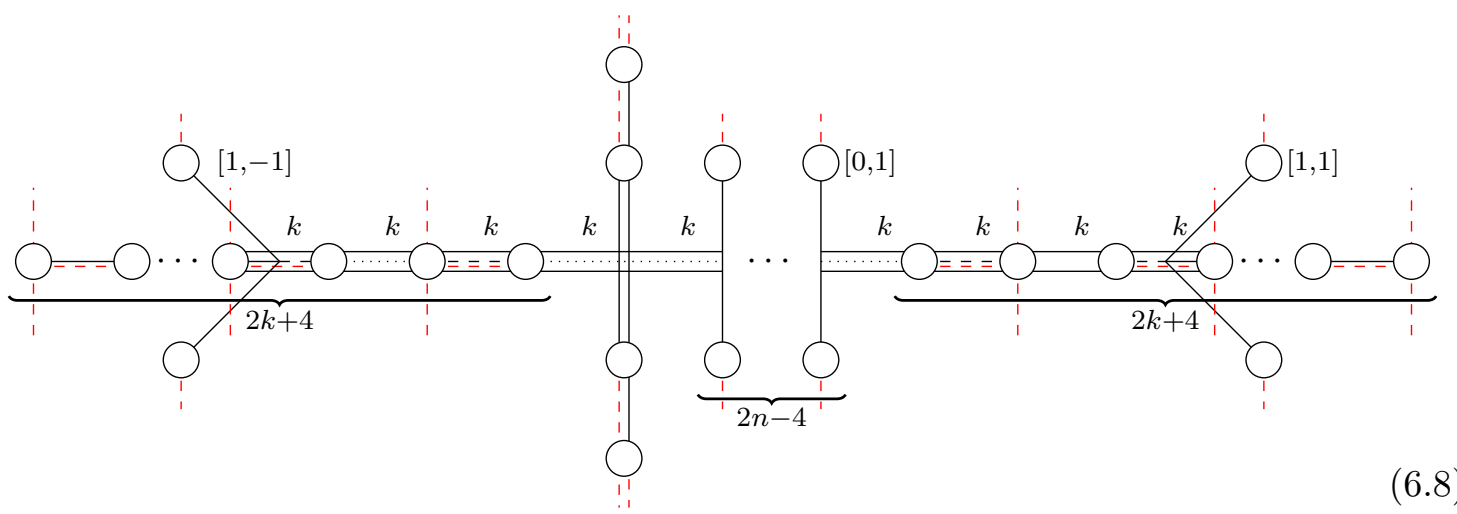

and the change of the magnetic quiver is apparent. The $(0,1) 5$-branes have entered the Higgs branch and are freely moving subwebs suspended between 7-branes. Thus, (6.6) changes to

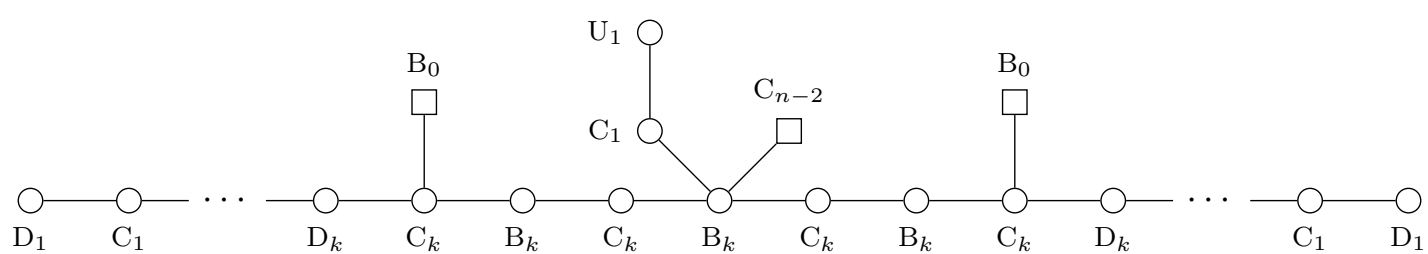

and the dimension has increased

$$
\operatorname{dim}_{\mathbb{H}} \mathcal{C}(6.9)=2+\operatorname{dim} \mathrm{O}(2 k+4)-\operatorname{dim} \mathrm{O}(4) .
$$

In terms of the electric theory, a residual $\mathrm{O}(4)$ gauge symmetry vanished.

Considering now the second possibility, the brane web (6.5) changes to

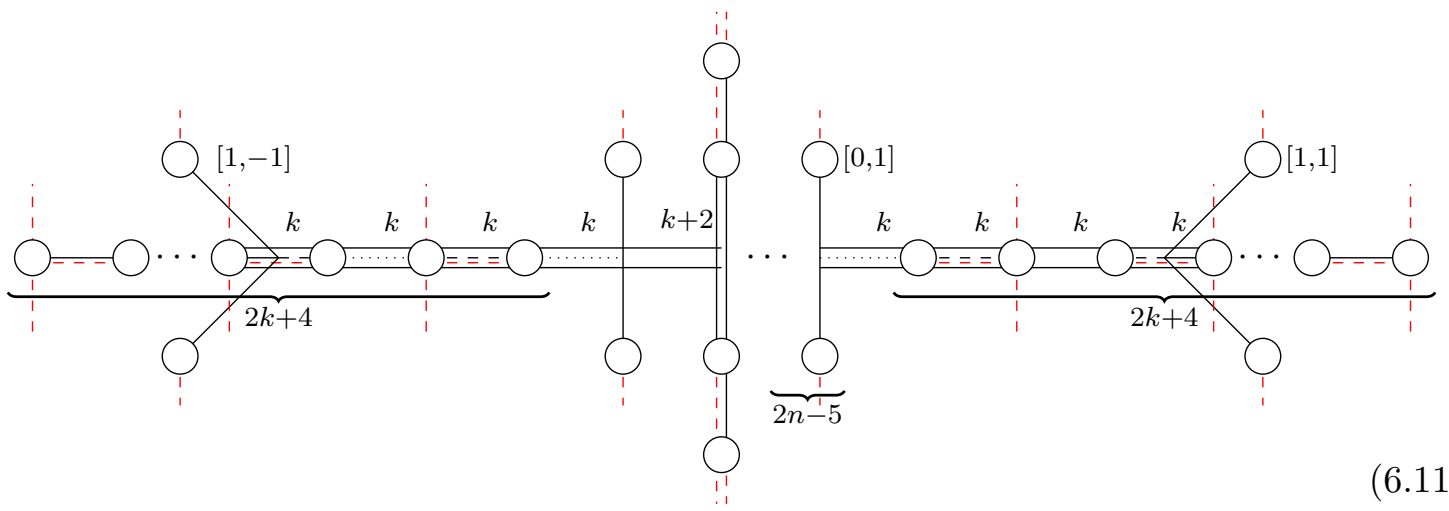

such that the new Higgs branch degrees of freedom are suspended on an O5 interval with a residual $\mathrm{O}(4)$ gauge symmetry. Analogous to the proposal of [61, section 2.4], the contribution of this gauge symmetry is argued to be a flavour in the magnetic quiver which 
reads as

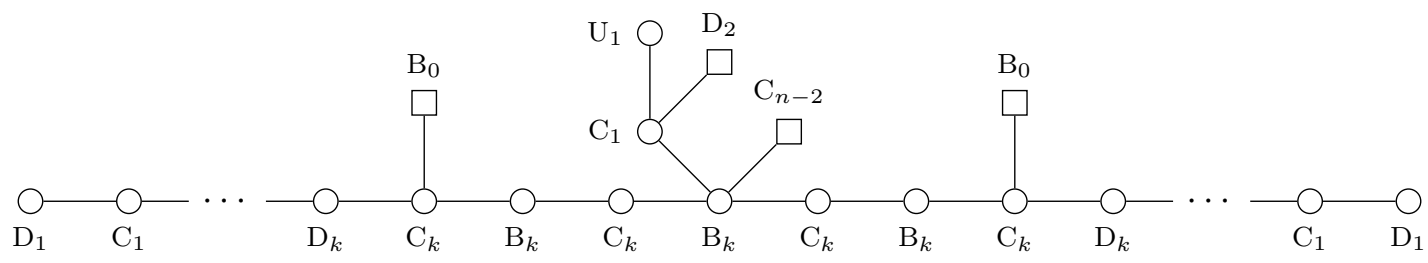

and the dimension has increased

$$
\operatorname{dim}_{\mathbb{H}} \mathcal{C}(6.12)=2+\operatorname{dim} \mathrm{O}(2 k+4)-\operatorname{dim} \mathrm{O}(4) .
$$

As in the first case (6.8), the web (6.11) clearly displays the loss of an $\mathrm{O}(4)$ residual gauge symmetry.

Several infinite couplings. Repeatedly taking couplings to infinite, in the form of (6.8) or (6.11), and assuming that at most two NS5 branes become coincident, one obtains a generalisation of the notion of bouquet for a magnetic quiver [60,61, 66, 98, 99]. For instance, allowing $n_{1}$ transitions of type (6.9) and $n_{2}$ transitions of type (6.12) such that $n_{1}+n_{2} \leq n-1$ the magnetic quiver reads

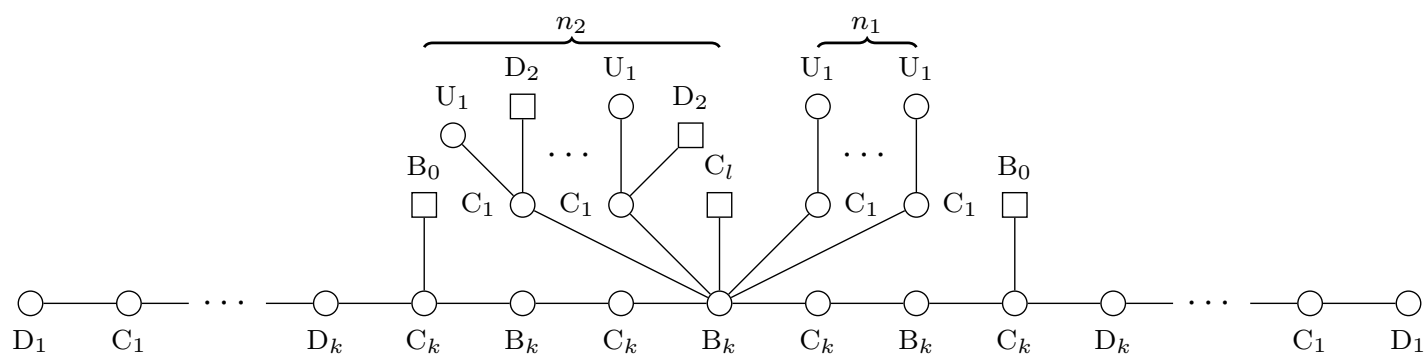

with $l=n-1-n_{1}-n_{2} \geq 0$.

So far, the assumption has been to have at most two coinciding NS5 branes. In order to move towards the fixed point of (6.2) one needs to coincide all of the NS5 simultaneously. As an exemplary case, consider (6.5) and make the first $2 j$ half NS5 branes on the left- 
hand-side of the central segment coincident. Then the brane web becomes

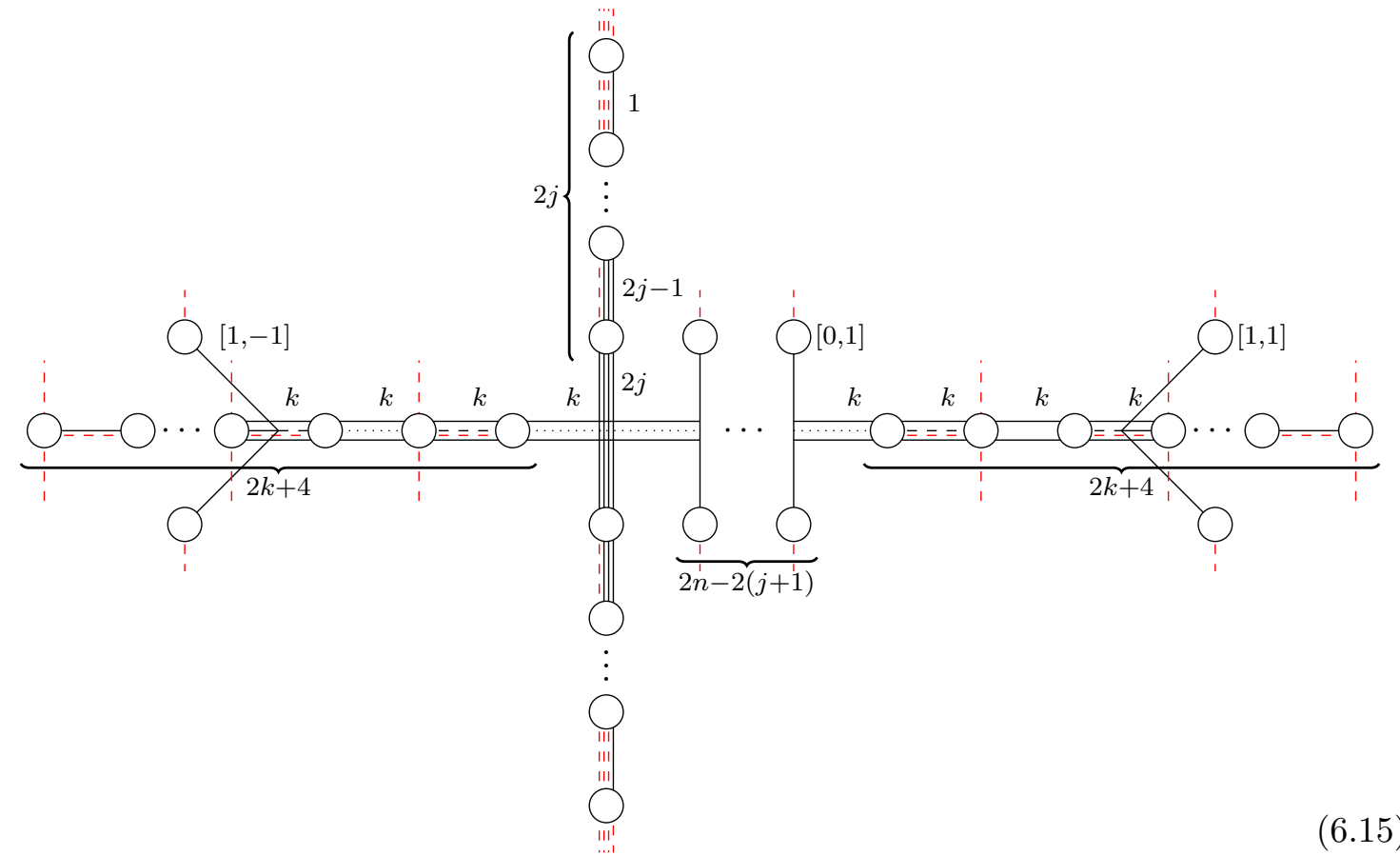

such that one now has to explain how to read off the magnetic quiver. The D5 branes suspended between half $[1,0]$ 7-branes along the orientifold are no conceptual challenge, due to the discussion above. In contrast, the half NS5 branes between half $[0,1] 7$-branes behave differently. The set of $2 j$ identical $(0,1)$ 5-brane subwebs suspended between a half 7 -brane and its mirror image is affected by the orientifold projection, because the NS5 branes cross the $\mathrm{O} 5$ plane. Hence, this set of identical subwebs contributes a $\mathrm{C}_{j}$ magnetic gauge node. In the adjacent segments, there is always a set of $m$ identical NS5 branes suspended between two half [0,1] 7-branes. Note, however, that these NS5 branes do not cross the orientifold and, therefore, the associated magnetic gauge node is a $\mathrm{U}(m)$. Following [24], the unitary nodes are connected by magnetic bi-fundamental hypermultiplets. In summary, the magnetic quiver for (6.15) reads

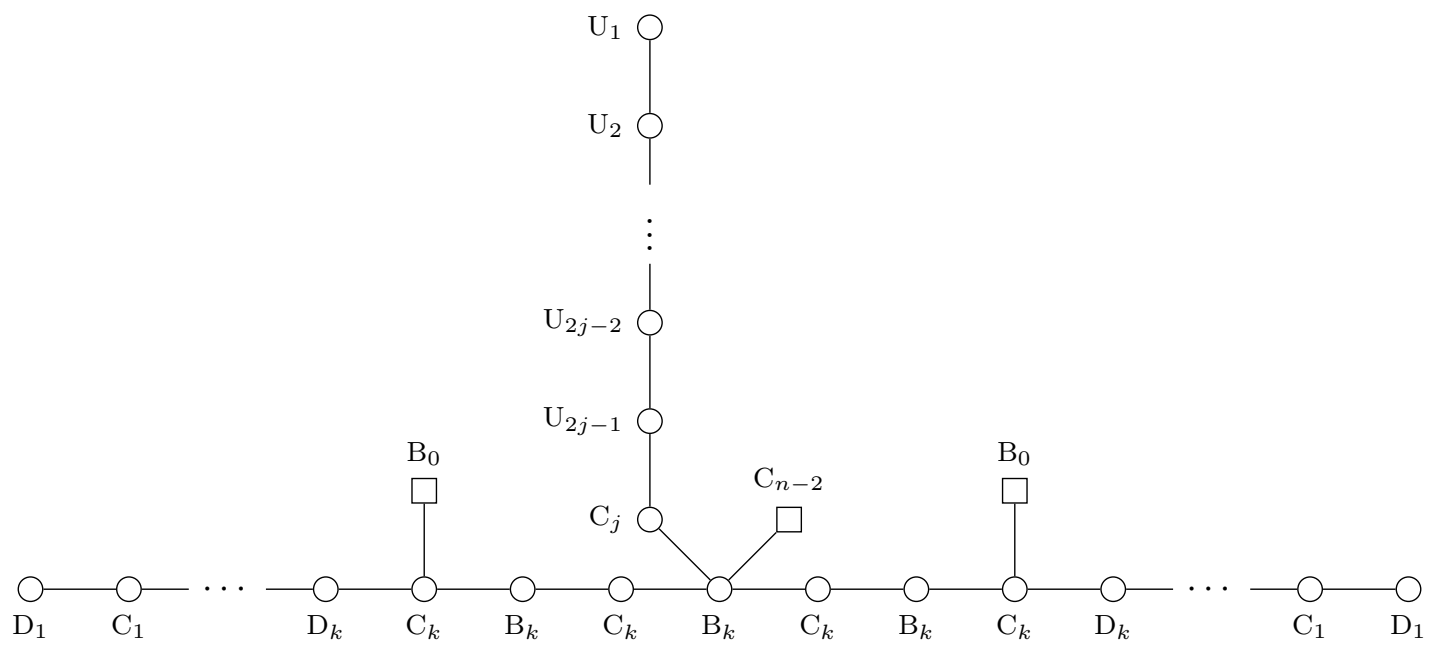


and the dimension is increased as

$$
\operatorname{dim}_{\mathbb{H}} \mathcal{C}(6.16)=2 j^{2}+\operatorname{dim} \mathrm{O}(2 k+4)-\operatorname{dim} \mathrm{O}(4) .
$$

Analogously, if instead one coincides $2 j$ half NS5 branes over a segment with a residual $\mathrm{O}(4)$ gauge symmetry, the only change compared to (6.16) lies in an additional $\mathrm{D}_{4}$ flavour node for the $\mathrm{C}_{j}$ gauge node, similar to (6.12).

Infinite coupling. To transfer the finite coupling brane web (6.5) into the fixed point phase, the various $(0,1) 5$-branes, i.e. NS5 branes, have to be made coincident. So far, it has been demonstrated how to make at most $2 n-2$ half NS5 brane coincident starting from the brane web (6.5). The process proceeds via the two fundamental transitions (6.8), (6.11) via various intermediate phases (6.15). However, to reach the fixed point phase, the two outermost $(1, \pm 1) 5$-branes need to be transitioned to the central segment again, i.e. one starts from (6.3). Next, merging all the half NS5 branes pairwise, the brane web becomes

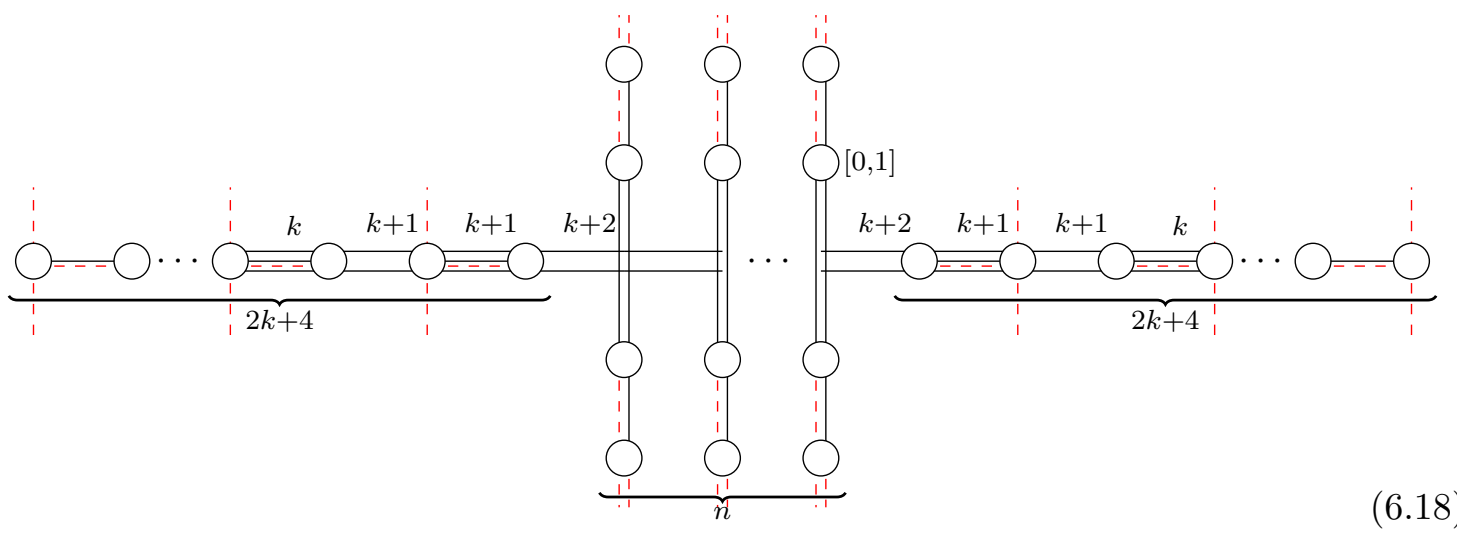

such that the magnetic quiver is straightforwardly read off

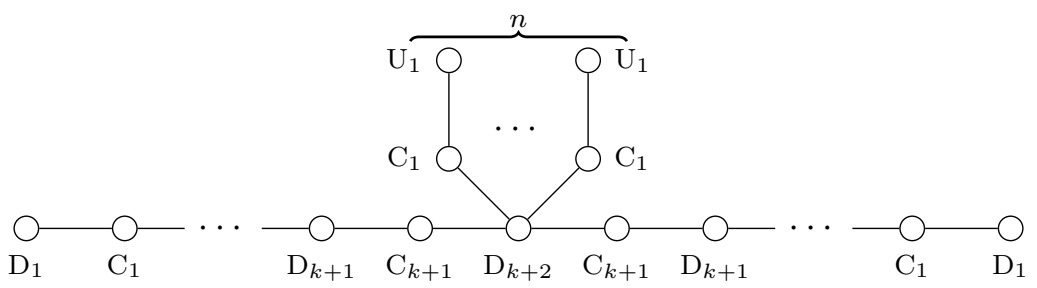

with a Coulomb branch of dimension

$$
\operatorname{dim}_{\mathbb{H}} \mathcal{C}(6.19)=2 n+6+\operatorname{dim} \mathrm{O}(2 k+4)-\operatorname{dim} \mathrm{O}(4) .
$$

Note that the moduli space dimension of (6.19) is increased by two sources: (i) each pair of coincident half NS5 branes yields 2 Higgs branch degrees of freedom, and (ii) moving the outermost $(1, \pm 1)$-branes from (6.5) into the central segment leads to another 6 Higgs branch degrees of freedom due to D5 branes suspended between 7-branes along the orientifold plane. 
Finally, one can make all NS5 branes coincident. Analogously to $(6.15)$, the $[0,1] 7$ branes can be vertically displaced because the NS5 branes can be split between them. As a consequence, the brane web becomes

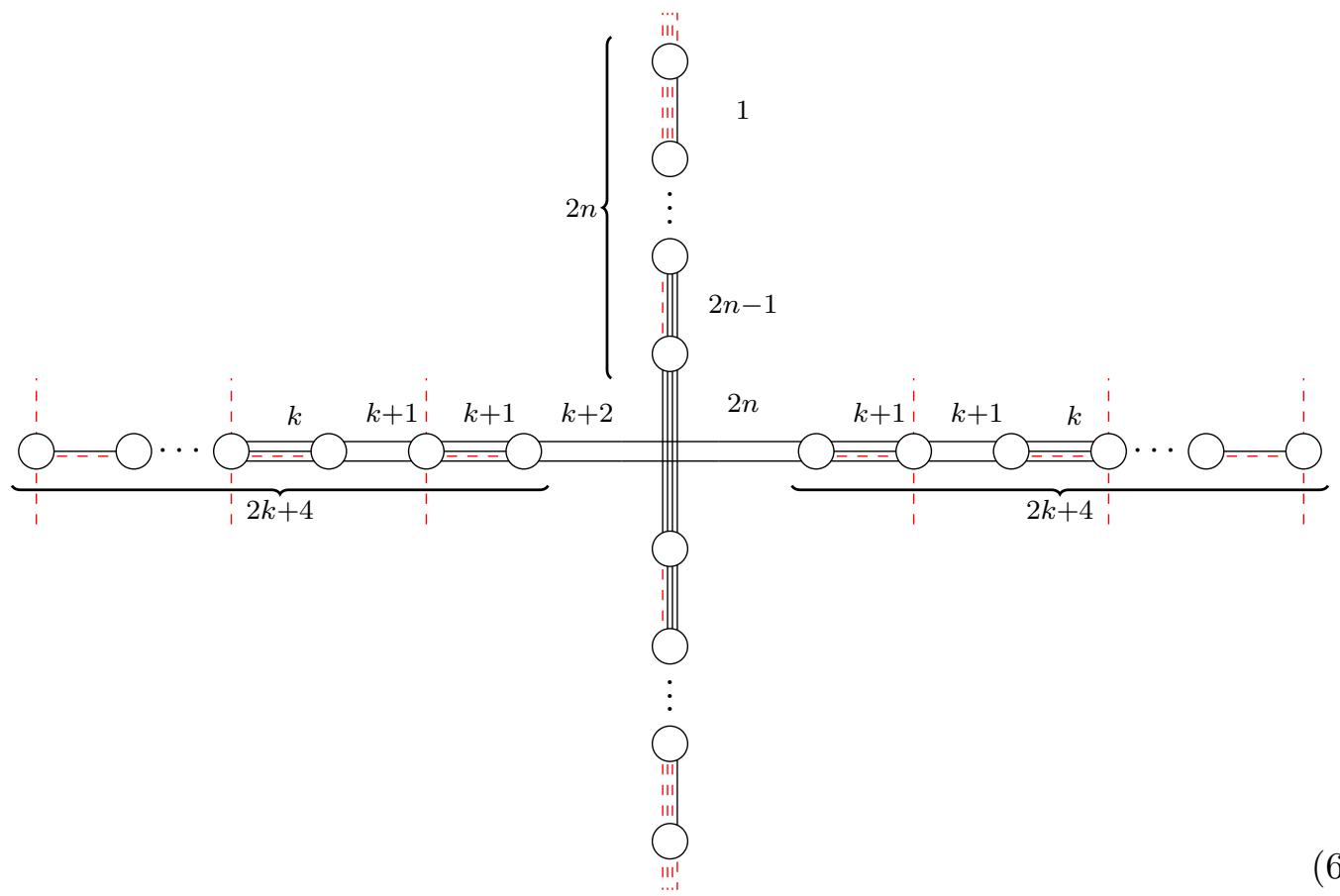

The 5-brane webs suspended between 7-branes on the orientifold are no conceptual challenge, due to the discussions above. The $(0,1) 5$-branes, in contrast, need to be examined in detail. The first $2 n(0,1)$ branes that go through the $\mathrm{O} 5$ plane contribute a $\mathrm{C}_{n}$ magnetic gauge node, because the orientifold projection acts on these subwebs. The subsequent $j$ copies of identical $(0,1) 5$-branes between adjacent half 7 -branes are not affected by the orientifold, because they are away from the plane. Hence, these stacks of identical subwebs contribute magnetic $\mathrm{U}(j)$ gauge nodes. Put differently, away from the orientifold, the rules of [24] apply without modifications. Putting all the pieces together, the magnetic quiver reads

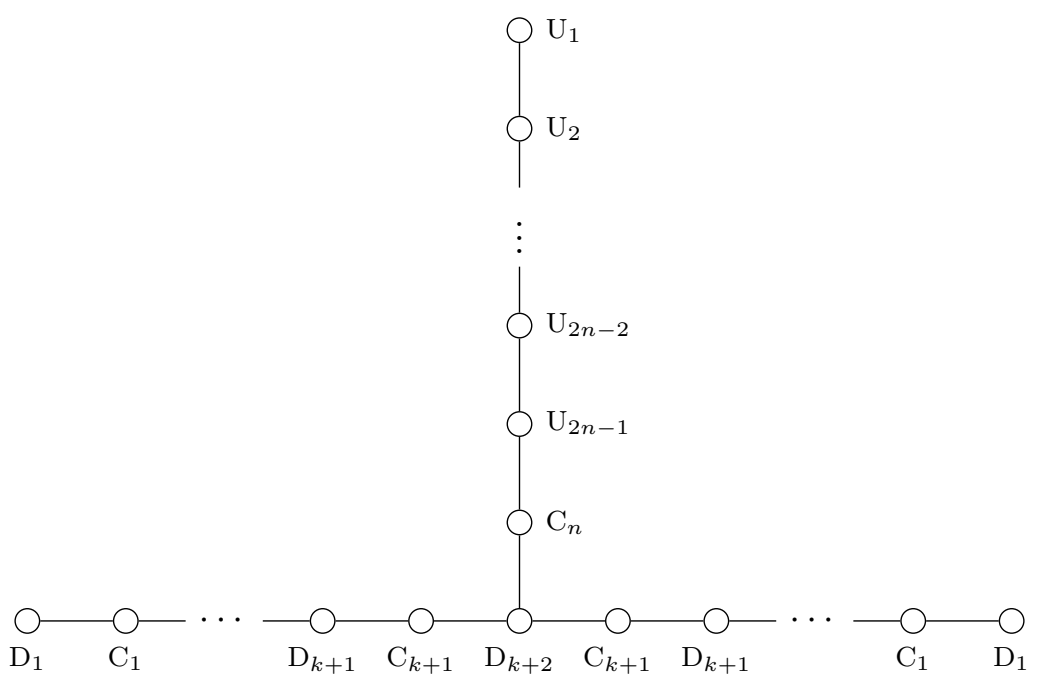


and its Coulomb branch dimension is computed to be

$$
\operatorname{dim}_{\mathbb{H}} \mathcal{C}(6.22)=2 n^{2}+6+\operatorname{dim} \mathrm{O}(2 k+4)-\operatorname{dim} \mathrm{O}(4) .
$$

Compare to the finite coupling magnetic quiver (6.6), the moduli spaces has grown in dimension by $2 n^{2}+6$ quaternionic units. The increase can be traced back to two different origins, similarly to (6.19): (i) the 6 additional Higgs branch directions originate from D5 branes suspended between 7 -branes along the O5 plane; while, (ii) the $2 n^{2}$ new moduli originate from the coincident NS5 branes.

Hasse diagram. For the quiver theories (6.2) one could, in principle, detail the Hasse diagrams as well. Inspecting the infinite coupling magnetic quiver (6.22) shows that the challenge lies in the unitary-orthosymplectic quiver with a large number of unitary nodes. From the insights gained in section 2, it is apparent that the first minimal transitions that open up a Coulomb branch direction are necessarily $e_{7}$ or $e_{8}$ transitions. Thereafter, the number of possible transitions is quite involved and there is no clear gain of attempting to derive the entire Hasse diagram.

\section{Conclusion}

In this work, the Higgs branches of $5 \mathrm{~d} \mathcal{N}=1$ theories with symplectic or orthogonal gauge groups and fundamental matter are investigated at finite and infinite gauge coupling. Based on the 5-brane web realisations in the presence of $\mathrm{O} 5$ orientifold planes, the key technique for this study is the use of magnetic quivers. More generally, the techniques presented here open a window on the Higgs branch of any 5d SCFT that can be engineered via brane webs involving $(p, q)$ 5-branes and $\mathrm{O} 5$ planes, which potentially includes theories with exceptional gauge algebra $G_{2}$.

Compared to the magnetic quiver proposal [24] for brane webs without O5 planes, the conceptual challenge is the inclusion of the orientifolds. As detailed in section 2, the proposed magnetic orientifolds of [61] do consistently reproduce known Higgs branch geometries at finite coupling and some infinite coupling cases. In addition, an unprecedented phenomenon appeared at infinite coupling: due to additional Higgs branch directions the corresponding magnetic quivers become a combination of orthosymplectic and unitary quivers. Therefore, magnetic orientifolds need to be supplemented by unitary magnetic gauge multiplets and corresponding matter fields, in order to address all Higgs branch phases.

In section 3, the magnetic quiver proposal has been applied to $\operatorname{Sp}(k)$ gauge theories with $N_{f} \leq 2 k+5$ flavours. The finite coupling Higgs branches are all described by orthosymplectic magnetic quivers, which correctly reproduce moduli space dimensions and the non-abelian part of the global symmetry. A further matching between the Higgs branch Hilbert series and magnetic quiver Hilbert series is detailed in a companion paper [80]. For infinite gauge coupling, the magnetic quivers do match the moduli space dimensions, in agreement with the additional Higgs branch directions at the fixed point found in [10]. Further Hilbert series details will be presented in [80]. Moreover, the infinite coupling results fall into generalised $E_{n}$ classes (for $-\infty<n \leq 8$ ), summarised in tables $1,2,3$ 
and 4. It is important to note that when the finite coupling Higgs branch consists of two identical cones (i.e. when $N_{f} \leq 2 k$ even), from the O5 construction only cone can be seen. At infinite coupling only one cone gets enhanced, which is the cone obtained from the O5 construction.

Besides $\mathrm{O}^{+}$planes, one may engineer $\mathrm{Sp}(k)$ theories via $\mathrm{O}^{-}$planes as detailed in section 4. Since an $\mathrm{O}^{-}$plane can be resolved into a pair of $[1, \pm 1] 7$-branes, one can analyse these cases with the techniques developed in [24]. Interestingly, this approach yields unitary magnetic quiver for the same Higgs branches as discussed in section 3 . On the one hand, the unitary magnetic quivers allow to immediately verify dimension and global symmetry of the various Higgs branches. These agree with known symmetry enhancement results at the fixed point. On the other hand, one has to confirm that the unitary-orthosymplectic quiver from the $\mathrm{O} 5$ plane construction and the unitary magnetic quiver from the $\mathrm{O}^{-}$construction yield the same moduli space. One step further towards a verification is subject of a companion paper [80]. Most importantly, this provides different realisations for the same Higgs branches, as summarised in table 1.

Finally, the validity of the proposed modification for magnetic quivers from 5-branes on O5 planes is further underpinned by deriving the associated Hasse diagrams. As detailed in section 5 for the generalised $E_{n}$ families (for $-\infty<n \leq 8$ ), the Hasse diagrams are derived starting from the 5-brane web with $\mathrm{O} 5$ at infinite coupling and then opening up a minimal amount of Coulomb branch directions. The transitions found are all exceptional minimal nilpotent orbit closures (or the appropriate substitution for $n \leq 3$ ), because the transitions are realised by the brane configurations of section 2.3. Thereafter, the remaining magnetic quivers are all framed orthosymplectic quivers, such that conventional quiver subtraction suffices to completely determine the Hasse diagram. A non-trivial consistency check is provided by verifying that the Hasse diagrams from the unitary magnetic quivers of section 4 agree with those from the unitary-orthosymplectic quivers.

As a next more complicated example, section 6 deals with $5 \mathrm{~d} \mathcal{N}=1$ linear orthosymplectic quiver gauge theories. In contrast to the single gauge group cases, the quiver theories admit a multitude of Higgs branch phases, depending on which subset of gauge couplings has been taking to infinity. In particular, there exist two types of infinite coupling transitions (6.9) and (6.12) that are expected to open up two new Higgs branch dimensions. One notes the close analogy to the $D_{4}$ transition of [61]. However, the $5 \mathrm{~d}$ setting of a generalised bouquet of type

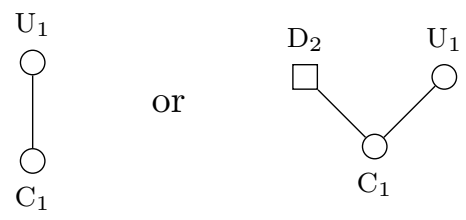

is not comparable to the discrete gauge phenomenon $[66,98,99]$. If the relevant NS5 branes become coincident, the resulting magnetic quiver is obtained by replacing the bouquet by a longer unitary tail. 


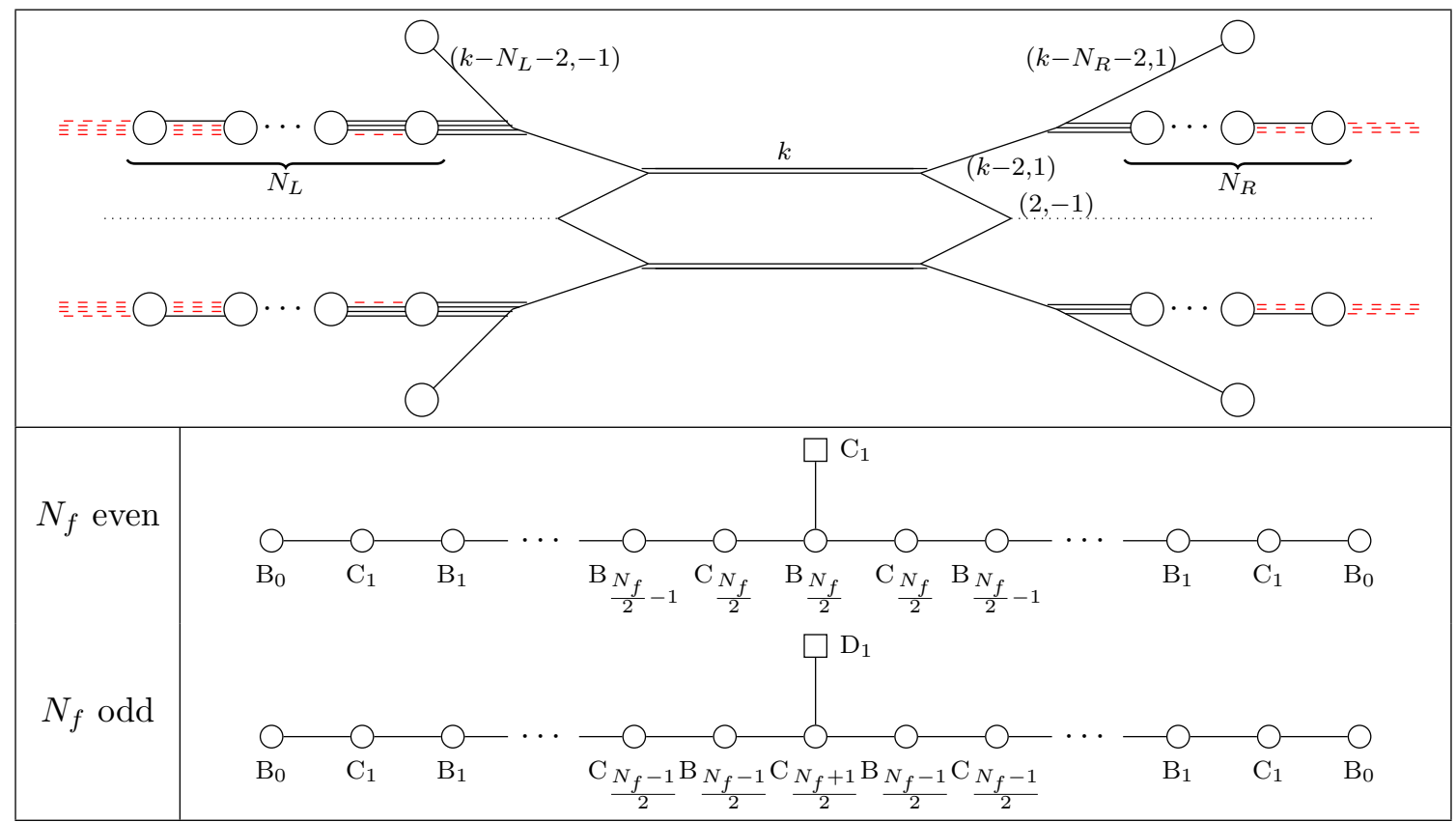

Table 9. Brane web for $\mathrm{O}(2 k)$ with $N_{f}=N_{L}+N_{R}$ flavours. On a generic point of the Higgs branch $\mathrm{O}(2 k)$ with $N_{f}$ flavours is broken to pure $\mathrm{O}\left(2 k-N_{f}\right)$ gauge theory. The magnetic quivers describe then a moduli space of quaternionic dimension $\frac{1}{2} N_{f}\left(N_{f}+1\right)$.

Further predictions. In addition to 5 -brane webs on $\mathrm{O}^{+}$planes, one may equally well study configurations involving $\mathrm{O} 5^{-}$and $\widetilde{\mathrm{O}} 5^{-}$plane, which low-energy descriptions in terms of $\mathrm{O}(k)$ gauge theories. For $\mathrm{O}^{-}$planes the constraints on the number of flavours $N_{f} \leq 2 k-4$ for a non-trivial interaction fixed point are well-known [3, 8]. In addition, it has been argued in [9, section 3.1] that $N_{f}=2 k-3$ also leads to an interacting $5 \mathrm{~d}$ fixed point. The relevant brane configuration is depicted in table 9. Analysing the Higgs branch via the conjecture 1 and a generalisation of observation 1, leads to the magnetic quivers for finite coupling in table 9 and infinite coupling in table 11. Likewise, brane webs with $\widetilde{\mathrm{O}}^{-}$ planes can be reached via Higgsing the brane web of $\mathrm{O}(2 k+2)$ with $N_{f}+1$ fundamental flavours to the web corresponding to $\mathrm{O}(2 k+1)$ with $N_{f}$ flavours, see table 10 . The brane web yields the constraint $N_{f} \leq 2 k-3$, while [9] showed the existence of another non-trivial fixed point for $N_{f}=2 k-2$. The finite coupling magnetic quivers are summarised in table 10, while the infinite coupling magnetic quivers are shown in table 12.

In contrast to the symplectic case, the orthogonal gauge theories do not admit complete Higgsing such that already the finite coupling Higgs branch represents a computational challenge. Here, the magnetic quivers derived provide a prediction for the Higgs branches at all values of the coupling. So far, the magnetic quivers do consistently reproduce the expected dimensions. Similar to the open puzzles in $6 \mathrm{~d}$ theories with orthogonal gauge groups [61], a more detailed analysis is left for future research.

Outlook. Having considered all allowed numbers of flavours for $\operatorname{Sp}(k)$ theories, one should have observed instances of multiple cones. As known from [94], for $N_{f}=2 k$ the 


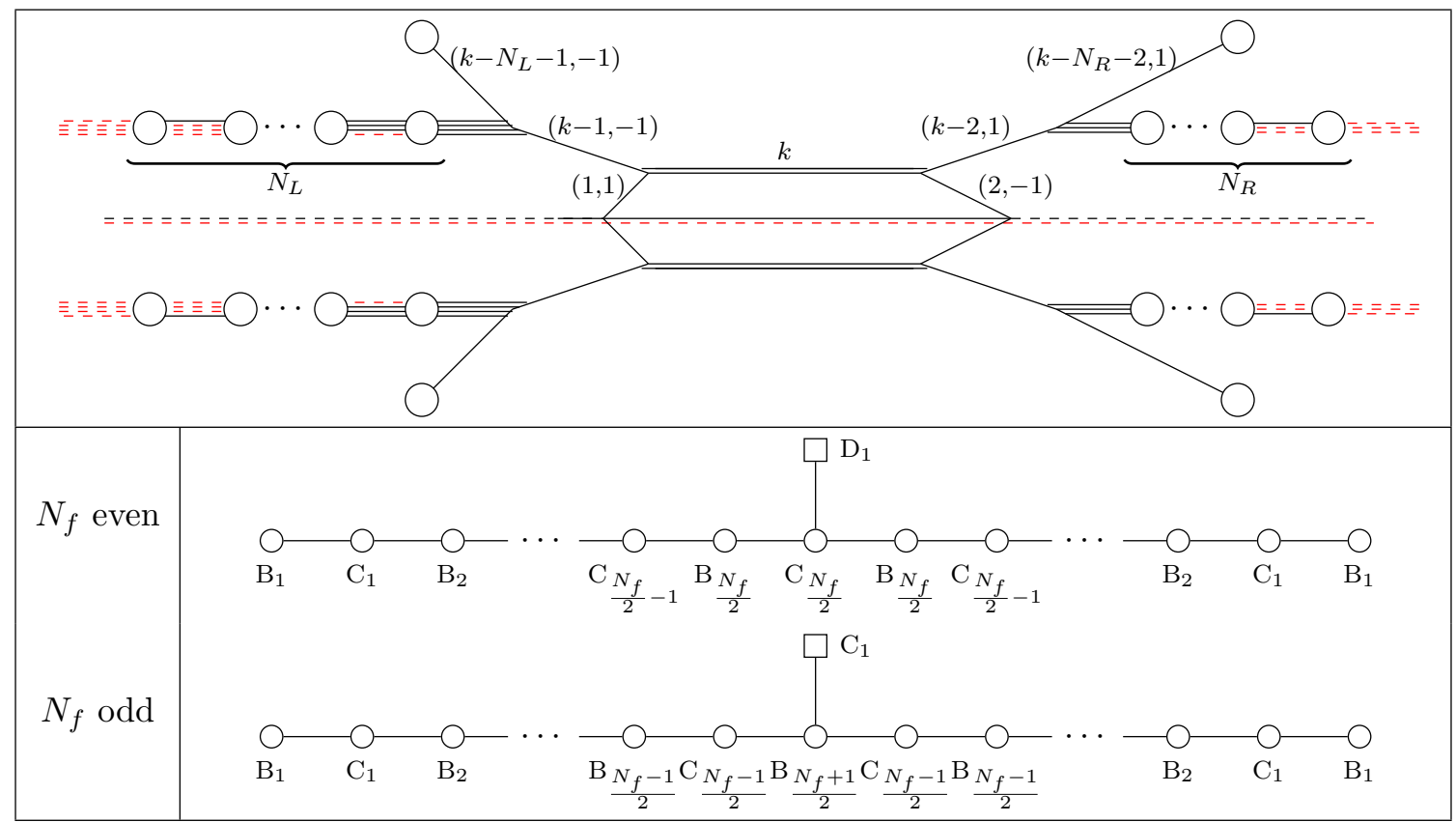

Table 10. Brane web for $\mathrm{O}(2 k+1)$ with $N_{f}=N_{L}+N_{R}$ flavours. On a generic point of the Higgs branch $\mathrm{O}(2 k+1)$ with $N_{f}$ flavours is broken to pure $\mathrm{O}\left(2 k+1-N_{f}\right)$ gauge theory. The magnetic quivers describe then a moduli space of quaternionic dimension $\frac{1}{2} N_{f}\left(N_{f}+1\right)$.

Higgs branch is a union of two cones. In terms of the brane web, this should be manifest in two inequivalent maximal subdivisions, as for instance in [24]. However, so far there is no sign of more than one maximal subdivision in any of the cases based on O5 planes. In contrast, the $\mathrm{O}^{-}$construction does admit two inequivalent subdivision such that the Higgs branch is, in fact, recovered to be a union of two cones.

In addition, for cases with non-complete Higgsing such as $\operatorname{Sp}(k)$ with $N_{f}<2 k$ flavours as well as $\mathrm{O}(k)$ with $N_{f} \leq k-3$, one can expect the appearance of nilpotent operators, as discussed for $\mathrm{SU}(k)$ gauge theories in [59]. Further progress in this direction requires, in part, a detailed analysis of the Higgs branch using different methods.

\section{Acknowledgments}

We are grateful to Santiago Cabrera, Hirotaka Hayashi, Rudolph Kalveks, Sung-Soo Kim, Kimyeong Lee, Dominik Miketa, Sakura Schäfer-Nameki, Futoshi Yagi, Gabi Zafrir, and Anton Zajac for helpful discussions. The work of A.B., J.F.G., A.H. and Z.Z. was supported by STFC grant ST/P000762/1. The work of M.S. was supported by the National Thousand-Young-Talents Program of China, the National Natural Science Foundation of China (grant no. 11950410497), and the China Postdoctoral Science Foundation (grant no. 2019M650616). We thank the Simons Center for Geometry and Physics, Stony Brook University for the hospitality and the partial support during an early stage of this work at the Simons Summer workshop 2019. We thank the 11th Joburg Workshop on String Theory from 8-13 December 2019 for hospitality, and the MIT-Imperial College London Seed Fund for support. 


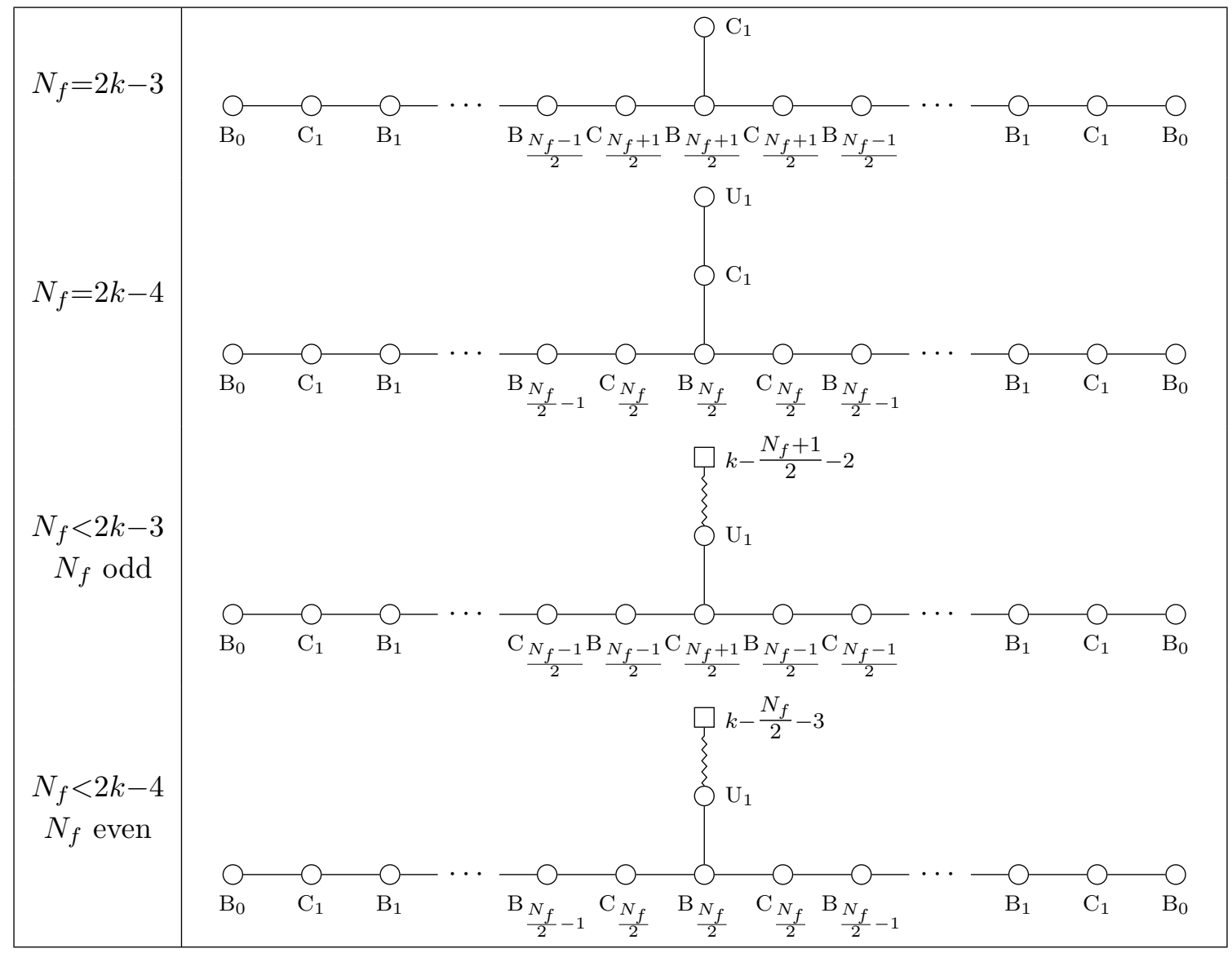

Table 11. Infinite coupling Higgs branches for $\mathrm{O}(2 k)$ theories with $N_{f}$ flavours. The moduli space dimensions correctly match the increase due to the new Higgs branch directions at infinite coupling [10] compared to finite coupling of table 9.

\section{A Background material}

\section{A.1 Type II brane configurations with 8 supercharges}

Consider Type II superstring theory with

- NS5 branes in $x^{0}, x^{1}, \ldots, x^{5}$ direction,

- $\mathrm{D} p$ branes in $x^{0}, x^{1}, \ldots, x^{p-1}, x^{6}$, direction,

- $\mathrm{D}(p+2)$ branes in $x^{0}, x^{1}, \ldots, x^{p-1}, x^{7}, x^{8}, x^{9}$ direction.

For $0 \leq p \leq 6$ the configuration preserves 8 supercharges and gives rise to a $p$ dimensional world-volume theory living on the $\mathrm{D} p$ branes suspended between NS5 branes. Since every such $\mathrm{D} p-\mathrm{D}(p+2)$-NS5 brane configuration is T-dual to the set-up of [62], a $\mathrm{D} p$ brane is created or annihilated whenever a NS5 passes through a $\mathrm{D}(p+2)$ brane. In the presence of $\mathrm{O} p$ planes, brane creation and annihilation is modified due to the non-trivial brane charge of the orientifold. In units of the physical $\mathrm{D} p$ branes, the RR-charges of the $\mathrm{O} p$ planes are as follows [87, 97]:

$$
\operatorname{charge}\left(\mathrm{O} p^{ \pm}\right)= \pm 2^{p-5}, \quad \operatorname{charge}\left(\widetilde{\mathrm{O} p}^{-}\right)=\frac{1}{2}-2^{p-5}, \quad \operatorname{charge}\left(\widetilde{\mathrm{O} p}^{+}\right)=2^{p-5}
$$




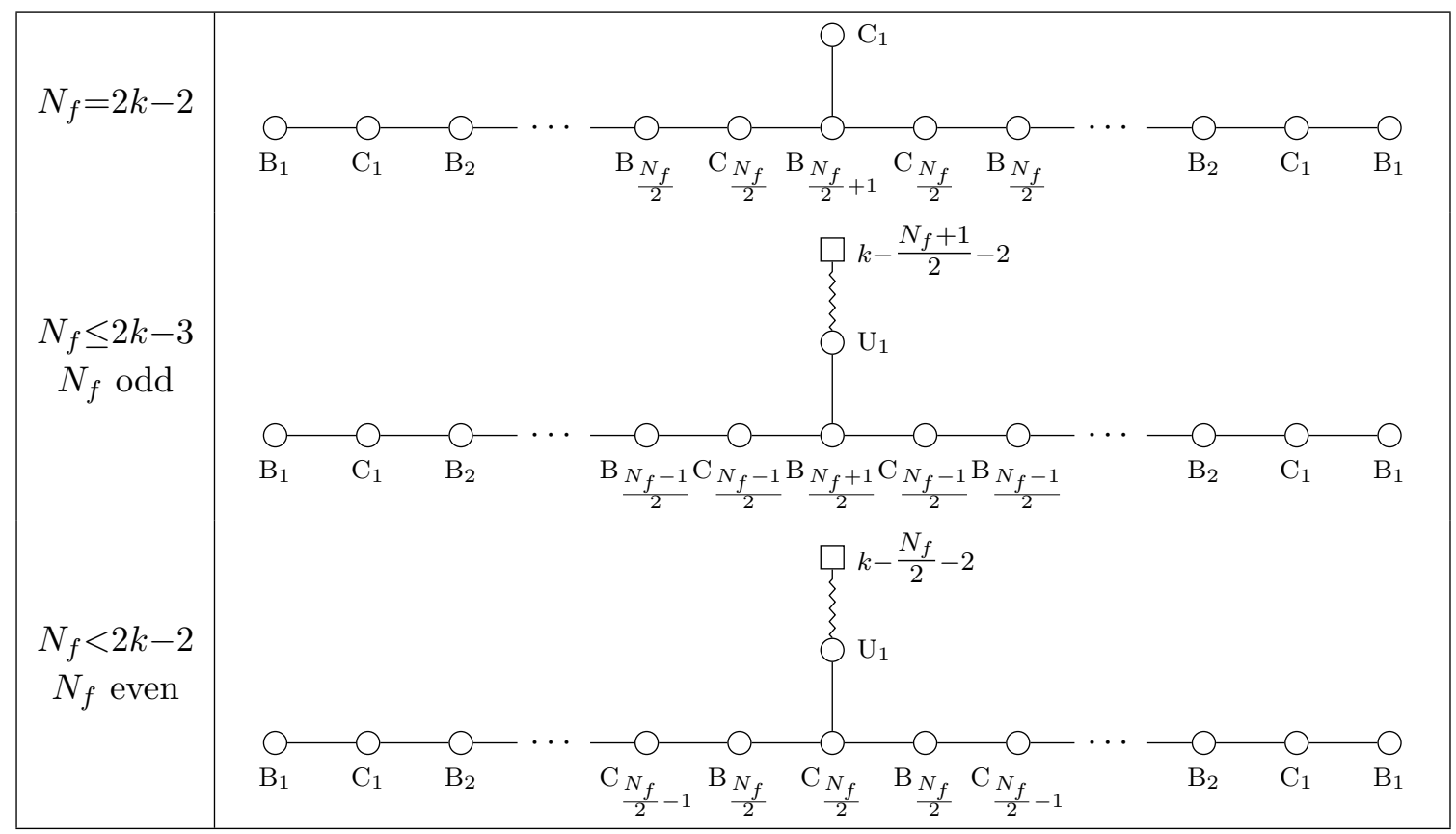

Table 12. Infinite coupling Higgs branches for $\mathrm{O}(2 k+1)$ theories with $N_{f}$ flavours. The moduli space dimensions match the increase due to the new Higgs branch directions at infinite coupling [10] compared to finite coupling of table 10 .

As a remark, the NS-charges of the $\mathrm{O} p$ planes are zero. Moreover, the orientifolds change whenever passing through a half NS5 or half $\mathrm{D}(p+2)$ brane [84, 89, 97]: on the one hand, an $\mathrm{O} p^{ \pm}$passing through a half NS5 turns into an $\mathrm{O} p^{\mp}$; likewise, an $\widetilde{\mathrm{O}}^{ \pm}$becomes an $\widetilde{\mathrm{O} p}^{\mp}$. On the other hand, an $\mathrm{O} p^{ \pm}$passing through a half $\mathrm{D}(p+2)$ turns into an $\widetilde{\mathrm{O} p}^{ \pm}$, and vice versa.

If there are $\mathrm{D} p$ branes parallel to the $\mathrm{O} p$ planes, the world volume gauge theory becomes ortho-symplectic. Similarly, effects are induced on the world volume of the $\mathrm{D}(p+2)$. Table 6 summarises these known results and introduces the notation, which follows [100].

\section{A.2 5-branes webs with 7-branes and orientifold 5-planes}

For this paper, $(p, q) 5$-branes webs in the presence of $[p, q] 7$-branes and orientifold 5 -planes are relevant. The integers $p$ and $q$ are assumed to be coprime throughout. The Type IIB brane construction is summarised in table 5 .

Brane bending due to orientifold planes Since O5 planes change whenever crossing an half NS5 brane, one finds that a $(0,1) 5$-brane (i.e. an NS5 brane) that separates a $\mathrm{O}^{+}$ and $\mathrm{O} 5^{-}$plane is bent. Using charge conservation, the quantum corrected configuration becomes [10]:

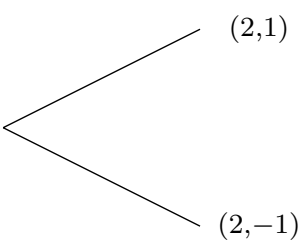


Attempting to repeat the analysis for a classical $(0,1) 5$-brane separating a $\widetilde{\mathrm{O}}^{+}$and $\widetilde{\mathrm{O}}^{-}$ plane is known to be troublesome due to arising fractional brane charges. A solution involving monodromy cuts has been proposed in [10].

Monodromy of the 7-brane. For the $[p, q]$ 7-brane, the associated monodromy matrix is given by

$$
M_{[p, q]}=\left(\begin{array}{cc}
1-p q & p^{2} \\
-q^{2} & 1+p q
\end{array}\right)
$$

such that the action is clockwise; in more detail, a schematic depiction looks like

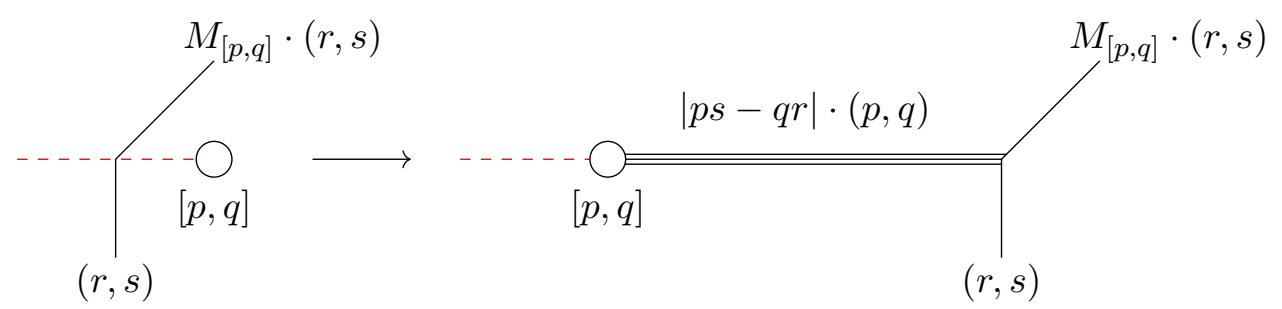

where $M_{[p, q]} \cdot(r, s)$ denotes the matrix product with a vector.

$\widetilde{\mathrm{O} 5}^{+}$and $\widetilde{\mathrm{O5}}^{-}$planes and bending. Following an argument presented in [10], one considers the well-defined set-up (A.2), adds two [1,0] 7-branes on the left-hand-side

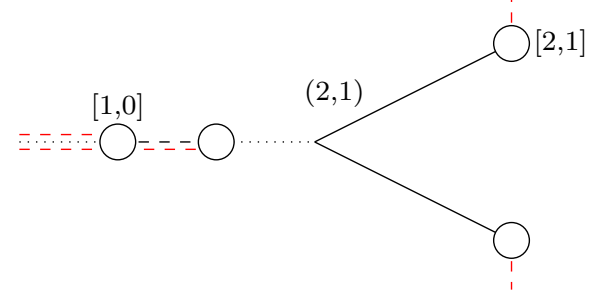

and moves them successively through the 5-branes, accounting for brane creation and annihilation. The first transitions is not accompanied by brane creation

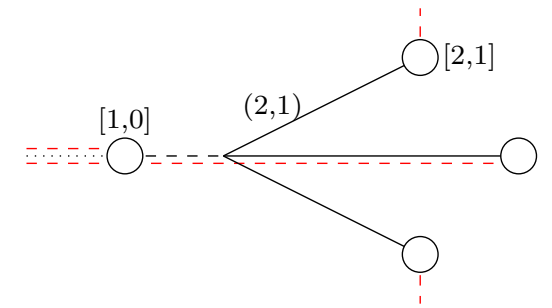

while the second transition is indeed accompanied by brane creation

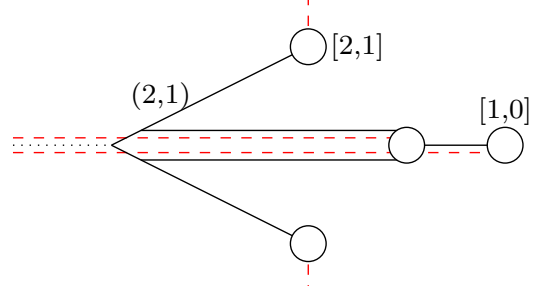


Thereafter, one can move the monodromy cuts, which then affects the $(2,1) 5$-brane as described in (A.3). Thus,

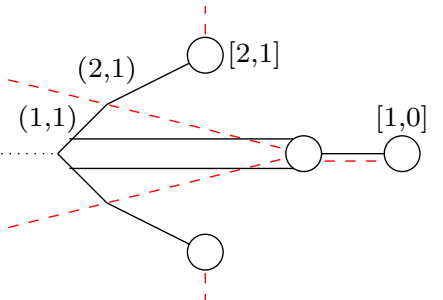

and finally one ends up with

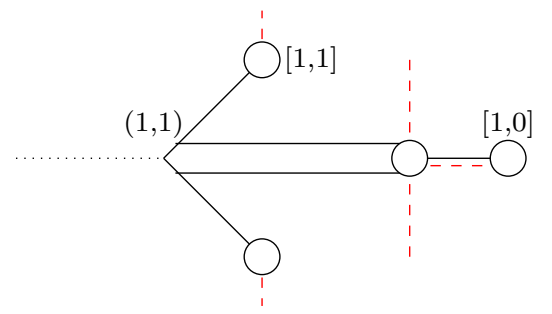

Hence, the understanding of $\widetilde{\mathrm{O}}^{+}$and $\widetilde{\mathrm{O}}^{-}$becomes

- $\widetilde{\mathrm{O} 5}^{-}$equals an $\mathrm{O}^{-}$together with half a stuck D5 and a (half-) monodromy cut.

- $\widetilde{\mathrm{O} 5}^{+}$contains a (half-) monodromy cut as well.

The (half-)monodromy cut is associated to a [1,0] 7-brane and coincides with the orientifold drawn $\left(x^{5}, x^{6}\right)$-plane.

Splitting 7-branes on O5 planes. Having half 7-branes merging on a O5 plane and subsequently splitting along the orientifold, one needs to take care of how branes are created in the process. The different cases are summarised in table 13.

\section{A.3 $3 \mathrm{~d} \mathcal{N}=4$ Coulomb branches}

Associated to a magnetic quiver is a space of dressed monopole operators or, loosely speaking, a $3 \mathrm{~d} \mathcal{N}=4$ Coulomb branch. In particular for linear quiver gauge theories of either unitary gauge groups or alternating ortho-symplectic gauge nodes, the IR global symmetry $G$ is usually well approximated by analysing the subset of balanced nodes.

Unitary quivers. A unitary gauge node $\mathrm{U}(k)$ with $N_{f}$ flavours is called [100, section 2.4]

$$
\text { good if } \quad N_{f} \geq 2 k, \quad \text { and balanced if } \quad N_{f}=2 k .
$$

Then for a quiver comprised of unitary nodes the following is expected to hold:

- The subset of balanced gauge nodes forms the Dynkin diagram of the non-abelian part of the Coulomb branch global symmetry.

- The number of unbalanced nodes minus one yields the number of U(1) factors inside the Coulomb branch global symmetry.

Note that this procedure might only provide a subgroup of the global symmetry of the Coulomb branch. 


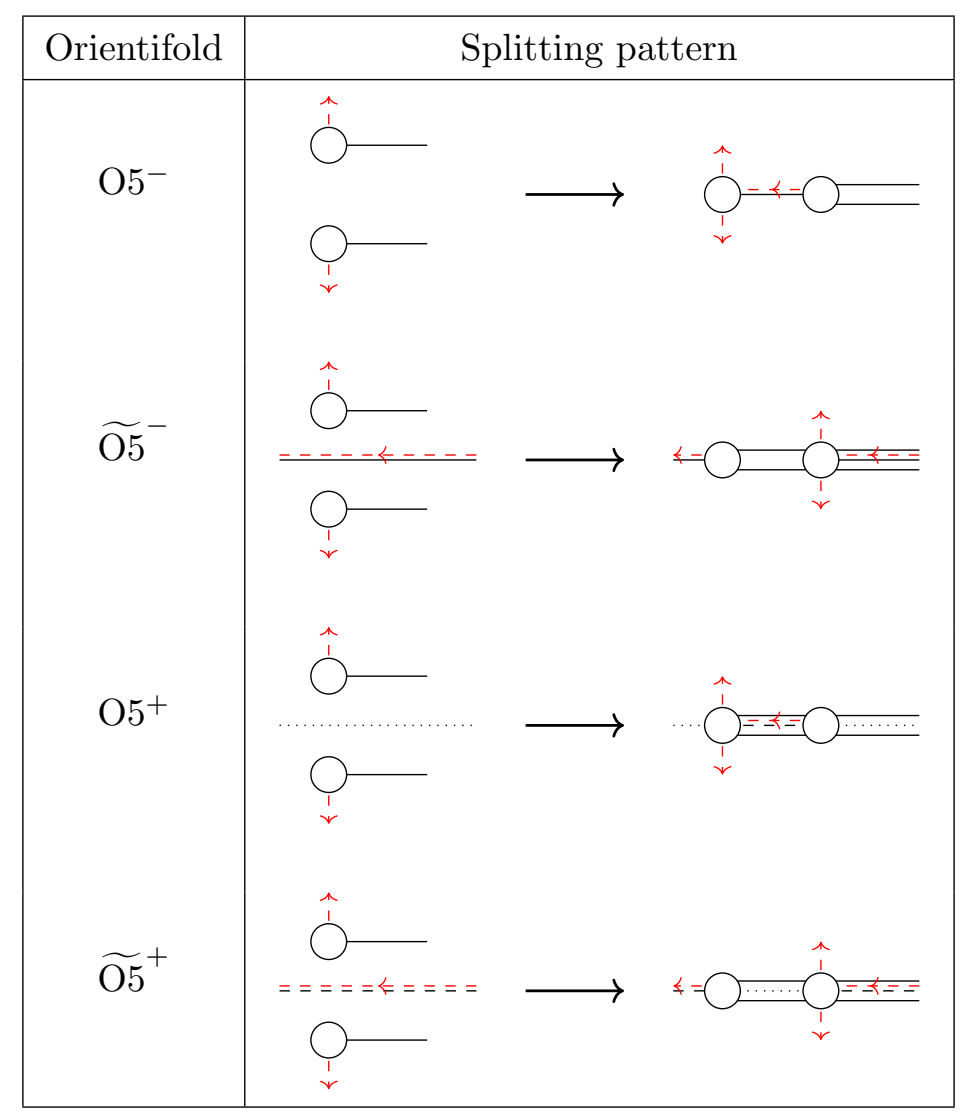

Table 13. Splitting of D7 branes, with D5 branes ending on them, on various O5 planes.

Ortho-symplectic linear quivers. For orthogonal and symplectic $3 \mathrm{~d} \mathcal{N}=4$ gauge theories, the conditions for good and balanced are as follows [100, section 5.1-5.2]: a $\mathrm{O}(k)$ (or $\mathrm{SO}(k)$ ) gauge group with fundamental flavours, transforming under a $\operatorname{USp}\left(2 N_{f}\right)$ global symmetry, is called

$$
\text { good if } \quad N_{f} \geq k-1, \quad \text { and balanced if } \quad N_{f}=k-1 .
$$

Similarly, a $\operatorname{USp}(2 k)=\operatorname{Sp}(k)$ gauge group with fundamental flavours, transforming under a $\mathrm{O}\left(2 N_{f}\right)$ global symmetry, is called

$$
\text { good if } \quad N_{f} \geq 2 k+1, \quad \text { and balanced if } \quad N_{f}=2 k+1 .
$$

Consider a linear quiver with alternating orthogonal and symplectic gauge nodes, then a chain of $p$ balanced nodes is expected to give rise to the following enhanced Coulomb branch symmetry $G$ :

- An $\mathrm{SO}(p+1)$ symmetry, if there are no $\mathrm{SO}(2)$ (or $\mathrm{O}(2)$ ) gauge nodes at the ends.

- An $\mathrm{SO}(p+2)$ symmetry, if there is an $\mathrm{SO}(2)$ (or $\mathrm{O}(2)$ ) gauge node at one of the ends.

- An $\mathrm{SO}(p+3)$ symmetry, if there is an $\mathrm{SO}(2)$ (or $\mathrm{O}(2)$ ) at each end.

\section{A.4 Summary companion paper}

As indicated in the main text, the choice of gauge group for a unitary-orthosymplectic quiver needs to be specified when considering the Coulomb branch moduli space. In this 
appendix, the main idea of the companion paper [80] is summarised. Based on the brane configurations, the magnetic gauge nodes are associated with the following groups

$$
\mathrm{B}_{n} \rightarrow \mathrm{SO}(2 n+1), \quad \mathrm{C}_{n} \rightarrow \mathrm{USp}(2 n), \quad \mathrm{D}_{n} \rightarrow \mathrm{SO}(2 n) .
$$

However, the choice of overall magnetic gauge group of a given quiver remains and is resolved in [80] as follows:

- Framed orthosymplectic quiver: the magnetic gauge group of the quiver is the product group over all nodes, because the kernel of the representation defining the matter content is trivial.

- Unframed (unitary-) orthosymplectic quiver without any $\mathrm{SO}$ (odd): the matter representation for the product gauge group has a nontrivial $\mathbb{Z}_{2}$ kernel. In order to reproduce the expected moduli spaces, the gauge group is chosen to be the product gauge group modulo this diagonal $\mathbb{Z}_{2}$.

- Unframed (unitary-) orthosymplectic quiver with $\mathrm{SO}$ (odd): the matter representation for the product gauge group has a trivial kernel. Hence, the gauge group of the quiver is the product gauge group.

Once the gauge group of a quiver is specified, the magnetic lattice is uniquely determined and the Coulomb branch Hilbert series can be evaluated via [63].

Open Access. This article is distributed under the terms of the Creative Commons Attribution License (CC-BY 4.0), which permits any use, distribution and reproduction in any medium, provided the original author(s) and source are credited.

\section{References}

[1] N. Seiberg, Five-dimensional SUSY field theories, nontrivial fixed points and string dynamics, Phys. Lett. B 388 (1996) 753 [hep-th/9608111] [INSPIRE].

[2] D.R. Morrison and N. Seiberg, Extremal transitions and five-dimensional supersymmetric field theories, Nucl. Phys. B 483 (1997) 229 [hep-th/9609070] [INSPIRE].

[3] K.A. Intriligator, D.R. Morrison and N. Seiberg, Five-dimensional supersymmetric gauge theories and degenerations of Calabi-Yau spaces, Nucl. Phys. B 497 (1997) 56 [hep-th/9702198] [INSPIRE].

[4] O. Aharony and A. Hanany, Branes, superpotentials and superconformal fixed points, Nucl. Phys. B 504 (1997) 239 [hep-th/9704170] [INSPIRE].

[5] O. Aharony, A. Hanany and B. Kol, Webs of $(p, q)$ five-branes, five-dimensional field theories and grid diagrams, JHEP 01 (1998) 002 [hep-th/9710116] [INSPIRE].

[6] O. DeWolfe, A. Hanany, A. Iqbal and E. Katz, Five-branes, seven-branes and five-dimensional $E_{n}$ field theories, JHEP 03 (1999) 006 [hep-th/9902179] [INSPIRE].

[7] M.R. Douglas, S.H. Katz and C. Vafa, Small instantons, Del Pezzo surfaces and type-I-prime theory, Nucl. Phys. B 497 (1997) 155 [hep-th/9609071] [INSPIRE].

[8] I. Brunner and A. Karch, Branes and six-dimensional fixed points, Phys. Lett. B 409 (1997) 109 [hep-th/9705022] [INSPIRE]. 
[9] O. Bergman and G. Zafrir, 5d fixed points from brane webs and O7-planes, JHEP 12 (2015) 163 [arXiv: 1507.03860] [INSPIRE].

[10] G. Zafrir, Brane webs and O5-planes, JHEP 03 (2016) 109 [arXiv:1512.08114] [INSPIRE].

[11] H. Hayashi, S.-S. Kim, K. Lee, M. Taki and F. Yagi, More on $5 d$ descriptions of $6 d$ SCFTs, JHEP 10 (2016) 126 [arXiv:1512.08239] [INSPIRE].

[12] L. Bao, E. Pomoni, M. Taki and F. Yagi, M5-Branes, Toric Diagrams and Gauge Theory Duality, JHEP 04 (2012) 105 [arXiv:1112.5228] [INSPIRE].

[13] O. Bergman and G. Zafrir, Lifting 4d dualities to 5d, JHEP 04 (2015) 141 [arXiv:1410.2806] [INSPIRE].

[14] S.-S. Kim, M. Taki and F. Yagi, Tao Probing the End of the World, PTEP 2015 (2015) 083B02 [arXiv: 1504.03672] [INSPIRE].

[15] H. Hayashi, S.-S. Kim, K. Lee, M. Taki and F. Yagi, A new $5 d$ description of $6 d$ D-type minimal conformal matter, JHEP 08 (2015) 097 [arXiv: 1505.04439] [INSPIRE].

[16] D. Gaiotto and H.-C. Kim, Duality walls and defects in $5 d \mathcal{N}=1$ theories, JHEP 01 (2017) 019 [arXiv: 1506.03871] [INSPIRE].

[17] G. Zafrir, Brane webs, 5d gauge theories and $6 d \mathcal{N}=(1,0)$ SCFT's, JHEP 12 (2015) 157 [arXiv: 1509.02016] [INSPIRE].

[18] H. Hayashi, S.-S. Kim, K. Lee and F. Yagi, 6d SCFTs, 5d Dualities and Tao Web Diagrams, JHEP 05 (2019) 203 [arXiv: 1509.03300] [INSPIRE].

[19] K. Ohmori and H. Shimizu, $S^{1} / T^{2}$ compactifications of $6 d \mathcal{N}=(1,0)$ theories and brane webs, JHEP 03 (2016) 024 [arXiv: 1509.03195] [INSPIRE].

[20] G. Zafrir, Brane webs in the presence of an $\mathrm{O5}^{-}$-plane and $4 d$ class $S$ theories of type $D$, JHEP 07 (2016) 035 [arXiv:1602.00130] [INSPIRE].

[21] H. Hayashi, S.-S. Kim, K. Lee and F. Yagi, Equivalence of several descriptions for $6 d$ SCFT, JHEP 01 (2017) 093 [arXiv: 1607.07786] [INSPIRE].

[22] H. Hayashi, S.-S. Kim, K. Lee and F. Yagi, Discrete theta angle from an O5-plane, JHEP 11 (2017) 041 [arXiv: 1707.07181] [INSPIRE].

[23] H. Hayashi, S.-S. Kim, K. Lee and F. Yagi, Dualities and 5-brane webs for $5 d$ rank 2 SCFTs, JHEP 12 (2018) 016 [arXiv:1806.10569] [INSPIRE].

[24] S. Cabrera, A. Hanany and F. Yagi, Tropical Geometry and Five Dimensional Higgs Branches at Infinite Coupling, JHEP 01 (2019) 068 [arXiv:1810.01379] [INSPIRE].

[25] H. Hayashi, S.-S. Kim, K. Lee and F. Yagi, Rank-3 antisymmetric matter on 5-brane webs, JHEP 05 (2019) 133 [arXiv:1902.04754] [INSPIRE].

[26] H. Hayashi, S.-S. Kim, K. Lee and F. Yagi, Complete prepotential for $5 d \mathcal{N}=1$ superconformal field theories, JHEP 02 (2020) 074 [arXiv: 1912.10301] [INSPIRE].

[27] H.-C. Kim, S.-S. Kim and K. Lee, 5-dim Superconformal Index with Enhanced En Global Symmetry, JHEP 10 (2012) 142 [arXiv:1206.6781] [INSPIRE].

[28] D. Rodríguez-Gómez and G. Zafrir, On the $5 d$ instanton index as a Hilbert series, Nucl. Phys. B 878 (2014) 1 [arXiv:1305.5684] [InSPIRE].

[29] O. Bergman, D. Rodríguez-Gómez and G. Zafrir, $5 d$ superconformal indices at large $N$ and holography, JHEP 08 (2013) 081 [arXiv:1305.6870] [INSPIRE]. 
[30] O. Bergman, D. Rodríguez-Gómez and G. Zafrir, Discrete $\theta$ and the $5 d$ superconformal index, JHEP 01 (2014) 079 [arXiv:1310.2150] [INSPIRE].

[31] M. Taki, Notes on Enhancement of Flavor Symmetry and 5d Superconformal Index, arXiv: 1310.7509 [INSPIRE].

[32] O. Bergman, D. Rodríguez-Gómez and G. Zafrir, 5-Brane Webs, Symmetry Enhancement, and Duality in 5d Supersymmetric Gauge Theory, JHEP 03 (2014) 112 [arXiv:1311.4199] [INSPIRE].

[33] C. Hwang, J. Kim, S. Kim and J. Park, General instanton counting and 5d SCFT, JHEP 07 (2015) 063 [Addendum ibid. 04 (2016) 094] [arXiv: 1406.6793] [INSPIRE].

[34] G. Zafrir, Duality and enhancement of symmetry in 5d gauge theories, JHEP 12 (2014) 116 [arXiv: 1408.4040] [INSPIRE].

[35] G. Zafrir, Instanton operators and symmetry enhancement in 5d supersymmetric USp, SO and exceptional gauge theories, JHEP 07 (2015) 087 [arXiv:1503.08136] [INSPIRE].

[36] K. Yonekura, Instanton operators and symmetry enhancement in 5d supersymmetric quiver gauge theories, JHEP 07 (2015) 167 [arXiv:1505.04743] [INSPIRE].

[37] D. Bashkirov, A comment on the enhancement of global symmetries in superconformal $\mathrm{SU}(2)$ gauge theories in 5D, arXiv:1211.4886 [INSPIRE].

[38] A. Iqbal and C. Vafa, BPS Degeneracies and Superconformal Index in Diverse Dimensions, Phys. Rev. D 90 (2014) 105031 [arXiv:1210.3605] [InSPIRE].

[39] L. Bao, V. Mitev, E. Pomoni, M. Taki and F. Yagi, Non-Lagrangian Theories from Brane Junctions, JHEP 01 (2014) 175 [arXiv:1310.3841] [INSPIRE].

[40] H. Hayashi, H.-C. Kim and T. Nishinaka, Topological strings and $5 d T_{N}$ partition functions, JHEP 06 (2014) 014 [arXiv:1310.3854] [INSPIRE].

[41] H. Hayashi and G. Zoccarato, Exact partition functions of Higgsed $5 d T_{N}$ theories, JHEP 01 (2015) 093 [arXiv: 1409.0571] [INSPIRE].

[42] V. Mitev, E. Pomoni, M. Taki and F. Yagi, Fiber-Base Duality and Global Symmetry Enhancement, JHEP 04 (2015) 052 [arXiv: 1411.2450] [INSPIRE].

[43] S.-S. Kim and F. Yagi, 5d E $E_{n}$ Seiberg-Witten curve via toric-like diagram, JHEP 06 (2015) 082 [arXiv: 1411.7903] [INSPIRE].

[44] H. Hayashi and G. Zoccarato, Topological vertex for Higgsed $5 d T_{N}$ theories, JHEP 09 (2015) 023 [arXiv:1505.00260] [INSPIRE].

[45] N. Lambert, C. Papageorgakis and M. Schmidt-Sommerfeld, Instanton Operators in Five-Dimensional Gauge Theories, JHEP 03 (2015) 019 [arXiv:1412.2789] [InSPIRE].

[46] D. Rodriguez-Gomez and J. Schmude, Supersymmetrizing 5d instanton operators, JHEP 03 (2015) 114 [arXiv: 1501.00927] [inSPIRE].

[47] Y. Tachikawa, Instanton operators and symmetry enhancement in 5d supersymmetric gauge theories, PTEP 2015 (2015) 043B06 [arXiv:1501.01031] [INSPIRE].

[48] F. Apruzzi, C. Lawrie, L. Lin, S. Schäfer-Nameki and Y.-N. Wang, 5d Superconformal Field Theories and Graphs, Phys. Lett. B 800 (2020) 135077 [arXiv:1906.11820] [InSPIRE].

[49] F. Apruzzi, C. Lawrie, L. Lin, S. Schäfer-Nameki and Y.-N. Wang, Fibers add Flavor, Part I: Classification of 5d SCFTs, Flavor Symmetries and BPS States, JHEP 11 (2019) 068 [arXiv: 1907.05404] [INSPIRE]. 
[50] F. Apruzzi, C. Lawrie, L. Lin, S. Schäfer-Nameki and Y.-N. Wang, Fibers add Flavor, Part II: 5d SCFTs, Gauge Theories, and Dualities, JHEP 03 (2020) 052 [arXiv:1909.09128] [INSPIRE].

[51] F. Apruzzi, S. Schäfer-Nameki and Y.-N. Wang, 5d SCFTs from Decoupling and Gluing, arXiv: 1912.04264 [INSPIRE].

[52] P. Jefferson, H.-C. Kim, C. Vafa and G. Zafrir, Towards Classification of 5d SCFTs: Single Gauge Node, arXiv:1705.05836 [INSPIRE].

[53] P. Jefferson, S. Katz, H.-C. Kim and C. Vafa, On Geometric Classification of 5d SCFTs, JHEP 04 (2018) 103 [arXiv: 1801.04036] [INSPIRE].

[54] L. Bhardwaj, On the classification of $5 d$ SCFTs, arXiv:1909.09635 [INSPIRE].

[55] L. Bhardwaj and G. Zafrir, Classification of $5 d N=1$ gauge theories, arXiv:2003.04333 [INSPIRE].

[56] N.J. Hitchin, A. Karlhede, U. Lindström and M. Roček, HyperKähler Metrics and Supersymmetry, Commun. Math. Phys. 108 (1987) 535 [INSPIRE].

[57] S. Cremonesi, G. Ferlito, A. Hanany and N. Mekareeya, Instanton Operators and the Higgs Branch at Infinite Coupling, JHEP 04 (2017) 042 [arXiv:1505.06302] [INSPIRE].

[58] G. Ferlito, A. Hanany, N. Mekareeya and G. Zafrir, $3 d$ Coulomb branch and $5 d$ Higgs branch at infinite coupling, JHEP 07 (2018) 061 [arXiv:1712.06604] [INSPIRE].

[59] A. Bourget, S. Cabrera, J.F. Grimminger, A. Hanany and Z. Zhong, Brane Webs and Magnetic Quivers for SQCD, JHEP 03 (2020) 176 [arXiv:1909.00667] [INSPIRE].

[60] S. Cabrera, A. Hanany and M. Sperling, Magnetic quivers, Higgs branches, and $6 d$ $N=(1,0)$ theories, JHEP 06 (2019) 071 [Erratum ibid. 07 (2019) 137] [arXiv: 1904.12293] [INSPIRE].

[61] S. Cabrera, A. Hanany and M. Sperling, Magnetic quivers, Higgs branches, and $6 \mathrm{~d}$ $\mathcal{N}=(1,0)$ theories - orthogonal and symplectic gauge groups, JHEP 02 (2020) 184 [arXiv: 1912. 02773] [INSPIRE].

[62] A. Hanany and E. Witten, Type IIB superstrings, BPS monopoles, and three-dimensional gauge dynamics, Nucl. Phys. B 492 (1997) 152 [hep-th/9611230] [InSPIRE].

[63] S. Cremonesi, A. Hanany and A. Zaffaroni, Monopole operators and Hilbert series of Coulomb branches of $3 d \mathcal{N}=4$ gauge theories, JHEP 01 (2014) 005 [arXiv:1309.2657] [INSPIRE].

[64] N. Mekareeya, K. Ohmori, Y. Tachikawa and G. Zafrir, $E_{8}$ instantons on type-A ALE spaces and supersymmetric field theories, JHEP 09 (2017) 144 [arXiv:1707.04370] [INSPIRE].

[65] A. Hanany and N. Mekareeya, The small E $E_{8}$ instanton and the Kraft Procesi transition, JHEP 07 (2018) 098 [arXiv:1801.01129] [INSPIRE].

[66] A. Hanany and G. Zafrir, Discrete Gauging in Six Dimensions, JHEP 07 (2018) 168 [arXiv: 1804.08857] [INSPIRE].

[67] M. Del Zotto and A. Hanany, Complete Graphs, Hilbert Series, and the Higgs branch of the $4 d \mathcal{N}=2\left(A_{n}, A_{m}\right) S C F T s$, Nucl. Phys. B 894 (2015) 439 [arXiv:1403.6523] [INSPIRE].

[68] H. Kraft and C. Procesi, Minimal singularities in $G L_{n}$, Invent. Math. 62 (1980) 503.

[69] H. Kraft and C. Procesi, On the geometry of conjugacy classes in classical groups, Comment. Math. Helv. 57 (1982) 539. 
[70] A. Bourget et al., The Higgs mechanism - Hasse diagrams for symplectic singularities, JHEP 01 (2020) 157 [arXiv: 1908. 04245] [INSPIRE].

[71] A. Beauville, Symplectic singularities, Invent. Math. 139 (2000) 541 [math/9903070].

[72] S. Cabrera and A. Hanany, Branes and the Kraft-Procesi Transition, JHEP 11 (2016) 175 [arXiv: 1609.07798] [INSPIRE].

[73] S. Cabrera and A. Hanany, Branes and the Kraft-Procesi transition: classical case, JHEP 04 (2018) 127 [arXiv: 1711.02378] [INSPIRE].

[74] S. Cabrera and A. Hanany, Quiver Subtractions, JHEP 09 (2018) 008 [arXiv:1803.11205] [INSPIRE].

[75] M. Bullimore, T. Dimofte and D. Gaiotto, The Coulomb Branch of $3 d \mathcal{N}=4$ Theories, Commun. Math. Phys. 354 (2017) 671 [arXiv: 1503.04817] [INSPIRE].

[76] H. Nakajima, Towards a mathematical definition of Coulomb branches of 3-dimensional $\mathcal{N}=4$ gauge theories, I, Adv. Theor. Math. Phys. 20 (2016) 595 [arXiv:1503.03676] [INSPIRE].

[77] A. Braverman, M. Finkelberg and H. Nakajima, Towards a mathematical definition of Coulomb branches of 3-dimensional $\mathcal{N}=4$ gauge theories, II, Adv. Theor. Math. Phys. 22 (2018) 1071 [arXiv: 1601.03586] [INSPIRE].

[78] O. Chacaltana and J. Distler, Tinkertoys for the $D_{N}$ series, JHEP 02 (2013) 110 [arXiv: 1106.5410] [INSPIRE].

[79] O. Chacaltana, J. Distler and Y. Tachikawa, Gaiotto duality for the twisted $A_{2 N-1}$ series, JHEP 05 (2015) 075 [arXiv:1212.3952] [INSPIRE].

[80] A. Bourget, J.F. Grimminger, A. Hanany, R. Kalveks, M. Sperling and Z. Zhong, Magnetic Lattices for Orthosymplectic Quivers, arXiv:2007.04667 [INSPIRE].

[81] A. Hanany and R. Kalveks, Quiver Theories and Hilbert Series of Classical Slodowy Intersections, Nucl. Phys. B 952 (2020) 114939 [arXiv:1909.12793] [INSPIRE].

[82] A. Hanany and R. Kalveks, Highest Weight Generating Functions for Hilbert Series, JHEP 10 (2014) 152 [arXiv: 1408.4690] [INSPIRE].

[83] A. Hanany and Z. Zhong, Highest-Weight Generating Functions for Tropical Quivers, to appear.

[84] N.J. Evans, C.V. Johnson and A.D. Shapere, Orientifolds, branes, and duality of 4-D gauge theories, Nucl. Phys. B 505 (1997) 251 [hep-th/9703210] [INSPIRE].

[85] K. Landsteiner, E. Lopez and D.A. Lowe, $N=2$ supersymmetric gauge theories, branes and orientifolds, Nucl. Phys. B 507 (1997) 197 [hep-th/9705199] [InSPIRE].

[86] F. Benini, S. Benvenuti and Y. Tachikawa, Webs of five-branes and $N=2$ superconformal field theories, JHEP 09 (2009) 052 [arXiv:0906.0359] [INSPIRE].

[87] B. Feng and A. Hanany, Mirror symmetry by O3 planes, JHEP 11 (2000) 033 [hep-th/0004092] [INSPIRE].

[88] A. Sen, Duality and orbifolds, Nucl. Phys. B 474 (1996) 361 [hep-th/9604070] [InSPIRE].

[89] A. Hanany and B. Kol, On orientifolds, discrete torsion, branes and M-theory, JHEP 06 (2000) 013 [hep-th/0003025] [INSPIRE].

[90] F. Benini, Y. Tachikawa and D. Xie, Mirrors of 3d Sicilian theories, JHEP 09 (2010) 063 [arXiv: 1007.0992] [InSPIRE]. 
[91] O. Chacaltana, J. Distler and Y. Tachikawa, Nilpotent orbits and codimension-two defects of $6 d N=(2,0)$ theories, Int. J. Mod. Phys. A 28 (2013) 1340006 [arXiv:1203.2930] [INSPIRE].

[92] S. Cabrera, A. Hanany and Z. Zhong, Nilpotent orbits and the Coulomb branch of $T^{\sigma}(G)$ theories: special orthogonal vs orthogonal gauge group factors, JHEP 11 (2017) 079 [arXiv: 1707.06941] [INSPIRE].

[93] Z. Zhong, Quiver gauge theories in 3d, 5d and 6d, MSc Thesis, Imperial College, London (2018).

[94] G. Ferlito and A. Hanany, A tale of two cones: the Higgs Branch of $\operatorname{Sp}(n)$ theories with $2 n$ flavours, arXiv:1609.06724 [INSPIRE].

[95] D.H. Collingwood and W.M. McGovern, Nilpotent orbits in semisimple Lie algebra: an introduction, CRC Press (1993).

[96] A. Sen, F theory and orientifolds, Nucl. Phys. B 475 (1996) 562 [hep-th/9605150] [INSPIRE].

[97] A. Hanany and A. Zaffaroni, Issues on orientifolds: On the brane construction of gauge theories with $\mathrm{SO}(2 N)$ global symmetry, JHEP 07 (1999) 009 [hep-th/9903242] [INSPIRE].

[98] A. Hanany and M. Sperling, Discrete quotients of 3-dimensional $\mathcal{N}=4$ Coulomb branches via the cycle index, JHEP 08 (2018) 157 [arXiv:1807.02784] [INSPIRE].

[99] A. Hanany and A. Zajac, Discrete Gauging in Coulomb branches of Three Dimensional $\mathcal{N}=4$ Supersymmetric Gauge Theories, JHEP 08 (2018) 158 [arXiv:1807.03221] [INSPIRE].

[100] D. Gaiotto and E. Witten, S-duality of Boundary Conditions In $N=4$ Super Yang-Mills Theory, Adv. Theor. Math. Phys. 13 (2009) 721 [arXiv:0807.3720] [InSPIRE]. 